\title{
Chiral Phosphoric Acid Catalyzed Asymmetric Oxidative Dearomatization of Naphthols with Quinones
}

Gongming Zhu, ${ }^{\ddagger \neq}$ Guangjun Bao, ${ }^{\ddagger}$ Yiping Li, ${ }^{\ddagger}$ Junxian Yang, ${ }^{\ddagger}$ Wangsheng Sun, ${ }^{* \neq}$ Jing $\mathrm{Li}^{, *+\ldots}$ Liang Hong ${ }^{*,+}$ and Rui Wang*,**

$\dagger$ School of Pharmaceutical Sciences, Sun Yat-sen University, Guangzhou 510006, China

¥Key Laboratory of Preclinical Study for New Drugs of Gansu Province, Lanzhou University, Lanzhou, 730000, China

E-mail: sunws@1zu.edu.cn; hongliang@sysu.edu.cn; wangrui@lzu.edu.cn 


\section{Table of Contents}

$\begin{array}{ll}\text { 1. General information } & \text { S2 }\end{array}$

2. Optimization of the reaction $\quad$ S3

3. General procedure for preparation of racemic $\quad$ S4

4.General experimental procedure for dearomatization of 2-naphthols with quinone $\quad$ S4

5. Further Transformations of the Products $\quad$ S5

$\begin{array}{ll}\text { 6. Characterization of } \mathbf{4} \text { and } 5 & \text { S6-17 }\end{array}$

$\begin{array}{ll}\text { 7. Determination of the absolute configuration } 4 & \text { S18-19 }\end{array}$

$\begin{array}{lr}\text { 8. HPLC analytic conditions of } \mathbf{4} \text { and } \mathbf{5} & \text { S20-23 }\end{array}$

9. Copies of HPLC spectra for $\mathbf{4}$ and 5

$\begin{array}{lr}\text { 10. Copies of NMR spectra for } 4 \text { and } 5 & \text { S44-85 }\end{array}$

\section{General information}

Unless stated otherwise, all reactions were carried out in flame dried glassware. All solvents were purified and dried according to standard methods prior to use. 2-naphthols ${ }^{1}$ and Cat. $3^{2}$ were prepared according to literature, quinones were purchased from commercial suppliers. ${ }^{1} \mathrm{H}$ and ${ }^{13} \mathrm{C}$ NMR spectra were recorded on a Varian instrument (300 MHz and $75 \mathrm{MHz}$, respectively) and internally referenced to tetramethylsilane signal or residual protio solvent signals. Data for ${ }^{1} \mathrm{H}$ NMR are recorded as follows: chemical shift $(\delta, \mathrm{ppm})$, multiplicity $(\mathrm{s}=$ singlet, $\mathrm{d}=$ doublet, $\mathrm{t}=$ triplet, $\mathrm{m}=$ multiplet, $\mathrm{q}=$ quartet or unresolved, coupling constant(s) in Hz, integration). Data for

${ }^{13} \mathrm{C}$ NMR are reported in terms of chemical shift $(\delta, \mathrm{ppm})$. IR spectra were recorded on a FT-IR spectrometer and only major peaks were reported in $\mathrm{cm}^{-1}$. Optical rotations were reported as follows: $[\alpha]_{\mathrm{D}}{ }^{\mathrm{rt}}$ (c: $\mathrm{g} / 100 \mathrm{~mL}$, in solvent). High resolution mass spectra (HRMS) were obtained by the ESI ionization sources. The ee value determination was carried out using chiral HPLC with Daicel Chiracel column on Waters with a 996 UV-detector.

[1] (a) A. Rudolph, P. H. Bos, A. Meetsma, A. J. Minnaard, B. L. Feringa, Angew. Chem. Int. Ed. 2011, 50, 5834 -5838. (b) T. Oguma, T. Katsuki, J. Am. Chem. Soc., 2012, 134, 20017-20020. (c) J. Zheng, S.-B. Wang, C. Zheng, S.-L. You, J. Am. Chem. Soc., 2015, 137, 4880-4883.

[2] (a) S. Müller, M. J. Webber, B. List, J. Am. Chem. Soc., 2011, 133, 18534-18537. (b) I. Čorić, S. Müller, B. List, J. Am. Chem. Soc., 2010, 132, 17370-17373. (c) I. T. Crouch, R. K. Neff, D. E. Frantz, J. Am. Chem. Soc., 2013, 135, 4970-4973. (d) F. R-Michailidis, L. Guenee, A, Alexakis, Angew. Chem. Int. Ed. 2013, 52, 9266-9270. (e) M. Yamanaka, J. Itoh, K. Fuchibe, T. Akiyama, J. Am. Chem. Soc. 2007, 129, 6756-6764 
2. Optimization of the reaction condition

\begin{tabular}{|c|c|c|c|c|c|c|c|}
\hline entry & cat. & solvent & 2a:1a & & time (h) & conv. $(\%)$ & ee $(\%)$ \\
\hline 1 & $\mathbf{3 a}$ & toluene & $1: 3$ & air & 48 & 45 & 69 \\
\hline 2 & $3 b$ & toluene & $1: 3$ & air & 48 & $>95$ & 42 \\
\hline 3 & $3 c$ & toluene & $1: 3$ & air & 48 & $>95$ & 35 \\
\hline 4 & $3 d$ & toluene & $1: 3$ & air & 48 & $>95(77)$ & 75 \\
\hline 5 & $3 e$ & toluene & $1: 3$ & air & 48 & $>95$ & 23 \\
\hline 6 & $3 f$ & toluene & $1: 3$ & air & 48 & 30 & 24 \\
\hline 7 & $3 g$ & toluene & $1: 3$ & air & 48 & 89 & 91 \\
\hline 8 & $3 g$ & DCM & $1: 3$ & air & 36 & $>95(78)$ & 98 \\
\hline 9 & $3 g$ & DCE & $1: 3$ & air & 36 & $>95$ & 97 \\
\hline 10 & $3 g$ & xylene & $1: 3$ & air & 48 & $>95$ & 89 \\
\hline 11 & $3 g$ & mesitylene & $1: 3$ & air & 48 & $>95$ & 86 \\
\hline 12 & $3 g$ & $\mathrm{PhF}$ & $1: 3$ & air & 48 & $>95$ & 93 \\
\hline 13 & $3 g$ & THF & $1: 3$ & air & 48 & $<5$ & -- \\
\hline 14 & $3 g$ & $\mathrm{Et}_{2} \mathrm{O}$ & $1: 3$ & air & 48 & $<5$ & -- \\
\hline 15 & $3 g$ & DCM & $1: 4$ & air & 36 & $>95(76)$ & 97 \\
\hline 16 & $3 g$ & DCM & $1: 6$ & air & 36 & $>95(68)$ & 97 \\
\hline 17 & $3 g$ & DCM & $1: 2$ & air & 36 & $76(53)$ & 98 \\
\hline 18 & $3 g$ & DCM & $1: 1$ & air & 36 & $45(28)$ & 98 \\
\hline 19 & $3 g$ & DCM & $1: 3$ & $A_{r}$ & 36 & $>95(77)$ & 98 \\
\hline 20 & $3 g$ & $\mathrm{DCM}$ & $1: 3$ & $\mathrm{O}_{2}$ & 36 & $>95(75)$ & 98 \\
\hline 21 & $3 g$ & DCM & $1: 3$ & DDQ & 48 & $>95$ & Complex mixtures \\
\hline 22 & $3 g$ & $\mathrm{DCM}$ & $1: 3$ & $\mathrm{PhI}(\mathrm{OAc})_{2}$ & 48 & $<5$ & - \\
\hline 23 & $3 g$ & DCM & $1: 3$ & IBX & 48 & $<5$ & - \\
\hline 24 & $3 g$ & $\mathrm{DCM}$ & $1: 3$ & Dess-Martin & 48 & $<5$ & - \\
\hline 25 & $3 g$ & DCM & $1: 3$ & ${ }^{t} \mathrm{BuOOH}$ & 48 & $<5$ & - \\
\hline
\end{tabular}

Isolated yield was given in the parentheses. 


\section{General procedure for preparation of racemic samples}<smiles>O=C1[R1]=CC(=O)C=C1</smiles>

1a<smiles>[R]c1cc2ccccc2c([R])c1O</smiles>

2

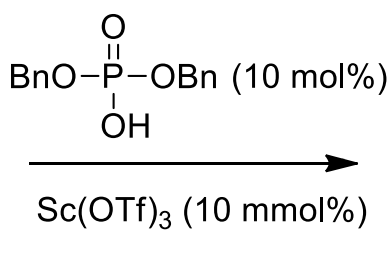

DCM, rt<smiles>[R]CC(=O)C1=CC(=O)C=CC1=O</smiles>

4

In an ordinary vial, quinone $1 \mathbf{a}(0.30 \mathrm{mmol})$ was added to a stirred mixture of dibenzyl phosphate (10 mol\%), $\mathrm{Sc}(\mathrm{OTf})_{3}(10 \mathrm{~mol} \%)$ and substituted 2-naphthol $2(0.10 \mathrm{mmol})$ in $\mathrm{CH}_{2} \mathrm{Cl}_{2}(1.0 \mathrm{~mL})$ at room temperature. The mixture was stirred at this temperature for the requisite amount of time as monitored by TLC. The solvent was removed under vacuum and residue was chromatographed on silica gel and fractions were collected and concentrated in vacuo to provide the pure desired products.

\section{General experimental procedure for dearomatization of 2-naphthols with quinone}<smiles>O=C1C=CC(=O)[Te]=[Te]1</smiles>

1a<smiles>[R]c1cc2ccccc2c([R])c1O</smiles>

2<smiles>[R]C1=CC(=O)C(C2([R])C(=O)C([R])=Cc3ccccc32)=CC1=O</smiles>

4

In an ordinary vial, quinone $1 \mathrm{a}(0.60 \mathrm{mmol})$ was added to a stirred mixture of 2-naphthols $2(0.20$ mmol) and catalyst $\mathbf{3 g}(0.02 \mathrm{mmol})$ in $\mathrm{CH}_{2} \mathrm{Cl}_{2}(2.0 \mathrm{~mL})$ at room temperature. The mixture was stirred at this temperature for the requisite amount of time as monitored by TLC. The solvent was removed under vacuum and residue was chromatographed on silica gel (petroleum ether/AcOEt 20:1 - 6:1) and fractions were collected and concentrated in vacuo to provide the pure desired products 4 . 


\section{Further Transformations of the Products}<smiles>CC1=Cc2ccccc2[C@@](C)(C2=CC(=O)C=CC2=O)C1=O</smiles>

4a, $98 \%$ ee
1. $\mathrm{NaBH}_{4}, \mathrm{THF} / \mathrm{CH}_{3} \mathrm{OH}, 20 \mathrm{~min}$

2.TFA, $\mathrm{CH}_{2} \mathrm{Cl}_{2}, 10 \mathrm{~min}$

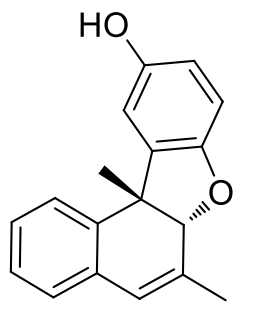

$\mathbf{5}, 75 \%$ yield $\mathrm{dr}=>20: 1,98 \%$ ee

In an ordinary vial, $\mathrm{NaBH}_{4}(0.90 \mathrm{mmol}, 4.5 \mathrm{eq})$ was added to a stirred mixture of (R)-2-(1,3-dimethyl-2-oxo-1,2-dihydronaphthalen-1-yl)cyclohexa-2,5-diene-1,4-dione $\quad$ 4a $\quad(0.20$ mmol) in THF $(0.5 \mathrm{~mL})$ at $0^{\circ} \mathrm{C}$, followed by the dropwise addition of $0.5 \mathrm{~mL}$ of $\mathrm{MeOH}$. The mixture was stirred at this temperature for $20 \mathrm{~min}$. The solvent was removed under vacuum and the residue was added TFA (trifluoroacetic acid, $0.2 \mathrm{~mL}$ ) at $0^{\circ} \mathrm{C}$. The mixture was stirred at this temperature for $10 \mathrm{~min}$. The solvent was removed under vacuum and residue was chromatographed on silica gel (petroleum ether/AcOEt 15:1) and fractions were collected and concentrated in vacuo to provide the pure desired products. 


\section{Characterization of 4 and 5}

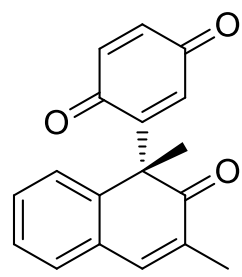

$4 a$

\section{(R)-2-(1,3-dimethyl-2-oxo-1,2-dihydronaphthalen-1-yl)cyclohexa-2,5-diene-1,4-dione, 4a}

Using $10 \mathrm{~mol} \%$ cat. $3 \mathrm{~g}$ at $\mathrm{rt}$ with $36 \mathrm{~h}, 43.9 \mathrm{mg}$ (78\% yield) of the pure product was obtained by silica gel column chromatography (petroleum/ethyl acetate $=20: 1-10: 1$ ) as a yellow solid; ${ }^{1} \mathbf{H}$ $\operatorname{NMR}\left(300 \mathrm{MHz}, \mathrm{CDCl}_{3}\right) \delta 7.41(\mathrm{~s}, 1 \mathrm{H}), 7.38-7.32(\mathrm{~m}, 1 \mathrm{H}), 7.31-7.18(\mathrm{~m}, 2 \mathrm{H}), 7.04(\mathrm{~d}, J=2.4$ $\mathrm{Hz}, 1 \mathrm{H}), 6.96-6.87(\mathrm{~m}, 1 \mathrm{H}), 6.77(\mathrm{dd}, J=10.1,2.4 \mathrm{~Hz}, 1 \mathrm{H}), 6.61(\mathrm{~d}, J=10.1 \mathrm{~Hz}, 1 \mathrm{H}), 2.06(\mathrm{~d}, J$ $=0.9 \mathrm{~Hz}, 3 \mathrm{H}), 1.50(\mathrm{~s}, 3 \mathrm{H}) .{ }^{13} \mathbf{C}$ NMR $\left(75 \mathrm{MHz}, \mathrm{CDCl}_{3}\right) \delta 200.7,187.5,185.7,151.8,143.6$, $141.3,136.7,136.1,133.8,131.4,129.3,129.1,129.0,127.3,125.2,53.1,26.5,16.0$; IR: 3314 , $2924,1663,1555,1373,1286,1097,1034,995,919,738 \mathrm{~cm}^{-1} ;[\alpha]_{\mathbf{D}}{ }^{\text {rt }}=-40^{\circ}\left(\mathrm{c}=1.00, \mathrm{CHCl}_{3}\right)$; HRMS (ESI): $\mathrm{C}_{18} \mathrm{H}_{14} \mathrm{O}_{3}+\mathrm{H}$, Calc: 279.1016, Found: 279.1017; HPLC: DAICEL CHIRALCEL IC, Hexane $/ \mathrm{EtOH}=1 / 1$, flow rate $=1.0 \mathrm{ml} / \mathrm{min}$, retention time: $\mathrm{t}_{\text {major }}=7.3, \mathrm{t}_{\text {minor }}=6.2,98 \%$ ee

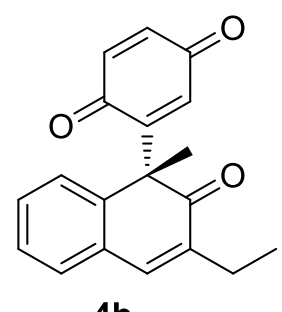

$4 b$

(R)-2-(3-ethyl-1-methyl-2-oxo-1,2-dihydronaphthalen-1-yl)cyclohexa-2,5-diene-1,4-dione, 4b

Using $10 \mathrm{~mol} \%$ cat. $3 \mathrm{~g}$ at $\mathrm{rt}$ with $10 \mathrm{~h}, 46 \mathrm{mg}$ (79 \% yield) of the pure product was obtained by silica gel column chromatography (petroleum/ethyl acetate $=20: 1-15: 1$ ) as a yellow solid; ${ }^{1} \mathbf{H}$ $\operatorname{NMR}\left(300 \mathrm{MHz}, \mathrm{CDCl}_{3}\right) \delta 7.43-7.30(\mathrm{~m}, 2 \mathrm{H}), 7.30-7.16(\mathrm{~m}, 2 \mathrm{H}), 7.03(\mathrm{~d}, J=2.4 \mathrm{~Hz}, 1 \mathrm{H})$, $6.98-6.84(\mathrm{~m}, 1 \mathrm{H}), 6.76(\mathrm{dd}, J=10.1,2.4 \mathrm{~Hz}, 1 \mathrm{H}), 6.61(\mathrm{~d}, J=10.1 \mathrm{~Hz}, 1 \mathrm{H}), 2.47(\mathrm{q}, J=7.5 \mathrm{~Hz}$, 2H), 1.49 (s, 3H), $1.18(\mathrm{t}, J=7.4 \mathrm{~Hz}, 3 \mathrm{H}) .{ }^{13} \mathbf{C}$ NMR $\left(75 \mathrm{MHz}, \mathrm{CDCl}_{3}\right) \delta 200.2,187.5,185.6$, $151.9,143.4,139.5,136.9,136.7,136.1,133.8,129.5,129.1,129.0,127.3,125.1,53.2,26.4,22.3$, 12.4; IR: 3312, 2927, 1657, 1457, 1385, 1337, 1286, 1096, 992, 917, $760 \mathrm{~cm}^{-1} ;[\boldsymbol{\alpha}]_{\mathbf{D}}{ }^{\text {rt }}=-42^{\circ}(\mathrm{c}=$ 
1.00, $\left.\mathrm{CHCl}_{3}\right) ; \mathrm{C}_{19} \mathrm{H}_{16} \mathrm{O}_{3}+\mathrm{H}$, Calc: 293.1172, Found: 293.1172; HPLC: DAICEL CHIRALCEL IC, Hexane $/ \mathrm{EtOH}=8 / 2$, flow rate $=1.0 \mathrm{ml} / \mathrm{min}$, retention time: $\mathrm{t}_{\text {major }}=11.0, \mathrm{t}_{\text {minor }}=8.2,98 \%$ ee

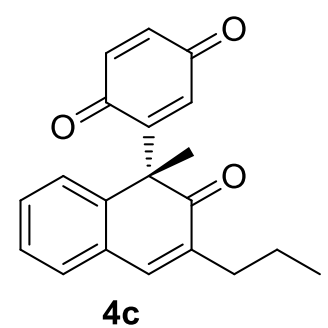

(R)-2-(1-methyl-2-oxo-3-propyl-1,2-dihydronaphthalen-1-yl)cyclohexa-2,5-diene-1,4-dione,

$4 \mathrm{c}$

Using $10 \mathrm{~mol} \%$ cat. $3 \mathrm{~g}$ at $\mathrm{rt}$ with $22 \mathrm{~h}, 43 \mathrm{mg}$ ( $71 \%$ yield) of the pure product was obtained by silica gel column chromatography (petroleum/ethyl acetate $=20: 1-15: 1$ ) as a yellow solid; ${ }^{1} \mathbf{H}$ NMR $\left(300 \mathrm{MHz}, \mathrm{CDCl}_{3}\right) \delta 7.42-7.32(\mathrm{~m}, 2 \mathrm{H}), 7.30-7.16(\mathrm{~m}, 2 \mathrm{H}), 7.03(\mathrm{~d}, J=2.4 \mathrm{~Hz}, 1 \mathrm{H})$, $6.97-6.85(\mathrm{~m}, 1 \mathrm{H}), 6.77(\mathrm{dd}, J=10.1,2.4 \mathrm{~Hz}, 1 \mathrm{H}), 6.61(\mathrm{~d}, J=10.1 \mathrm{~Hz}, 1 \mathrm{H}), 2.58-2.27(\mathrm{~m}$, 2H), $1.69-1.52(\mathrm{~m}, 2 \mathrm{H}), 1.49(\mathrm{~s}, 3 \mathrm{H}), 0.98(\mathrm{t}, J=7.4 \mathrm{~Hz}, 3 \mathrm{H}) .{ }^{13} \mathbf{C} \mathbf{N M R}\left(75 \mathrm{MHz}, \mathrm{CDCl}_{3}\right) \delta$ 200.3, 187.5, 185.6, 151.9, 143.5, 140.6, 136.7, 136.1, 135.4, 133.8, 129.5, 129.0, 129.0, 127.3, 125.1, 53.3, 31.4, 26.4, 21.6, 13.8; IR: 3313, 2926, 1659, 1456, 1382, 1286, 1096, 997, 919, 758 $\mathrm{cm}^{-1} ;[\boldsymbol{\alpha}]_{\mathbf{D}}{ }^{\mathbf{r t}}=-39^{\circ}\left(\mathrm{c}=1.00, \mathrm{CHCl}_{3}\right) ; \mathrm{C}_{20} \mathrm{H}_{19} \mathrm{O}_{3}+\mathrm{H}$, Calc: 307.1329, Found: 307.1331; HPLC: DAICEL CHIRALCEL IC, Hexane $/ \mathrm{EtOH}=8 / 2$, flow rate $=1.0 \mathrm{ml} / \mathrm{min}$, retention time: $\mathrm{t}_{\text {major }}=9.4$, $\mathrm{t}_{\mathrm{minor}}=7.4,98 \%$ ee.

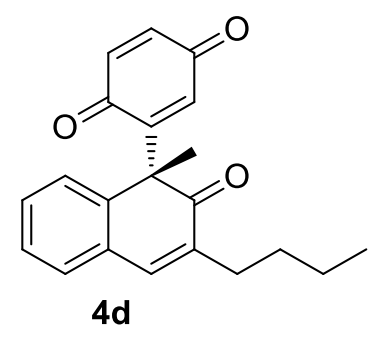

(R)-2-(3-butyl-1-methyl-2-oxo-1,2-dihydronaphthalen-1-yl)cyclohexa-2,5-diene-1,4-dione, 4d Using $10 \mathrm{~mol} \%$ cat. $3 \mathrm{~g}$ at $\mathrm{rt}$ with $48 \mathrm{~h}, 44 \mathrm{mg}$ (69\% yield) of the pure product was obtained by silica gel column chromatography (petroleum/ethyl acetate $=20: 1-15: 1$ ) as a yellow solid; ${ }^{1} \mathbf{H}$ NMR $\left(300 \mathrm{MHz}, \mathrm{CDCl}_{3}\right) \delta 7.41-7.30(\mathrm{~m}, 2 \mathrm{H}), 7.30-7.15(\mathrm{~m}, 2 \mathrm{H}), 7.03(\mathrm{~d}, J=2.4 \mathrm{~Hz}, 1 \mathrm{H})$, 
$6.96-6.85(\mathrm{~m}, 1 \mathrm{H}), 6.76(\mathrm{dd}, J=10.1,2.4 \mathrm{~Hz}, 1 \mathrm{H}), 6.61(\mathrm{~d}, J=10.1 \mathrm{~Hz}, 1 \mathrm{H}), 2.58-2.32(\mathrm{~m}$, 2H), $1.62-1.46(\mathrm{~m}, 5 \mathrm{H}), 1.45-1.37(\mathrm{~m}, 2 \mathrm{H}), 0.94(\mathrm{t}, J=7.2 \mathrm{~Hz}, 3 \mathrm{H}) .{ }^{13} \mathbf{C}$ NMR $(75 \mathrm{MHz}$, $\left.\mathrm{CDCl}_{3}\right) \delta 200.3,187.5,185.6,151.9,143.5,140.4,136.7,136.0,135.7,133.8,129.4,129.1,129.0$, 127.3, 125.1, 53.3, 30.5, 29.0, 26.4, 22.4, 13.9; IR: 3312, 2928, 1659, 1456, 1382, 1285, 1202 , 1097, 995, 919, $759 \mathrm{~cm}^{-1} ;[\boldsymbol{\alpha}]_{\mathbf{D}}{ }^{\mathrm{rt}}=+35^{\circ}\left(\mathrm{c}=1.00, \mathrm{CHCl}_{3}\right) ; \mathrm{C}_{21} \mathrm{H}_{20} \mathrm{O}_{3}+\mathrm{H}$, Calc: 321.1485 , Found: 321.1486; HPLC: DAICEL CHIRALCEL IC, Hexane $/ \mathrm{EtOH}=6 / 4$, flow rate $=1.0 \mathrm{ml} / \mathrm{min}$, retention time: $\mathrm{t}_{\text {major }}=6.3, \mathrm{t}_{\text {minor }}=5.2,96 \%$ ee.

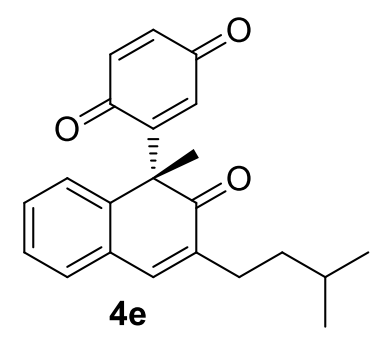

(R)-2-(3-isopentyl-1-methyl-2-oxo-1,2-dihydronaphthalen-1-yl)cyclohexa-2,5-diene-1,4-dione, $4 e$

Using $10 \mathrm{~mol} \%$ cat. $3 \mathrm{~g}$ at $\mathrm{rt}$ with $37 \mathrm{~h}, 45 \mathrm{mg}$ ( $68 \%$ yield) of the pure product was obtained by silica gel column chromatography (petroleum/ethyl acetate $=20: 1-15: 1$ ) as a yellow solid; ${ }^{1} \mathbf{H}$ NMR $\left(300 \mathrm{MHz}, \mathrm{CDCl}_{3}\right) \delta 7.45-7.31(\mathrm{~m}, 2 \mathrm{H}), 7.31-7.14(\mathrm{~m}, 2 \mathrm{H}), 7.03(\mathrm{~d}, J=2.4 \mathrm{~Hz}, 1 \mathrm{H})$, $6.97-6.85(\mathrm{~m}, 1 \mathrm{H}), 6.76(\mathrm{dd}, J=10.1,2.4 \mathrm{~Hz}, 1 \mathrm{H}), 6.61(\mathrm{~d}, J=10.1 \mathrm{~Hz}, 1 \mathrm{H}), 2.64-2.25(\mathrm{~m}$, 2H), $1.76-1.54(\mathrm{~m}, 1 \mathrm{H}), 1.54-1.34(\mathrm{~m}, 5 \mathrm{H}), 0.95(\mathrm{~d}, J=6.6 \mathrm{~Hz}, 6 \mathrm{H}) .{ }^{13} \mathbf{C}$ NMR $(75 \mathrm{MHz}$, $\left.\mathrm{CDCl}_{3}\right) \delta 200.3,187.5,185.6,151.9,143.5,140.3,136.7,136.1,136.0,133.8,129.4,129.1,129.0$ 127.3, 125.1, 53.3, 37.4, 27.9, 27.3, 26.4, 22.5, 22.5; IR: 3314, 2925, 1659, 1457, 1383, 1285, 1097, 997, 918, $758 \mathrm{~cm}^{-1} ;[\boldsymbol{\alpha}]_{\mathbf{D}}{ }^{\mathbf{r t}}=-35^{\circ}\left(\mathrm{c}=1.00, \mathrm{CHCl}_{3}\right) ; \mathrm{C}_{22} \mathrm{H}_{22} \mathrm{O}_{3}+\mathrm{H}$, Calc: 335.1642 , Found: 335.1644; HPLC: DAICEL CHIRALCEL IC, Hexane $/ \mathrm{PrOH}=8 / 2$, flow rate $=1.0 \mathrm{ml} / \mathrm{min}$, retention time: $\mathrm{t}_{\text {major }}=7.8, \mathrm{t}_{\text {minor }}=6.0,96 \%$ ee.

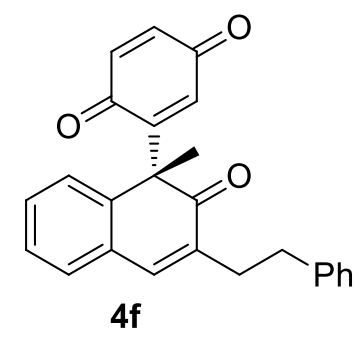


(R)-2-(1-methyl-2-oxo-3-phenethyl-1,2-dihydronaphthalen-1-yl)cyclohexa-2,5-diene-1,4-dion

e, $4 \mathbf{f}$

Using $10 \mathrm{~mol} \%$ cat. $3 \mathrm{~g}$ at rt with $21 \mathrm{~h}, 52 \mathrm{mg}$ (70\% yield) of the pure product was obtained by silica gel column chromatography (petroleum/ethyl acetate $=20: 1-15: 1$ ) as a yellow solid; ${ }^{1} \mathbf{H}$ NMR $\left(300 \mathrm{MHz}, \mathrm{CDCl}_{3}\right) \delta 7.35-7.09(\mathrm{~m}, 9 \mathrm{H}), 7.03(\mathrm{~d}, J=2.4 \mathrm{~Hz}, 1 \mathrm{H}), 6.96-6.85(\mathrm{~m}, 1 \mathrm{H})$, $6.76(\mathrm{dd}, J=10.0,2.4 \mathrm{~Hz}, 1 \mathrm{H}), 6.62(\mathrm{~d}, J=10.1 \mathrm{~Hz}, 1 \mathrm{H}), 2.96-2.80(\mathrm{~m}, 2 \mathrm{H}), 2.80-2.65(\mathrm{~m}$, 2H), $1.46(\mathrm{~s}, 3 \mathrm{H}) .{ }^{13} \mathrm{C}$ NMR $\left(75 \mathrm{MHz}, \mathrm{CDCl}_{3}\right) \delta 200.2,187.4,185.6,151.8,143.6,141.4,141.3$, 136.7, 136.1, 134.3, 133.8, 129.5, 129.2, 128.8, 128.6, 128.3, 127.3, 125.9, 125.1, 53.3, 34.6, 31.6, 26.4; IR: $3312,2925,1661,1454,1383,1286,1202,1096,995,919,757 \mathrm{~cm}^{-1} ;[\boldsymbol{\alpha}]_{\mathbf{D}}{ }^{\mathbf{r t}}=-39^{\circ}(\mathrm{c}=$ 1.00, $\mathrm{CHCl}_{3}$ ); $\mathrm{C}_{25} \mathrm{H}_{20} \mathrm{O}_{3}+\mathrm{H}$, Calc: 369.1485, Found: 369.1491; HPLC: DAICEL CHIRALCEL IA, Hexane $/ \mathrm{PrOH}=8 / 2$, flow rate $=1.0 \mathrm{ml} / \mathrm{min}$, retention time: $\mathrm{t}_{\text {major }}=18.3, \mathrm{t}_{\text {minor }}=8.7,99 \%$ ee

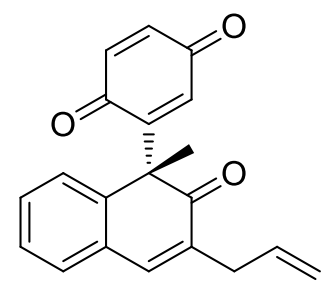

$4 \mathrm{~g}$

(R)-2-(3-allyl-1-methyl-2-oxo-1,2-dihydronaphthalen-1-yl)cyclohexa-2,5-diene-1,4-dione, $4 \mathrm{~g}$

Using $10 \mathrm{~mol} \%$ cat. $3 \mathrm{~g}$ at $\mathrm{rt}$ with $24 \mathrm{~h}, 38 \mathrm{mg}$ (63\% yield) of the pure product was obtained by silica gel column chromatography (petroleum/ethyl acetate $=20: 1-15: 1$ ) as a yellow solid; ${ }^{1} \mathbf{H}$ NMR (300 MHz, $\left.\mathrm{CDCl}_{3}\right) \delta 7.45-7.33(\mathrm{~m}, 2 \mathrm{H}), 7.33-7.18(\mathrm{~m}, 2 \mathrm{H}), 7.04(\mathrm{~d}, J=2.4 \mathrm{~Hz}, 1 \mathrm{H})$, $6.96-6.86(\mathrm{~m}, 1 \mathrm{H}), 6.77(\mathrm{dd}, J=10.1,2.4 \mathrm{~Hz}, 1 \mathrm{H}), 6.62(\mathrm{~d}, J=10.1 \mathrm{~Hz}, 1 \mathrm{H}), 6.04-5.86(\mathrm{~m}$, 1H), $5.54-4.68(\mathrm{~m}, 2 \mathrm{H}), 3.55-2.77(\mathrm{~m}, 2 \mathrm{H}), 1.50(\mathrm{~s}, 3 \mathrm{H}) .{ }^{13} \mathbf{C} \mathbf{N M R}\left(75 \mathrm{MHz}, \mathrm{CDCl}_{3}\right) \delta 199.8$, $187.5,185.6,151.8,143.6,141.2,136.7,136.1,134.9,133.8,133.5,129.7,129.3,128.9,127.4$, 125.2, 117.2, 53.3, 33.2, 26.5; IR: 3383, 2924, 1744, 1657, 1460, 1380, 1264, 1097, 918, $742 \mathrm{~cm}^{-1}$; $[\boldsymbol{\alpha}]_{\mathbf{D}}^{\mathbf{r t}}=-39^{\circ}\left(\mathrm{c}=1.00, \mathrm{CHCl}_{3}\right) ;$ HRMS (ESI): $\mathrm{C}_{20} \mathrm{H}_{16} \mathrm{O}_{3}+\mathrm{H}$, Calc: 305.1172, Found: 305.1169; HPLC: DAICEL CHIRALCEL IC, Hexane/iPrOH $=9 / 1$, flow rate $=1.0 \mathrm{ml} / \mathrm{min}$, retention time: $\mathrm{t}_{\text {major }}=16.5, \mathrm{t}_{\text {minor }}=14.7,93 \%$ ee. 


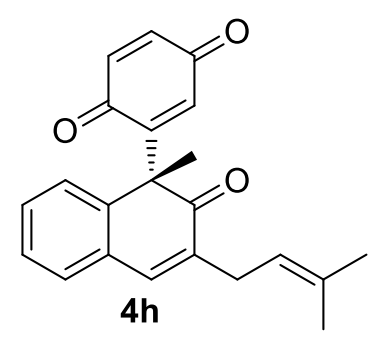

(R)-2-(1-methyl-3-(3-methylbut-2-en-1-yl)-2-oxo-1,2-dihydronaphthalen-1-yl)cyclohexa-2,5-d iene-1,4-dione, $4 \mathrm{~h}$

Using $10 \mathrm{~mol} \%$ cat. $3 \mathrm{~g}$ at $\mathrm{rt}$ with $37 \mathrm{~h}, 41 \mathrm{mg}$ (61\% yield) of the pure product was obtained by silica gel column chromatography (petroleum/ethyl acetate $=30: 1-20: 1$ ) as a yellow solid; ${ }^{1} \mathbf{H}$ NMR $\left(300 \mathrm{MHz}, \mathrm{CDCl}_{3}\right) \delta 7.43-7.34(\mathrm{~m}, 1 \mathrm{H}), 7.31(\mathrm{~s}, 1 \mathrm{H}), 7.29-7.16(\mathrm{~m}, 2 \mathrm{H}), 7.03(\mathrm{~d}, J=2.4$ Hz, 1H), $6.98-6.87(\mathrm{~m}, 1 \mathrm{H}), 6.77(\mathrm{dd}, J=10.1,2.4 \mathrm{~Hz}, 1 \mathrm{H}), 6.61(\mathrm{~d}, J=10.1 \mathrm{~Hz}, 1 \mathrm{H}), 5.36-$ $5.21(\mathrm{~m}, 1 \mathrm{H}), 3.47-2.77(\mathrm{~m}, 2 \mathrm{H}), 1.80(\mathrm{~s}, 3 \mathrm{H}), 1.70(\mathrm{~s}, 3 \mathrm{H}), 1.50(\mathrm{~s}, 3 \mathrm{H}) .{ }^{13} \mathbf{C} \mathbf{N M R}(75 \mathrm{MHz}$, $\left.\mathrm{CDCl}_{3}\right) \delta 200.3,187.5,185.6,151.9,143.5,140.3,136.7,136.1,134.6,134.5,133.8,129.6,129.1$, 129.1, 127.3, 125.1, 120.1, 53.4, 27.5, 26.4, 25.8, 17.8; IR: 3405, 2924, 1657, 1448, 1382, 1286, 1097, 998, 919, $759 \mathrm{~cm}^{-1} ;[\boldsymbol{\alpha}]_{\mathbf{D}}{ }^{\mathbf{r t}}=+25^{\circ}\left(\mathrm{c}=1.00, \mathrm{CHCl}_{3}\right) ; \mathrm{C}_{22} \mathrm{H}_{20} \mathrm{O}_{3}+\mathrm{H}$, Calc: 333.1485 , Found: 333.1484; HPLC: DAICEL CHIRALCEL AD, Hexane $/$ EtOH $=7 / 3$, flow rate $=1.0 \mathrm{ml} / \mathrm{min}$, retention time: $\mathrm{t}_{\text {major }}=8.3, \mathrm{t}_{\text {minor }}=6.8,96 \%$ ee.

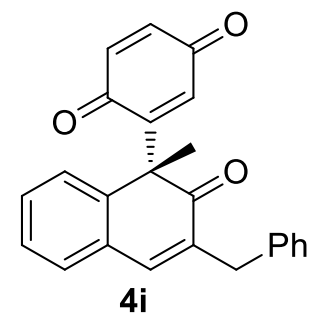

®-2-(3-benzyl-1-methyl-2-oxo-1,2-dihydronaphthalen-1-yl)cyclohexa-2,5-diene-1,4-dione, 4i Using $10 \mathrm{~mol} \%$ cat. $3 \mathrm{~g}$ at $\mathrm{rt}$ with $24 \mathrm{~h}, 51 \mathrm{mg}$ (72\% yield) of the pure product was obtained by silica gel column chromatography (petroleum/ethyl acetate $=20: 1-10: 1$ ) as a yellow solid; ${ }^{1} \mathbf{H}$ NMR $\left(300 \mathrm{MHz}, \mathrm{CDCl}_{3}\right) \delta 7.37-7.15(\mathrm{~m}, 9 \mathrm{H}), 7.02(\mathrm{~d}, J=2.4 \mathrm{~Hz}, 1 \mathrm{H}), 6.97-6.88(\mathrm{~m}, 1 \mathrm{H})$, $6.75(\mathrm{dd}, J=10.1,2.4 \mathrm{~Hz}, 1 \mathrm{H}), 6.61(\mathrm{~d}, J=10.1 \mathrm{~Hz}, 1 \mathrm{H}), 3.77(\mathrm{dd}, J=37.9,15.8 \mathrm{~Hz}, 2 \mathrm{H}), 1.46$ (s, 3H). ${ }^{13} \mathrm{C}$ NMR $\left(75 \mathrm{MHz}, \mathrm{CDCl}_{3}\right) \delta 199.9,187.4,185.6,151.7,143.6,141.7,138.9,136.7,136.1$, 134.8, 133.8, 129.8, 129.3, 129.1, 128.8, 128.5, 127.3, 126.3, 125.1, 53.4, 35.2, 26.3; IR: 3314, $2926,1663,1454,1382,1286,1096,998,919,739 \mathrm{~cm}^{-1} ;[\boldsymbol{\alpha}]_{\mathbf{D}}{ }^{\mathbf{r t}}=-10^{\circ}\left(\mathrm{c}=1.00, \mathrm{CHCl}_{3}\right) ; \mathrm{HRMS}$ 
(ESI): $\mathrm{C}_{24} \mathrm{H}_{18} \mathrm{O}_{3}+\mathrm{H}$, Calc: 355.1329, Found: 355.1331; HPLC: DAICEL CHIRALCEL IC, Hexane $/ \mathrm{EtOH}=1 / 1$, flow rate $=1.0 \mathrm{ml} / \mathrm{min}$, retention time: $\mathrm{t}_{\text {major }}=6.4, \mathrm{t}_{\text {minor }}=8.5,95 \%$ ee

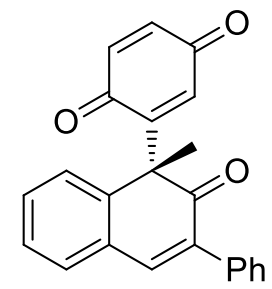

4j

(R)-2-(1-methyl-2-oxo-3-phenyl-1,2-dihydronaphthalen-1-yl)cyclohexa-2,5-diene-1,4-dione,

\section{$4 \mathbf{j}$}

Using $10 \mathrm{~mol} \%$ cat. $3 \mathrm{~g}$ at $\mathrm{rt}$ with $48 \mathrm{~h}, 15 \mathrm{mg}$ ( $22 \%$ yield) of the pure product was obtained by silica gel column chromatography (petroleum/ethyl acetate $=20: 1-15: 1$ ) as a yellow solid; ${ }^{1} \mathbf{H}$ $\operatorname{NMR}\left(300 \mathrm{MHz}, \mathrm{CDCl}_{3}\right) \delta 7.68(\mathrm{~s}, 1 \mathrm{H}), 7.55(\mathrm{dd}, J=8.0,1.5 \mathrm{~Hz}, 2 \mathrm{H}), 7.52-7.45(\mathrm{~m}, 1 \mathrm{H}), 7.45$ $-7.34(\mathrm{~m}, 3 \mathrm{H}), 7.34-7.28(\mathrm{~m}, 2 \mathrm{H}), 7.07(\mathrm{~d}, J=2.4 \mathrm{~Hz}, 1 \mathrm{H}), 6.99-6.91(\mathrm{~m}, 1 \mathrm{H}), 6.79(\mathrm{dd}, J=$ 10.1, $2.4 \mathrm{~Hz}, 1 \mathrm{H}), 6.64(\mathrm{~d}, J=10.1 \mathrm{~Hz}, 1 \mathrm{H}), 1.62(\mathrm{~s}, 3 \mathrm{H}) .{ }^{13} \mathbf{C} \mathbf{N M R}\left(75 \mathrm{MHz}, \mathrm{CDCl}_{3}\right) \delta 199.0$, $187.5,185.7,151.9,143.9,142.3,136.7,136.2,135.4,134.3,133.8,130.5,129.9,129.0,128.8$, 128.2, 128.2, 127.6, 125.3, 54.1, 26.5; IR: 3398, 2924, 1658, 1456, 1375, 1285, 1096, 1028, 919 , $740 \mathrm{~cm}^{-1} ;[\boldsymbol{\alpha}]_{\mathbf{D}}{ }^{\mathbf{r t}}=-24^{\circ}\left(\mathrm{c}=1.00, \mathrm{CHCl}_{3}\right) ; \mathrm{C}_{23} \mathrm{H}_{16} \mathrm{O}_{3}+\mathrm{H}$, Calc: 341.1172 , Found: 341.1174; HPLC: DAICEL CHIRALCEL IC, Hexane $/ \mathrm{EtOH}=9 / 1$, flow rate $=1.0 \mathrm{ml} / \mathrm{min}$, retention time: $\mathrm{t}_{\text {major }}$ $=20.3, \mathrm{t}_{\text {minor }}=18.1,91 \%$ ee.<smiles>CC12C(=O)C=CC(=O)C1(C)c1ccccc1C=CC2=O</smiles>

$4 \mathbf{k}$

\section{(R)-2-(1-methyl-2-oxo-1,2-dihydronaphthalen-1-yl)cyclohexa-2,5-diene-1,4-dione, 4k}

Using $10 \mathrm{~mol} \%$ cat. $3 \mathrm{~g}$ at rt with $60 \mathrm{~h}, 15 \mathrm{mg}$ ( $28 \%$ yield) of the pure product was obtained by silica gel column chromatography (petroleum/ethyl acetate $=20: 1-15: 1$ ) as a yellow solid; ${ }^{1} \mathbf{H}$ NMR $\left(300 \mathrm{MHz}, \mathrm{CDCl}_{3}\right) \delta 7.59(\mathrm{~d}, J=9.9 \mathrm{~Hz}, 1 \mathrm{H}), 7.51-7.40(\mathrm{~m},, 1 \mathrm{H}), 7.35-7.28(\mathrm{~m}, 2 \mathrm{H})$, 
$7.05(\mathrm{~d}, J=2.4 \mathrm{~Hz}, 1 \mathrm{H}), 6.99-6.90(\mathrm{~m}, 1 \mathrm{H}), 6.78(\mathrm{dd}, J=10.1,2.4 \mathrm{~Hz}, 1 \mathrm{H}), 6.63(\mathrm{~d}, J=10.1 \mathrm{~Hz}$ 1H), $6.29(\mathrm{~d}, J=9.9 \mathrm{~Hz}, 1 \mathrm{H}), 1.53(\mathrm{~s}, 3 \mathrm{H}) .{ }^{13} \mathbf{C} \mathbf{N M R}\left(75 \mathrm{MHz}, \mathrm{CDCl}_{3}\right) \delta$ 200.5, 187.4., 185.6, 151.5, 145.0, 144.2, 136.7, 136.2, 134.0, 130.3, 130.3, 128.4, 127.5, 125.5, 124.0, 53.4, 26.4. IR: $3313,2926,1656,1598,1452,1398,1338,1287,1097,919,830,761,607 \mathrm{~cm}^{-1} ;[\boldsymbol{\alpha}]_{\mathbf{D}}{ }^{\mathrm{rt}}=-2^{\circ}(\mathrm{c}=$ 1.00, $\mathrm{CHCl}_{3}$ ); HRMS (ESI): $\mathrm{C}_{17} \mathrm{H}_{12} \mathrm{O}_{3}+\mathrm{H}$, Calc: 265.0859, Found: 265.0857; HPLC: DAICEL CHIRALCEL IA, Hexane $/ \mathrm{PrOH}=8 / 2$, flow rate $=1.0 \mathrm{ml} / \mathrm{min}$, retention time: $\mathrm{t}_{\text {major }}=14.9, \mathrm{t}_{\text {minor }}=$ $13.3,58 \%$ ee.

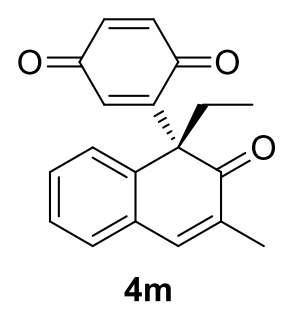

(R)-2-(1-ethyl-3-methyl-2-oxo-1,2-dihydronaphthalen-1-yl)cyclohexa-2,5-diene-1,4-dione, 4m Using $10 \mathrm{~mol} \%$ cat. $3 \mathrm{~g}$ at $\mathrm{rt}$ with $24 \mathrm{~h}, 41 \mathrm{mg}$ (71\% yield) of the pure product was obtained by silica gel column chromatography (petroleum/ethyl acetate $=20: 1-15: 1$ ) as a yellow solid; ${ }^{1} \mathbf{H}$ $\operatorname{NMR}\left(300 \mathrm{MHz}, \mathrm{CDCl}_{3}\right) \delta 7.38(\mathrm{~s}, 1 \mathrm{H}), 7.36-7.31(\mathrm{~m}, 1 \mathrm{H}), 7.30-7.17(\mathrm{~m}, 2 \mathrm{H}), 7.04(\mathrm{~d}, J=2.4$ Hz, 1H), $7.01-6.83(\mathrm{~m}, 1 \mathrm{H}), 6.73(\mathrm{dd}, J=10.1,2.4 \mathrm{~Hz}, 1 \mathrm{H}), 6.57(\mathrm{~d}, J=10.1 \mathrm{~Hz}, 1 \mathrm{H}), 2.27-$ $2.08(\mathrm{~m}, 1 \mathrm{H}), 2.04(\mathrm{~d}, J=1.1 \mathrm{~Hz}, 3 \mathrm{H}), 2.00-1.83(\mathrm{~m}, 1 \mathrm{H}), 0.59(\mathrm{t}, J=7.4 \mathrm{~Hz}, 3 \mathrm{H}) .{ }^{13} \mathbf{C} \mathbf{~ N M R}$ $\left(75 \mathrm{MHz}, \mathrm{CDCl}_{3}\right) \delta 200.9,187.6,185.7,152.6,141.8,141.5,136.7,135.8,133.7,133.4,131.1$, 128.8, 128.8, 127.3, 125.6, 56.1, 34.7, 15.6, 7.3; IR: 3319, 2925, 1663, 1460, 1378, 1265, 1094 , $909,740 \mathrm{~cm}^{-1} ;[\boldsymbol{\alpha}]_{\mathbf{D}}{ }^{\text {rt }}=-73^{\circ}\left(\mathrm{c}=1.00, \mathrm{CHCl}_{3}\right)$; HRMS (ESI): $\mathrm{C}_{19} \mathrm{H}_{16} \mathrm{O}_{3}+\mathrm{H}$, Calc: 293.1172 , Found: 293.1171; HPLC: DAICEL CHIRALCEL IC, Hexane $/ \mathrm{EtOH}=9 / 1$, flow rate $=1.0 \mathrm{ml} / \mathrm{min}$, retention time: $\mathrm{t}_{\text {major }}=20.2, \mathrm{t}_{\text {minor }}=17.2,98 \%$ ee.

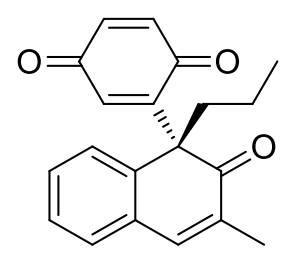

$4 n$

(R)-2-(3-methyl-2-oxo-1-propyl-1,2-dihydronaphthalen-1-yl)cyclohexa-2,5-diene-1,4-dione, $4 n$ 
Using $10 \mathrm{~mol} \%$ cat. $3 \mathrm{~g}$ at $\mathrm{rt}$ with $10 \mathrm{~h}, 41 \mathrm{mg}$ ( $67 \%$ yield) of the pure product was obtained by silica gel column chromatography (petroleum/ethyl acetate $=20: 1-15: 1$ ) as a yellow solid; ${ }^{1} \mathbf{H}$ NMR $\left(300 \mathrm{MHz}, \mathrm{CDCl}_{3}\right) \delta 7.37(\mathrm{~s}, 1 \mathrm{H}), 7.35-7.28(\mathrm{~m}, 1 \mathrm{H}), 7.28-7.18(\mathrm{~m}, 2 \mathrm{H}), 7.05(\mathrm{~d}, J=2.4$ Hz, 1H), $6.97-6.88(\mathrm{~m}, 1 \mathrm{H}), 6.73(\mathrm{dd}, J=10.1,2.4 \mathrm{~Hz}, 1 \mathrm{H}), 6.57(\mathrm{~d}, J=10.1 \mathrm{~Hz}, 1 \mathrm{H}), 2.15-$ $1.95(\mathrm{~m}, 4 \mathrm{H}), 1.80(\mathrm{td}, J=12.0,3.2 \mathrm{~Hz}, 1 \mathrm{H}), 1.19-0.98(\mathrm{~m}, 1 \mathrm{H}), 0.91-0.79(\mathrm{~m}, 1 \mathrm{H}), 0.75(\mathrm{t}, J=$

$6.3 \mathrm{~Hz}, 3 \mathrm{H}) .{ }^{13} \mathbf{C} \mathbf{N M R}\left(75 \mathrm{MHz}, \mathrm{CDCl}_{3}\right) \delta 200.9,187.7,185.7,152.6,142.2,141.4,136.7,135.8$, 133.6, 133.4, 130.9, 128.9, 128.8, 127.3, 125.6, 55.9, 43.9, 15.9, 15.7, 14.3; IR: 3312, 2925, 1657, $1458,1378,1281,1090,902,761 \mathrm{~cm}^{-1} ;[\alpha]_{\mathbf{D}}{ }^{\text {rt }}=-56^{\circ}\left(\mathrm{c}=1.00, \mathrm{CHCl}_{3}\right) ; \mathrm{C}_{20} \mathrm{H}_{18} \mathrm{O}_{3}+\mathrm{H}$, Calc: 307.1329, Found: 307.1328; HPLC: DAICEL CHIRALCEL IC, Hexane/EtOH $=8 / 2$, flow rate $=$ $1.0 \mathrm{ml} / \mathrm{min}$, retention time: $\mathrm{t}_{\text {major }}=8.9, \mathrm{t}_{\text {minor }}=7.0,99 \%$ ee.

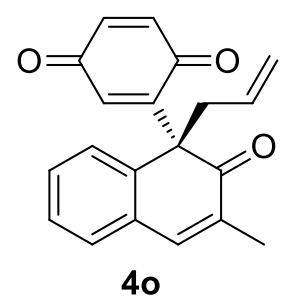

(R)-2-(1-allyl-3-methyl-2-oxo-1,2-dihydronaphthalen-1-yl)cyclohexa-2,5-diene-1,4-dione, 40 Using $10 \mathrm{~mol} \%$ cat. $3 \mathrm{~g}$ at $\mathrm{rt}$ with $24 \mathrm{~h}, 38 \mathrm{mg}$ (63\% yield) of the pure product was obtained by silica gel column chromatography (petroleum/ethyl acetate $=20: 1-15: 1$ ) as a yellow solid; ${ }^{1} \mathbf{H}$ NMR $\left(300 \mathrm{MHz}, \mathrm{CDCl}_{3}\right) \delta 7.38-7.29(\mathrm{~m}, 2 \mathrm{H}), 7.28-7.20(\mathrm{~m}, 2 \mathrm{H}), 7.04(\mathrm{~d}, J=2.4 \mathrm{~Hz}, 1 \mathrm{H})$, $6.97(\mathrm{dd}, J=6.1,2.7 \mathrm{~Hz}, 1 \mathrm{H}), 6.75(\mathrm{dd}, J=10.1,2.4 \mathrm{~Hz}, 1 \mathrm{H}), 6.59(\mathrm{~d}, J=10.1 \mathrm{~Hz}, 1 \mathrm{H}), 5.38-$ $5.18(\mathrm{~m}, 1 \mathrm{H}), 4.94-4.75(\mathrm{~m}, 2 \mathrm{H}), 2.71(\mathrm{ddd}, J=18.9,12.4,7.3 \mathrm{~Hz}, 2 \mathrm{H}), 2.00(\mathrm{~d}, J=1.3 \mathrm{~Hz}, 3 \mathrm{H})$. ${ }^{13}$ C NMR $\left(75 \mathrm{MHz}, \mathrm{CDCl}_{3}\right) \delta 200.2,187.5,185.6,151.9,141.5,141.4,136.7,135.9,133.6,130.7$, 130.0, 128.9, 128.9, 127.5, 125.7, 119.3, 56.0, 45.5, 15.6; IR: 3317, 2924, 1660, 1460, 1379, 1265 , 1092, 1017, 913, $741 \mathrm{~cm}^{-1} ;[\boldsymbol{\alpha}]_{\mathbf{D}}{ }^{\mathrm{rt}}=-75^{\circ}\left(\mathrm{c}=1.00, \mathrm{CHCl}_{3}\right)$; HRMS (ESI): $\mathrm{C}_{20} \mathrm{H}_{16} \mathrm{O}_{3}+\mathrm{H}$, Calc: 305.1172, Found: 305.1172; HPLC: DAICEL CHIRALCEL IC, Hexane/iPrOH = 9/1, flow rate = $1.0 \mathrm{ml} / \mathrm{min}$, retention time: $\mathrm{t}_{\text {major }}=16.8, \mathrm{t}_{\text {minor }}=14.2,95 \%$ ee. 


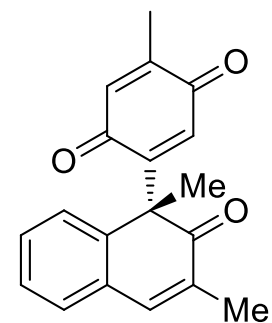

$4 p$

(R)-2-(1,3-dimethyl-2-oxo-1,2-dihydronaphthalen-1-yl)-5-methylcyclohexa-2,5-diene-1,4-dio ne, 4 p.

Using $10 \mathrm{~mol} \%$ cat. $3 \mathrm{~g}$ at $\mathrm{rt}$ with $48 \mathrm{~h}, 20 \mathrm{mg}$ (35\% yield) of the pure product was obtained by silica gel column chromatography (petroleum/ethyl acetate $=20: 1-15: 1$ ) as a yellow solid; ${ }^{1} \mathbf{H}$ NMR (300 MHz, $\left.\mathrm{CDCl}_{3}\right) \delta 7.38(\mathrm{~s}, 1 \mathrm{H}), 7.36-7.30(\mathrm{~m}, 1 \mathrm{H}), 7.29-7.15(\mathrm{~m}, 2 \mathrm{H}), 7.01(\mathrm{~s}, 1 \mathrm{H})$, $6.95-6.88(\mathrm{~m}, 1 \mathrm{H}), 6.44(\mathrm{~d}, J=1.6 \mathrm{~Hz}, 1 \mathrm{H}), 2.05(\mathrm{t}, J=1.6 \mathrm{~Hz}, 6 \mathrm{H}), 1.48(\mathrm{~s}, 3 \mathrm{H}) .{ }^{13} \mathbf{C ~ N M R}(75$ $\left.\mathrm{MHz}, \mathrm{CDCl}_{3}\right) \delta 200.7,188.0,185.9,151.9,145.6,143.8,141.2,133.8,133.5,131.5,129.3,129.1$, 129.0, 127.3, 125.3, 52.9, 26.6, 16.0, 15.4. IR: 3280, 2924, 1743, 1656, 1440, 1376, 1342, 1249 , $1203,1102,1033,918,760,737 \mathrm{~cm}^{-1} ;[\alpha]_{\mathbf{D}}{ }^{\mathbf{r t}}=-4^{\circ}\left(\mathrm{c}=1.00, \mathrm{CHCl}_{3}\right) ; \mathrm{HRMS}$ ESI): $\mathrm{C}_{19} \mathrm{H}_{16} \mathrm{O}_{3}+\mathrm{H}$, Calc: 293.1172, Found: 293.1184; HPLC: DAICEL CHIRALCEL IC, Hexane/iPrOH = 8/2, flow rate $=1.0 \mathrm{ml} / \mathrm{min}$, retention time: $\mathrm{t}_{\text {major }}=11.3, \mathrm{t}_{\mathrm{minor}}=8.7,82 \%$ ee.

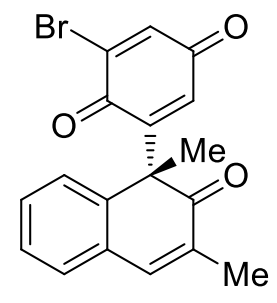

$4 q$

(R)-2-bromo-6-(1,3-dimethyl-2-oxo-1,2-dihydronaphthalen-1-yl)cyclohexa-2,5-diene-1,4-dion e, $4 \mathbf{q}$

Using $10 \mathrm{~mol} \%$ cat. $3 \mathrm{~g}$ at $\mathrm{rt}$ with $36 \mathrm{~h}, 15 \mathrm{mg}$ (21\% yield) of the pure product was obtained by silica gel column chromatography (petroleum/ethyl acetate $=15: 1-10: 1$ ) as a yellow solid; ${ }^{1} \mathbf{H}$ NMR $\left(300 \mathrm{MHz}, \mathrm{CDCl}_{3}\right) \delta 7.40(\mathrm{~s}, 1 \mathrm{H}), 7.36(\mathrm{dd}, J=7.3,1.7 \mathrm{~Hz}, 1 \mathrm{H}), 7.31-7.22(\mathrm{~m}, 2 \mathrm{H}), 7.21$ $(\mathrm{s}, 1 \mathrm{H}), 7.12(\mathrm{~s}, 1 \mathrm{H}), 6.93-6.85(\mathrm{~m}, 1 \mathrm{H}), 2.05(\mathrm{~d}, J=1.3 \mathrm{~Hz}, 3 \mathrm{H}), 1.50(\mathrm{~s}, 3 \mathrm{H}) .{ }^{\mathbf{1 3}} \mathbf{C} \mathbf{~ N M R}(75$ $\left.\mathrm{MHz}, \mathrm{CDCl}_{3}\right) \delta 200.4,183.1,179.5,152.4,143.3,141.5,138.1,137.3,133.2,131.4,129.4,129.1$, 129.0, 127.5, 125.2, 53.2, 26.6, 15.9. IR: 3313, 2925, 1656, 1745, 1665, 1655, 1594, 1459, 1377 , 
1197, 1035, 1001, 912, 759, $737 \mathrm{~cm}^{-1} ;[\boldsymbol{\alpha}]_{\mathbf{D}}{ }^{\mathrm{rt}}=-15^{\circ}\left(\mathrm{c}=1.00, \mathrm{CHCl}_{3}\right) ;$ HRMS (ESI): $\mathrm{C}_{18} \mathrm{H}_{13} \mathrm{BrO}_{3}+\mathrm{Na}$, Calc: 378.9940, Found: 378.9951; HPLC: DAICEL CHIRALCEL IA, Hexane $/ \mathrm{iPrOH}=8 / 2$, flow rate $=1.0 \mathrm{ml} / \mathrm{min}$, retention time: $\mathrm{t}_{\text {major }}=12.5, \mathrm{t}_{\text {minor }}=10.5,74 \%$ ee .

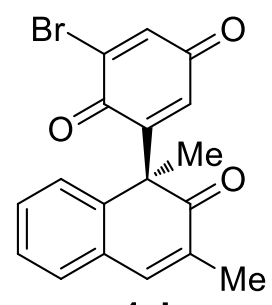

$4 q^{\prime}$

(S)-2-bromo-6-(1,3-dimethyl-2-oxo-1,2-dihydronaphthalen-1-yl)cyclohexa-2,5-diene-1,4-dion e, 4q'.

Using $10 \mathrm{~mol} \%$ cat. $3 \mathrm{~g}$ at $\mathrm{rt}$ with $22 \mathrm{~h}, 39 \mathrm{mg}$ ( $55 \%$ yield) of the pure product was obtained by silica gel column chromatography (petroleum/ethyl acetate $=20: 1-15: 1)$ as a yellow solid; ${ }^{1} \mathbf{H}$ $\operatorname{NMR}\left(300 \mathrm{MHz}, \mathrm{CDCl}_{3}\right) \delta 7.40(\mathrm{~s}, 1 \mathrm{H}), 7.35(\mathrm{dd}, J=7.2,1.7 \mathrm{~Hz}, 1 \mathrm{H}), 7.31-7.22(\mathrm{~m}, 2 \mathrm{H}), 7.21$ (s, 1H), $7.12(\mathrm{~s}, 1 \mathrm{H}), 6.94-6.82(\mathrm{~m}, 1 \mathrm{H}), 2.05(\mathrm{~d}, J=1.2 \mathrm{~Hz}, 3 \mathrm{H}), 1.50(\mathrm{~s}, 3 \mathrm{H}) .{ }^{13} \mathbf{C}$ NMR $(75$ $\left.\mathrm{MHz}, \mathrm{CDCl}_{3}\right) \delta 200.4,183.1,179.5,152.4,143.3,141.5,138.1,137.3,133.2,131.4,129.4,129.1$, 129.0, 127.5, 125.2, 53.2, 26.6, 15.9. IR: 3301， 2925, 1743, 1666, 1595, 1458, 1375, 1259, 1197 , $1001,912,738 \mathrm{~cm}^{-1} ;[\boldsymbol{\alpha}]_{\mathbf{D}}{ }^{\mathbf{r t}}=24^{\circ}\left(\mathrm{c}=1.00, \mathrm{CHCl}_{3}\right) ; \mathrm{HRMS}(\mathrm{ESI}): \mathrm{C}_{18} \mathrm{H}_{13} \mathrm{ClO}_{3}+\mathrm{Na}$, Calc: 378.9940, Found: 378.9956; HPLC: DAICEL CHIRALCEL IA, Hexane/iPrOH = 8/2, flow rate = $1.0 \mathrm{ml} / \mathrm{min}$, retention time: $\mathrm{t}_{\text {major }}=11.0, \mathrm{t}_{\text {minor }}=13.0,45 \%$ ee.<smiles>CC1=Cc2ccccc2[C@@](C)(C2=CC(=O)C=C(Cl)C2=O)C1=O</smiles>

(R)-2-chloro-6-(1,3-dimethyl-2-oxo-1,2-dihydronaphthalen-1-yl)cyclohexa-2,5-diene-1,4-dion e, $4 \mathbf{r}$

Using $10 \mathrm{~mol} \%$ cat. $3 \mathrm{~g}$ at $\mathrm{rt}$ with $40 \mathrm{~h}, 32 \mathrm{mg}$ ( $51 \%$ yield) of the pure product was obtained by silica gel column chromatography (petroleum/ethyl acetate $=20: 1-15: 1$ ) as a yellow solid; ${ }^{1} \mathbf{H}$ 
NMR $\left(300 \mathrm{MHz}, \mathrm{CDCl}_{3}\right) \delta 7.40(\mathrm{~s}, 1 \mathrm{H}), 7.39-7.32(\mathrm{~m}, 1 \mathrm{H}), 7.31-7.18(\mathrm{~m}, 2 \mathrm{H}), 7.17(\mathrm{~s}, 1 \mathrm{H})$, $6.96-6.86(\mathrm{~m}, 1 \mathrm{H}), 6.84(\mathrm{~s}, 1 \mathrm{H}), 2.05(\mathrm{~d}, J=1.1 \mathrm{~Hz}, 3 \mathrm{H}), 1.51(\mathrm{~s}, 3 \mathrm{H}) .{ }^{13} \mathbf{C}$ NMR $(75 \mathrm{MHz}$, $\left.\mathrm{CDCl}_{3}\right) \delta 200.4,183.5,179.6,152.5,143.9,143.3,141.5,133.8,133.5,131.4,129.5,129.2,129.0$, 127.5, 125.2, 53.2, 26.6, 16.0. IR: 3394, 2924, 1656, 1593, 1457, 1379, 1261, 1027, 808, $607 \mathrm{~cm}^{-1}$; $[\boldsymbol{\alpha}]_{\mathbf{D}}{ }^{\mathbf{r t}}=-33^{\circ}\left(\mathrm{c}=1.00, \mathrm{CHCl}_{3}\right) ; \mathrm{HRMS}(\mathrm{ESI}): \mathrm{C}_{18} \mathrm{H}_{13} \mathrm{ClO}_{3}+\mathrm{H}$, Calc: 313.0626 , Found: 313.0624; HPLC: DAICEL CHIRALCEL IC, Hexane/iPrOH $=9 / 1$, flow rate $=1.0 \mathrm{ml} / \mathrm{min}$, retention time: $\mathrm{t}_{\text {major }}=12.5, \mathrm{t}_{\text {minor }}=13.4,89 \%$ ee.<smiles>CC1=CC2C(C)=Cc3ccccc3C2(C2=CC(=O)C=C(Cl)C2=O)C1=O</smiles>

2-chloro-6-(1,3-dimethyl-2-oxo-1,2-dihydronaphthalen-1-yl)cyclohexa-2,5-diene-1,4-dione, $4 \mathbf{r}^{\prime}$

Using $10 \mathrm{~mol} \%$ cat. $3 \mathrm{~g}$ at $\mathrm{rt}$ with $22 \mathrm{~h}, 40 \mathrm{mg}$ (64\% yield) of the pure product was obtained by silica gel column chromatography (petroleum/ethyl acetate $=20: 1-10: 1$ ) as a yellow solid; ${ }^{1} \mathbf{H}$ NMR $\left(300 \mathrm{MHz}, \mathrm{CDCl}_{3}\right) \delta 7.40(\mathrm{~s}, 1 \mathrm{H}), 7.39-7.32(\mathrm{~m}, 1 \mathrm{H}), 7.31-7.18(\mathrm{~m}, 2 \mathrm{H}), 7.17(\mathrm{~s}, 1 \mathrm{H})$, $6.96-6.86(\mathrm{~m}, 1 \mathrm{H}), 6.84(\mathrm{~s}, 1 \mathrm{H}), 2.05(\mathrm{~d}, J=1.1 \mathrm{~Hz}, 3 \mathrm{H}), 1.51$ (s, 3H). ${ }^{13} \mathbf{C}$ NMR $(75 \mathrm{MHz}$, $\left.\mathrm{CDCl}_{3}\right) \delta 200.4,183.5,179.6,152.5,143.9,143.3,141.5,133.8,133.5,131.4,129.5,129.2,129.0$, 127.5, 125.2, 53.2, 26.6, 16.0. IR: 3394, 2924, 1656, 1593, 1457, 1379, 1261, 1027, 808, $607 \mathrm{~cm}^{-1}$; HRMS (ESI): $\mathrm{C}_{18} \mathrm{H}_{13} \mathrm{ClO}_{3}+\mathrm{H}$, Calc: 313.0626 , Found: 313.0624 .<smiles>CC1=CC2C(C)=Cc3ccccc3[C@]2(C2=CC(=O)C(Cl)=CC2=O)C1=O</smiles>

4s

(S)-2-chloro-5-(1,3-dimethyl-2-oxo-1,2-dihydronaphthalen-1-yl)cyclohexa-2,5-diene-1,4-dion 
e, $4 \mathrm{~s}$.

Using $10 \mathrm{~mol} \%$ cat. $3 \mathrm{~g}$ at $\mathrm{rt}$ with $30 \mathrm{~h}, 26 \mathrm{mg}$ ( $41 \%$ yield) of the pure product was obtained by silica gel column chromatography (petroleum/ethyl acetate $=20: 1-15: 1$ ) as a yellow solid; ${ }^{1} \mathbf{H}$ NMR $\left(300 \mathrm{MHz}, \mathrm{CDCl}_{3}\right) \delta 7.41(\mathrm{~s}, 1 \mathrm{H}), 7.36(\mathrm{dd}, J=7.3,1.6 \mathrm{~Hz}, 1 \mathrm{H}), 7.31-7.18(\mathrm{~m}, 2 \mathrm{H}), 7.05$ (d, $J=2.4 \mathrm{~Hz}, 1 \mathrm{H}), 7.00$ (d, $J=2.4 \mathrm{~Hz}, 1 \mathrm{H}), 6.94-6.86$ (m, 1H), 2.06 (d, $J=1.2 \mathrm{~Hz}, 3 \mathrm{H}), 1.51$ (s, 3H). ${ }^{13}$ C NMR (75 MHz, $\left.\mathrm{CDCl}_{3}\right) \delta 200.3,184.9,178.3,151.8,144.2,143.1,141.6,134.2,133.4$, 131.5, 129.5, 129.2, 129.1, 127.6, 125.1, 53.6, 26.9, 15.9. IR: 3304, 3054, 2926, 1662, 1594, 1458, $1422,1265,1014,896,739 \mathrm{~cm}^{-1} ;[\boldsymbol{\alpha}]_{\mathbf{D}}{ }^{\mathbf{r t}}=7^{\circ}\left(\mathrm{c}=1.00, \mathrm{CHCl}_{3}\right)$; HRMS (ESI): $\mathrm{C}_{18} \mathrm{H}_{13} \mathrm{ClO}_{3}+\mathrm{H}, \mathrm{Calc}$ : 313.0626, Found: 313.0640; HPLC: DAICEL CHIRALCEL IA, Hexane/iPrOH $=8 / 2$, flow rate $=$ $1.0 \mathrm{ml} / \mathrm{min}$, retention time: $\mathrm{t}_{\text {major }}=7.6, \mathrm{t}_{\mathrm{minor}}=9.3,18 \%$ ee.

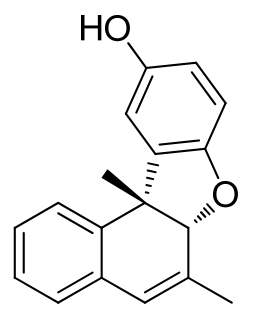

5

(6aR,11bR)-6,11b-dimethyl-6a,11b-dihydronaphtho[2,1-b]benzofuran-10-ol, 5

The solvent was removed under vacuum and residue was chromatographed on silica gel (petroleum ether/AcOEt 15:1) to provide the pure desired products $40 \mathrm{mg}$ (75\% yield) as a white solid; ${ }^{1} \mathbf{H}$ NMR (300 MHz, $\left.\mathrm{CDCl}_{3}\right) \delta 7.49-7.38(\mathrm{~m}, 1 \mathrm{H}), 7.23-7.08$ (m, 2H), 7.04 (dd, $J=$ $7.2,1.6 \mathrm{~Hz}, 1 \mathrm{H}), 6.86(\mathrm{~d}, J=2.6 \mathrm{~Hz}, 1 \mathrm{H}), 6.66(\mathrm{~d}, J=8.4 \mathrm{~Hz}, 1 \mathrm{H}), 6.56(\mathrm{dd}, J=8.5,2.6 \mathrm{~Hz}, 1 \mathrm{H})$, $6.42(\mathrm{~s}, 1 \mathrm{H}), 4.82(\mathrm{~s}, 1 \mathrm{H}), 4.58(\mathrm{~s}, 1 \mathrm{H}), 2.09(\mathrm{~d}, J=0.8 \mathrm{~Hz}, 3 \mathrm{H}), 1.58(\mathrm{~s}, 3 \mathrm{H}),{ }^{13} \mathbf{C}$ NMR $(75 \mathrm{MHz}$, $\left.\mathrm{CDCl}_{3}\right) \delta 152.3,149.8,137.2,136.3,131.0,130.7,127.6,127.1,126.9,126.9,126.1,114.3,111.6$, 109.7, 90.8, 47.5, 27.9, 21.1; IR: 3395, 2923, 1656, 1602, 1489, 1376, 1268, 1192, 1068, 947, 812, $753 \mathrm{~cm}^{-1} ;[\boldsymbol{\alpha}]_{\mathbf{D}}{ }^{\mathbf{r t}}=117^{\circ}\left(\mathrm{c}=1.00, \mathrm{CHCl}_{3}\right)$; HRMS (ESI): $\mathrm{C}_{18} \mathrm{H}_{16} \mathrm{O}_{2}+\mathrm{H}$, Calc: 265.1223 , Found: 265.1225; HPLC: DAICEL CHIRALCEL IA, Hexane $/ \mathrm{EtOH}=95 / 5$, flow rate $=1.0 \mathrm{ml} / \mathrm{min}$, retention time: $\mathrm{t}_{\text {major }}=9.7, \mathrm{t}_{\text {minor }}=13.7,98 \%$ ee. 


\section{Determination of the absolute configuration 4.}

\section{X-ray Structure of 4a:}
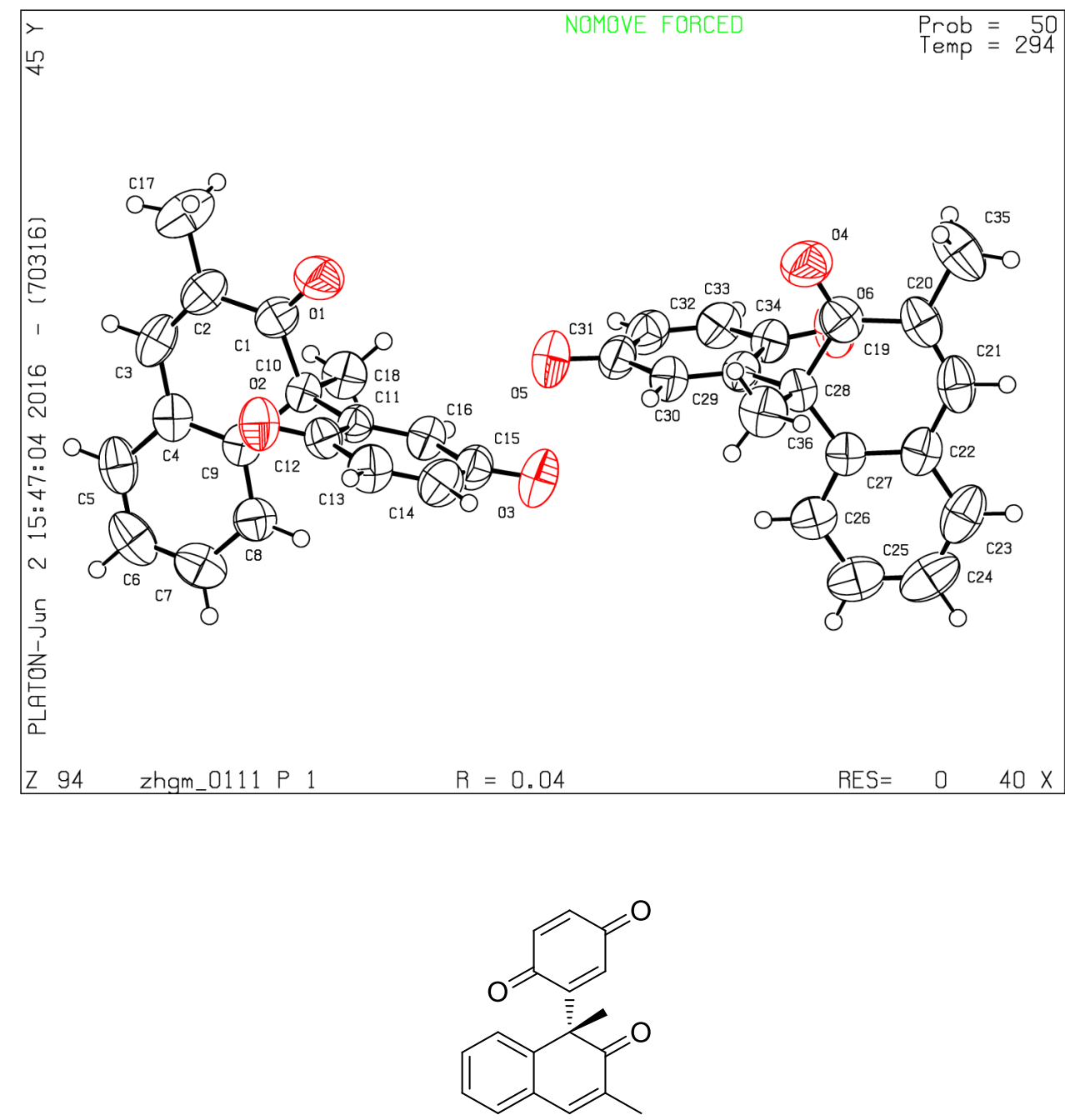

$4 a$

Bond precision:

$\mathrm{C}-\mathrm{C}=0.0069 \mathrm{~A}$

Wavelength $=1.54184$

Cell:

$$
\begin{array}{ll}
a=7.6607(7) & b=9.2315(7) \\
\text { alpha=72.019(7) } & \text { beta=79.887(7) }
\end{array}
$$$$
\mathrm{c}=11.1270(8)
$$

gamma $=74.428(7)$

Temperature: $294 \mathrm{~K}$

Calculated

$717.29(11)$

Space group

Hall group
P 1

P 1
Reported

$717.29(11)$

P 1

P 1 


\begin{tabular}{|c|c|c|}
\hline Moiety formula & C18 H14 O3 & C18 H14 O3 \\
\hline Sum formula & C18 H14 O3 & C18 H14 O3 \\
\hline $\mathrm{Mr}$ & 278.29 & 278.29 \\
\hline Dx,g cm-3 & 1.288 & 1.289 \\
\hline $\mathrm{Z}$ & 2 & 2 \\
\hline $\mathrm{Mu}(\mathrm{mm}-1)$ & 0.708 & 0.708 \\
\hline F000 & 292.0 & 292.0 \\
\hline F000' & 292.91 & \\
\hline $\mathrm{h}, \mathrm{k}, \operatorname{lmax}$ & $9,11,13$ & $9,11,13$ \\
\hline Nref & $5446[2723]$ & 4653 \\
\hline Tmin,Tmax & $0.887,0.906$ & $0.869,1.000$ \\
\hline Tmin' & 0.887 & \\
\hline \multicolumn{3}{|c|}{ Correction method $=\#$ Reported $\mathrm{T}$ Limits: $\mathrm{Tmin}=0.869 \mathrm{Tmax}=1.000$ AbsCorr $=$} \\
\hline \multicolumn{3}{|l|}{ MULTI-SCAN } \\
\hline \multicolumn{2}{|c|}{ Data completeness $=1.71 / 0.85$} & $\operatorname{Theta}(\max )=69.915$ \\
\hline \multicolumn{2}{|c|}{$\mathrm{R}($ reflections $)=0.0429(3320)$} & $\mathrm{wR} 2($ reflections $)=0.1185(4653)$ \\
\hline$S=1.056$ & Npar $=383$ & \\
\hline
\end{tabular}


8. HPLC Analytic Conditions of 4 and 5:

All products are separated by using DAICEL CHIRALCEL column.

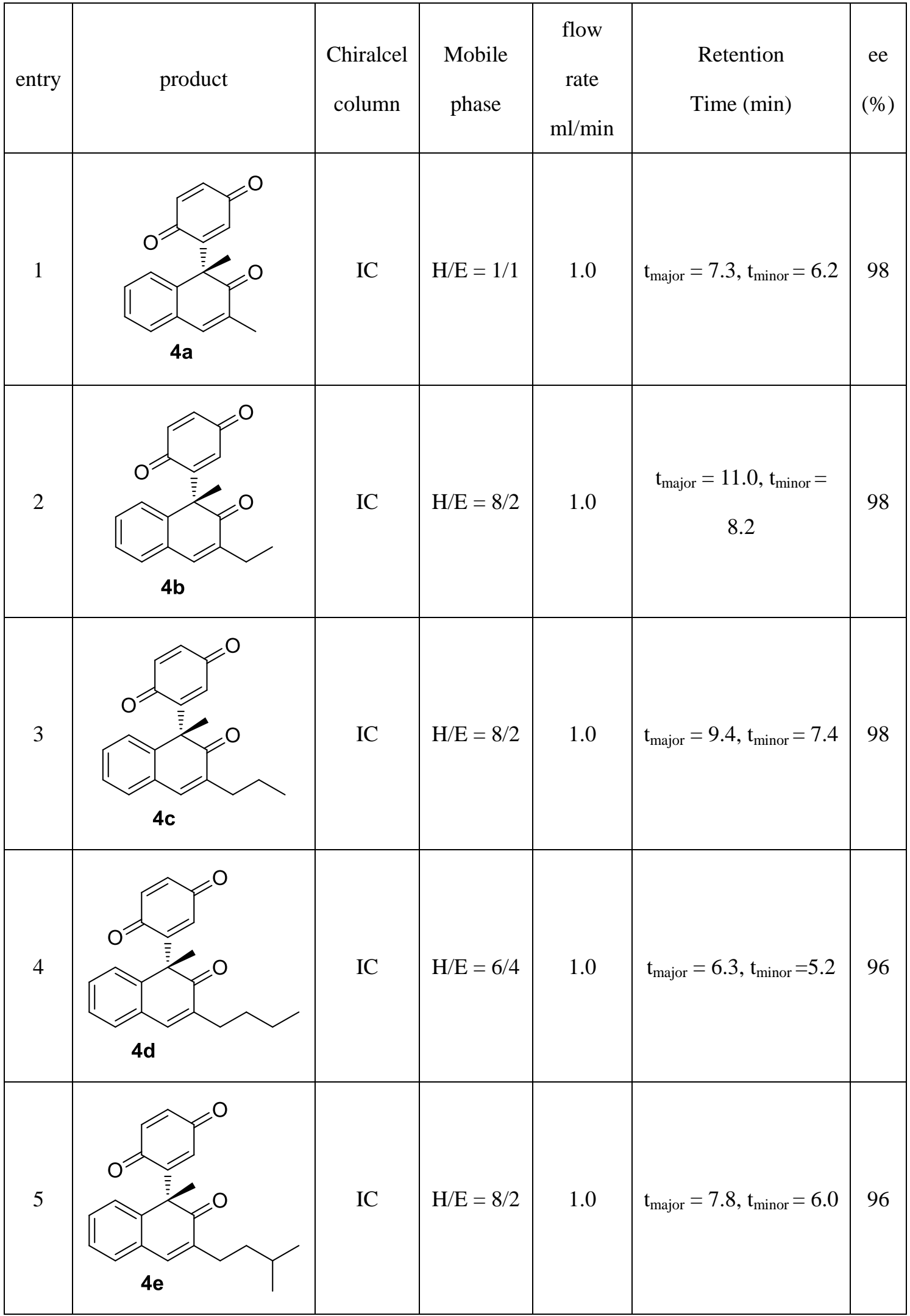




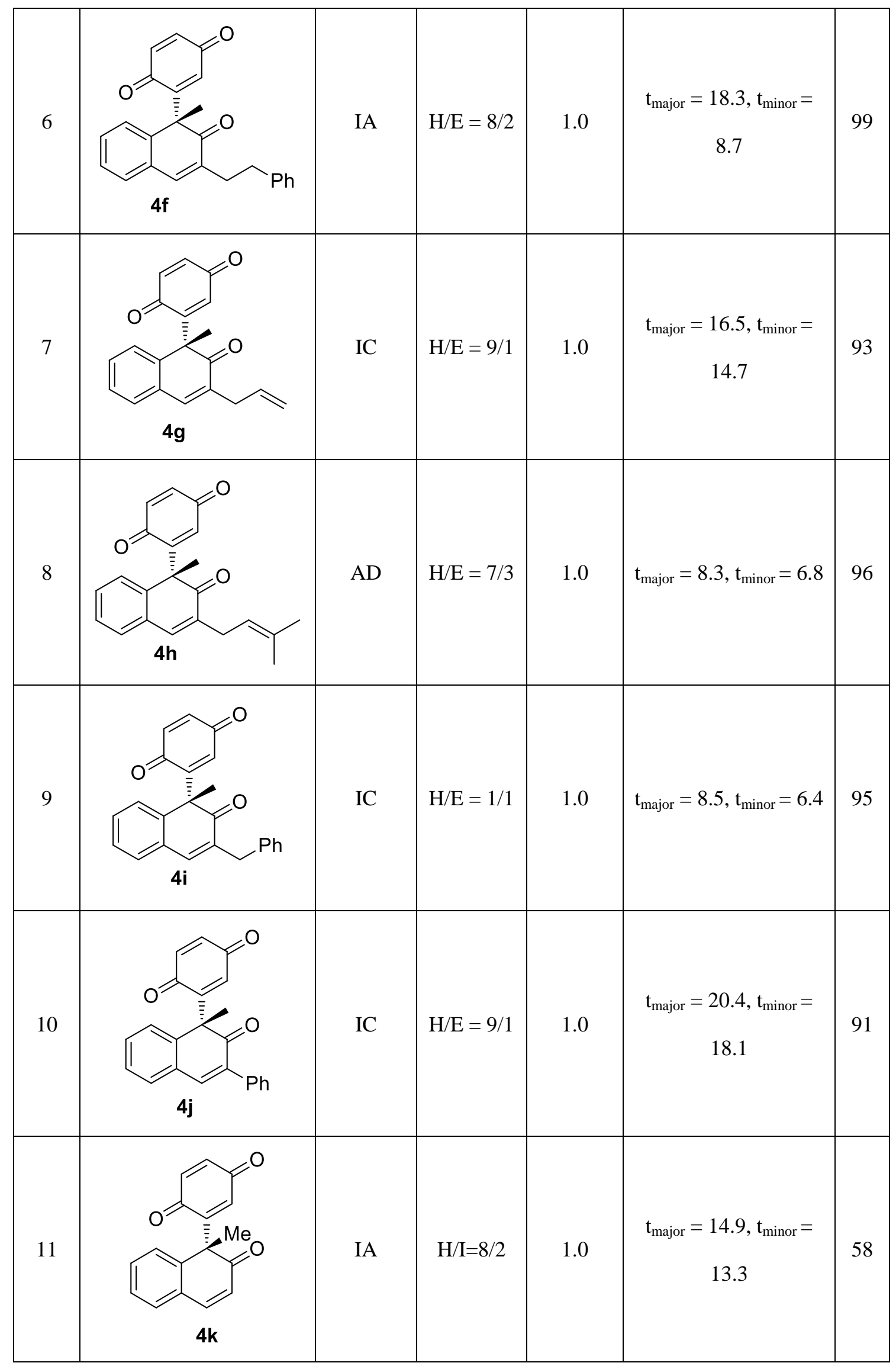




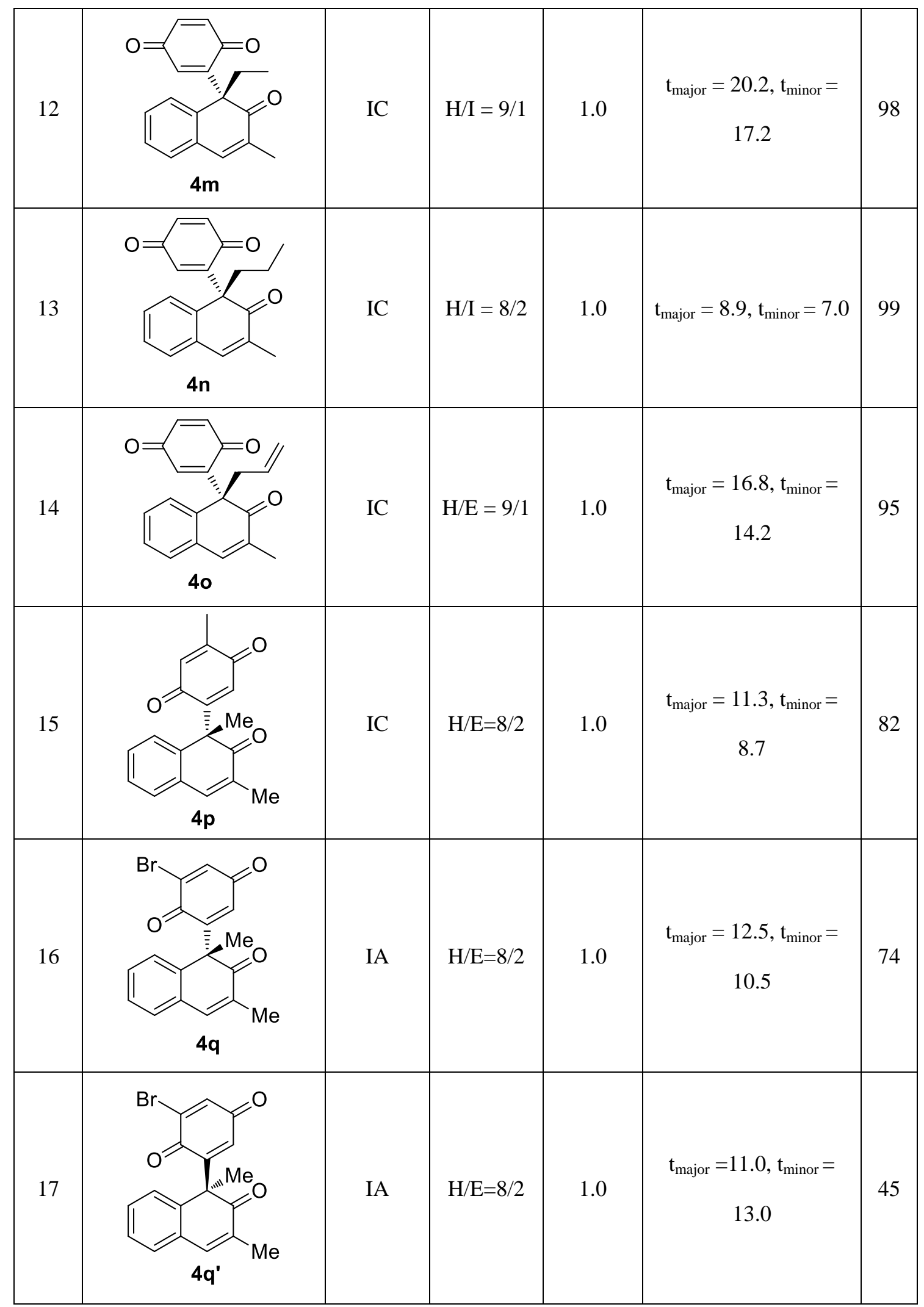




\begin{tabular}{|l|l|l|l|l|l|}
\hline 18 & IC & $\mathrm{H} / \mathrm{E}=9 / 1$ & 1.0 & $\mathrm{t}_{\text {major }}=12.5, \mathrm{t}_{\text {minor }}=$ & \\
\hline 19 & & & & & \\
\hline
\end{tabular}




\section{Copies of HPLC spectra for 4 and 5.}

(R)-2-(1,3-dimethyl-2-oxo-1,2-dihydronaphthalen-1-yl)cyclohexa-2,5-diene-1,4-dione

4a (Table 2)

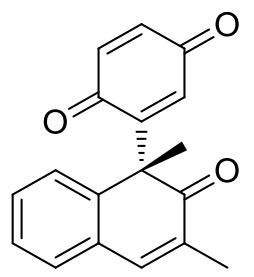

$4 a$

Chiralpak IC column, hexane/EtOH (1:1), flow rate $1.0 \mathrm{~mL} / \mathrm{min}$

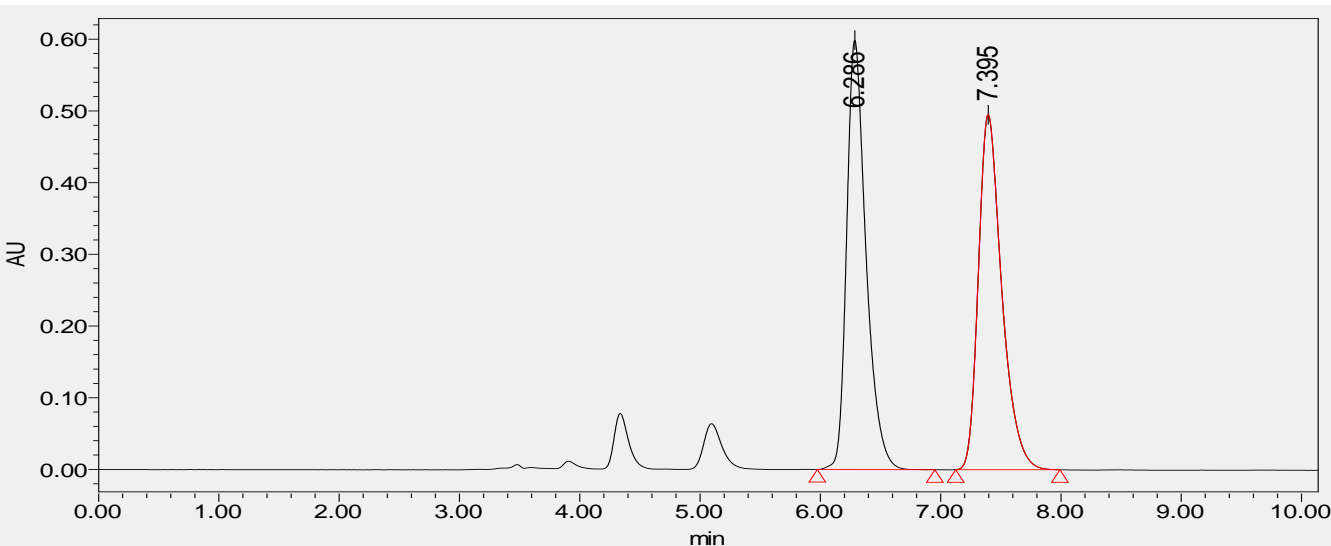

\begin{tabular}{|c|c|c|c|c|c|}
\hline & Retention time & Area & $\%$ Area & Height & Integral type \\
\hline 1 & 6.286 & 6703713 & 50.03 & 598924 & $\mathrm{bb}$ \\
\hline 2 & 7.395 & 6695800 & 49.97 & 495218 & $\mathrm{bb}$ \\
\hline
\end{tabular}

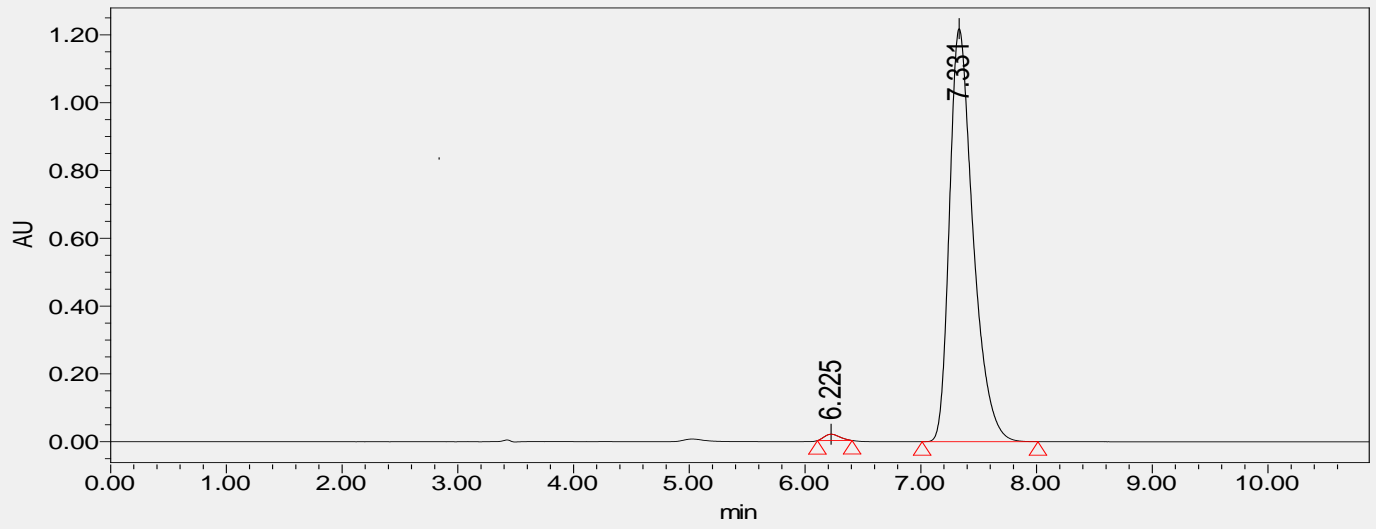

\begin{tabular}{|c|c|c|c|c|c|}
\hline & Retention time & Area & $\%$ Area & Height & Integral type \\
\hline 1 & 6.225 & 172225 & 0.99 & 18739 & $\mathrm{bb}$ \\
\hline 2 & 7.331 & 17223772 & 99.01 & 1218144 & $\mathrm{bb}$ \\
\hline
\end{tabular}


(R)-2-(3-ethyl-1-methyl-2-oxo-1,2-dihydronaphthalen-1-yl)cyclohexa-2,5-diene-1,4-dione

4b (Table 2)

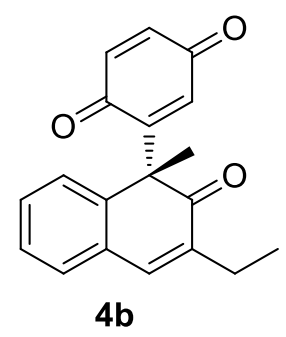

Chiralpak IC column, hexane/EtOH (8:2), flow rate $1.0 \mathrm{~mL} / \mathrm{min}$

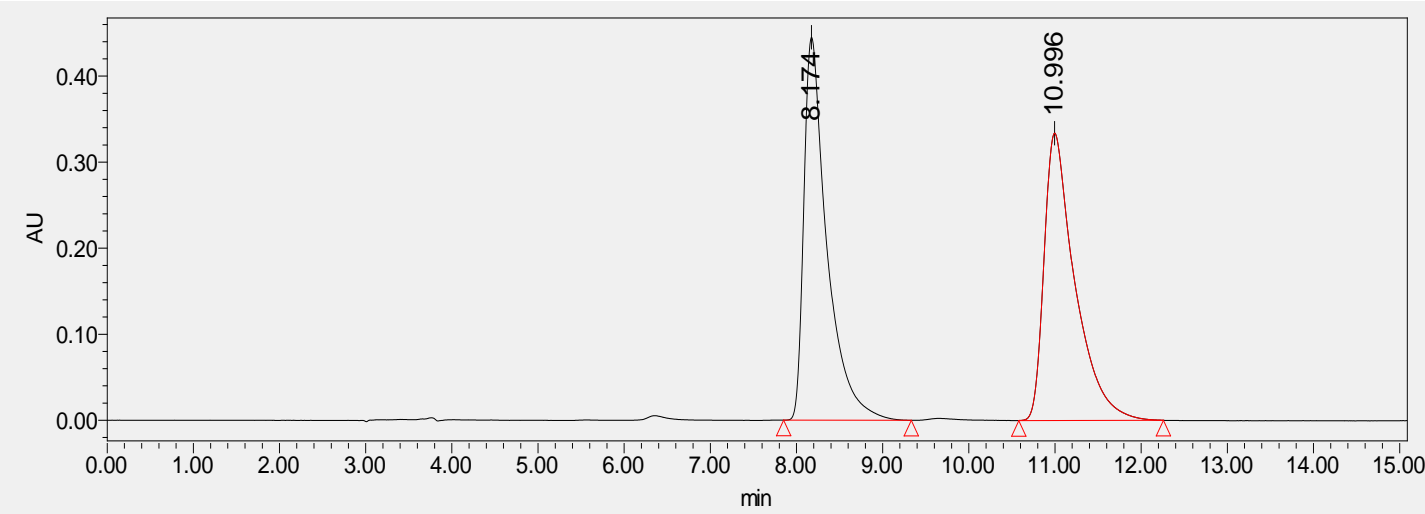

\begin{tabular}{|c|c|c|c|c|c|}
\hline & Retention time & Area & $\%$ Area & Height & Integral type \\
\hline 1 & 8.174 & 8266822 & 49.88 & 444993 & bb \\
\hline 2 & 10.996 & 8307541 & 50.12 & 333850 & bb \\
\hline
\end{tabular}

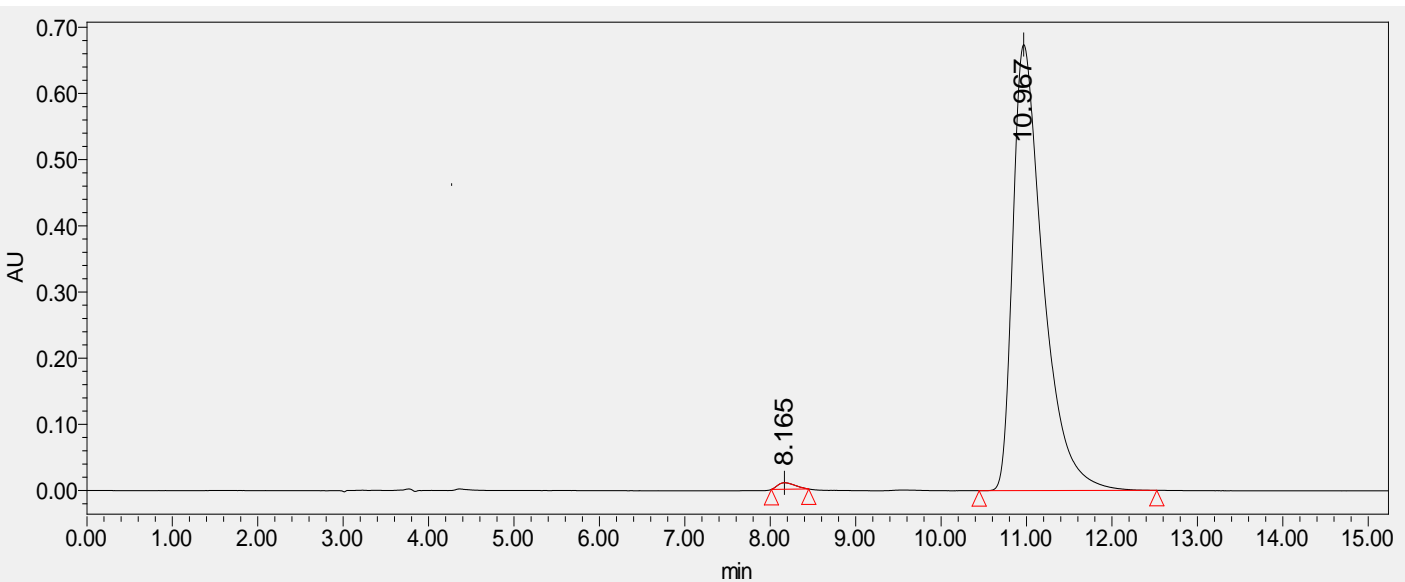

\begin{tabular}{|c|c|c|c|c|c|}
\hline & Retention time & Area & $\%$ Area & Height & Integral type \\
\hline 1 & 8.165 & 139575 & 0.84 & 9976 & $\mathrm{bb}$ \\
\hline 2 & 10.967 & 16557871 & 99.16 & 673993 & $\mathrm{bb}$ \\
\hline
\end{tabular}


(R)-2-(1-methyl-2-oxo-3-propyl-1,2-dihydronaphthalen-1-yl)cyclohexa-2,5-diene-1,4-dione

4c (Table 2)

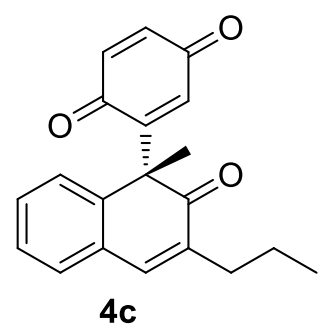

Chiralpak IC column, hexane/EtOH (8:2), flow rate $1.0 \mathrm{~mL} / \mathrm{min}$

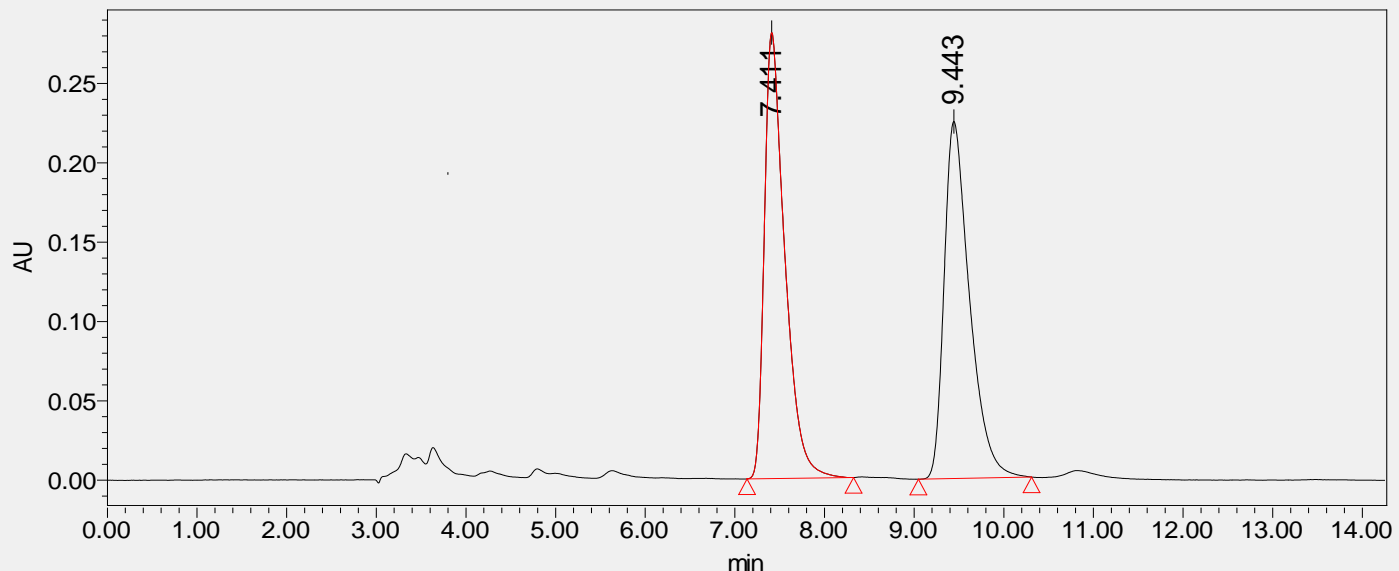

\begin{tabular}{|c|c|c|c|c|c|}
\hline & Retention time & Area & $\%$ Area & Height & Integral type \\
\hline 1 & 7.411 & 4458380 & 50.14 & 281075 & $\mathrm{bb}$ \\
\hline 2 & 9.443 & 4433817 & 49.86 & 225089 & $\mathrm{bb}$ \\
\hline
\end{tabular}

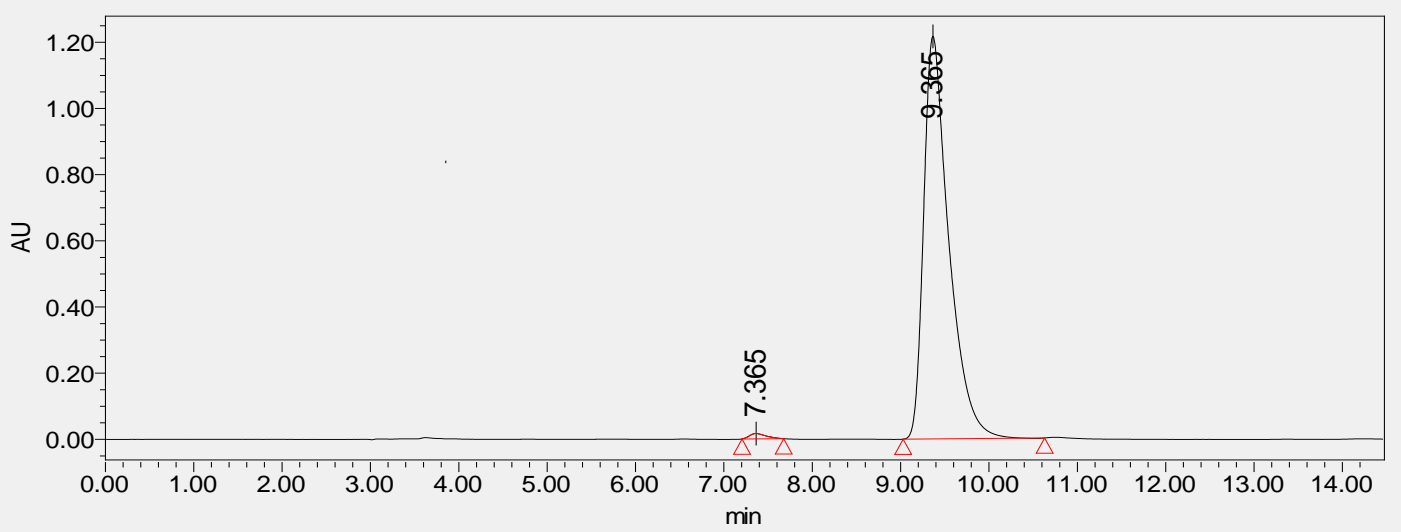

\begin{tabular}{|c|c|c|c|c|c|}
\hline & Retention time & Area & $\%$ Area & Height & Integral type \\
\hline 1 & 7.365 & 212672 & 0.88 & 16211 & $\mathrm{bb}$ \\
\hline 2 & 9.365 & 24087752 & 99.12 & 1216435 & $\mathrm{bb}$ \\
\hline
\end{tabular}


(R)-2-(3-butyl-1-methyl-2-oxo-1,2-dihydronaphthalen-1-yl)cyclohexa-2,5-diene-1,4-dione

4d (Table 2)

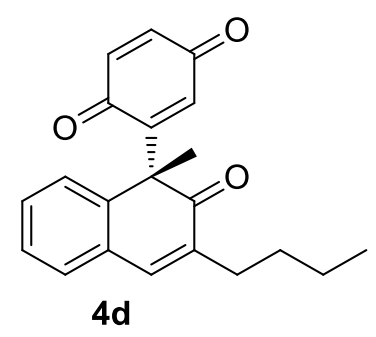

Chiralpak IC column, hexane/EtOH (6:4), flow rate $1.0 \mathrm{~mL} / \mathrm{min}$

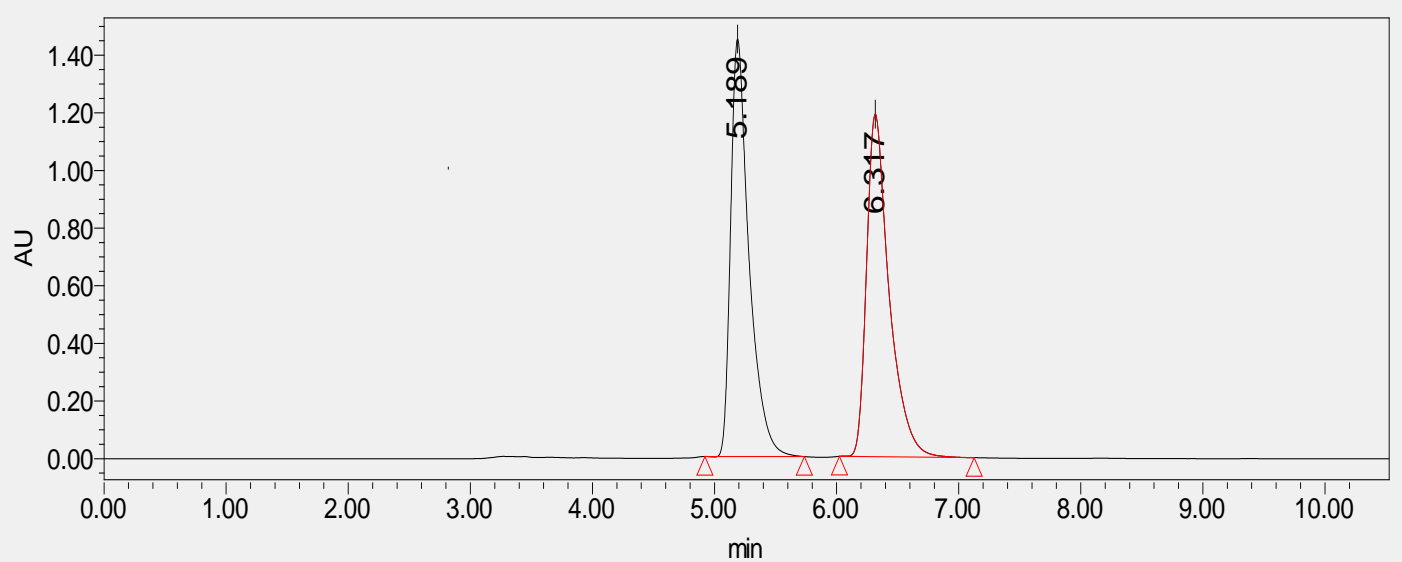

\begin{tabular}{|c|c|c|c|c|c|}
\hline & Retention time & Area & $\%$ Area & Height & Integral type \\
\hline 1 & 5.189 & 15165442 & 49.81 & 1448855 & $\mathrm{bb}$ \\
\hline 2 & 6.317 & 15283257 & 50.19 & 1188509 & $\mathrm{bb}$ \\
\hline
\end{tabular}

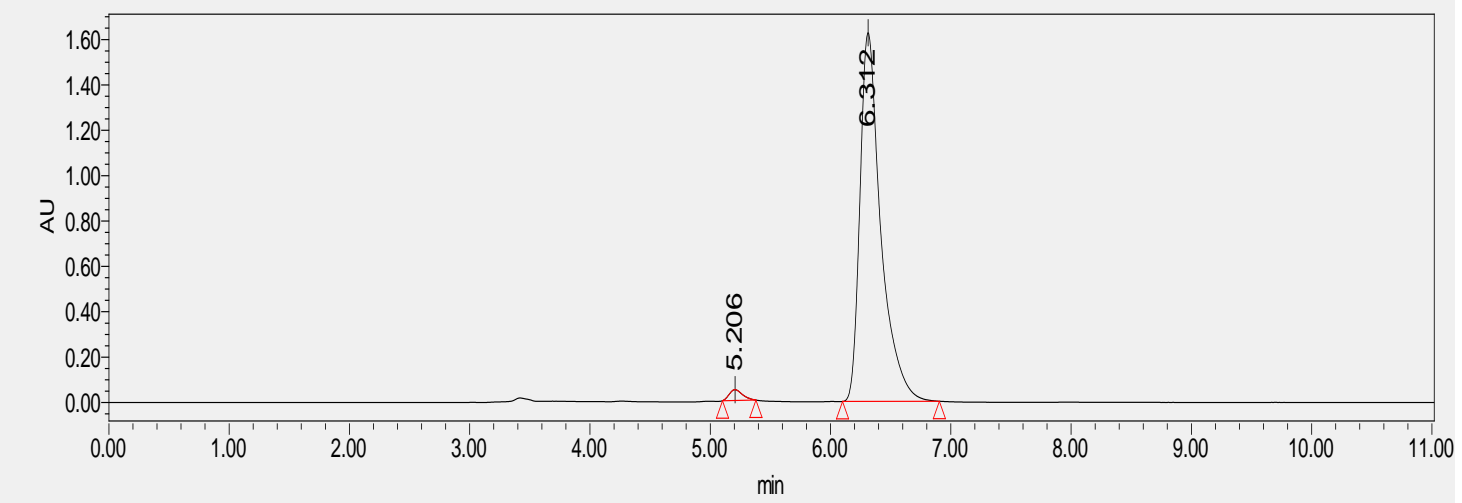

\begin{tabular}{|c|c|c|c|c|c|}
\hline & Retention time & Area & $\%$ Area & Height & Integral type \\
\hline 1 & 5.206 & 364486 & 1.83 & 47728 & $\mathrm{bb}$ \\
\hline 2 & 6.312 & 19567242 & 98.17 & 1625305 & $\mathrm{bb}$ \\
\hline
\end{tabular}


(R)-2-(3-isopentyl-1-methyl-2-oxo-1,2-dihydronaphthalen-1-yl)cyclohexa-2,5-diene-1,4-dione

4e (Table 2)

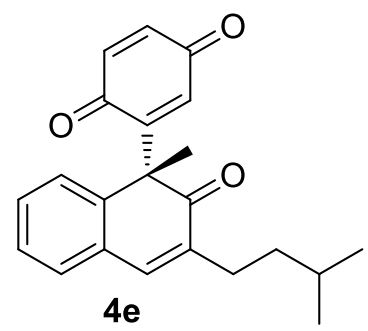

Chiralpak IC column, hexane/EtOH (8:2), flow rate $1.0 \mathrm{~mL} / \mathrm{min}$

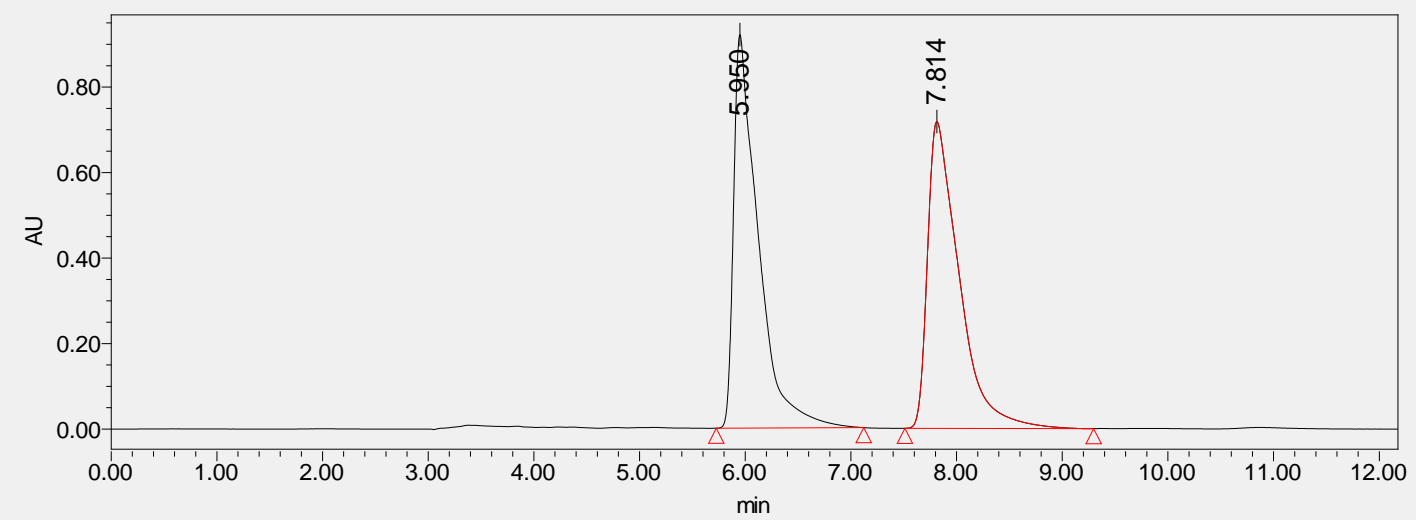

\begin{tabular}{|c|c|c|c|c|c|}
\hline & Retention time & Area & $\%$ Area & Height & Integral type \\
\hline 1 & 5.950 & 14870984 & 49.99 & 920066 & $\mathrm{bb}$ \\
\hline 2 & 7.814 & 14879634 & 50.01 & 717895 & $\mathrm{bb}$ \\
\hline
\end{tabular}

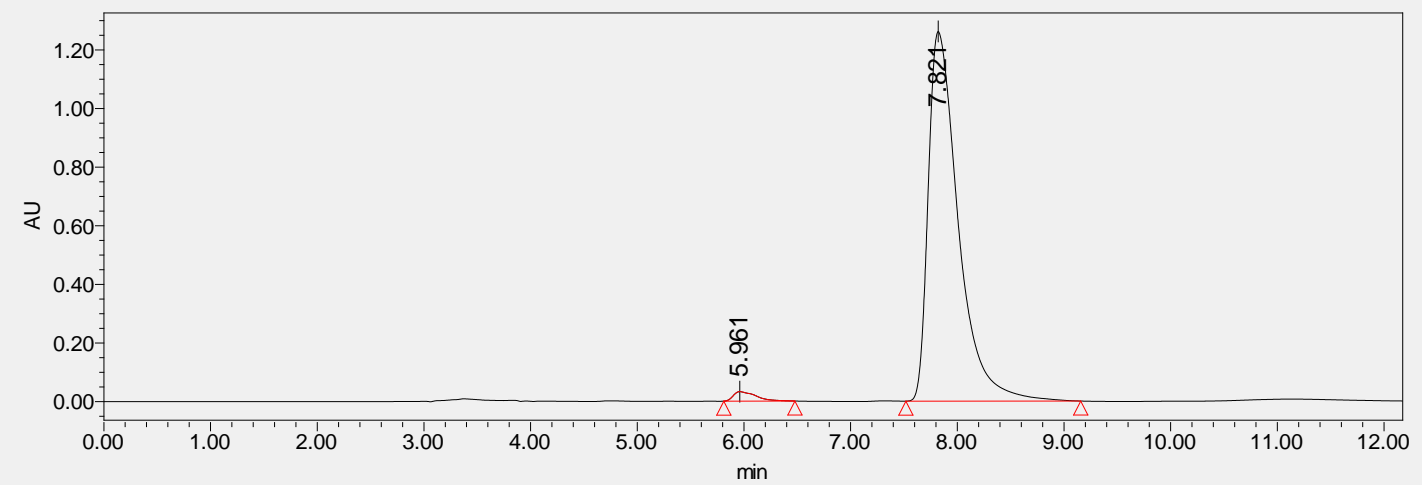

\begin{tabular}{|c|c|c|c|c|c|}
\hline & Retention time & Area & $\%$ Area & Height & Integral type \\
\hline 1 & 5.961 & 475802 & 1.89 & 32527 & $\mathrm{bb}$ \\
\hline 2 & 7.821 & 24686269 & 98.11 & 1261249 & $\mathrm{bb}$ \\
\hline
\end{tabular}


4f (Table 2)

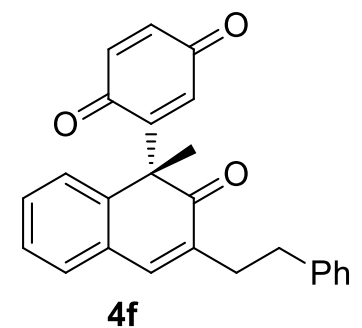

Chiralpak IA column, hexane/EtOH (8:2), flow rate $1.0 \mathrm{~mL} / \mathrm{min}$

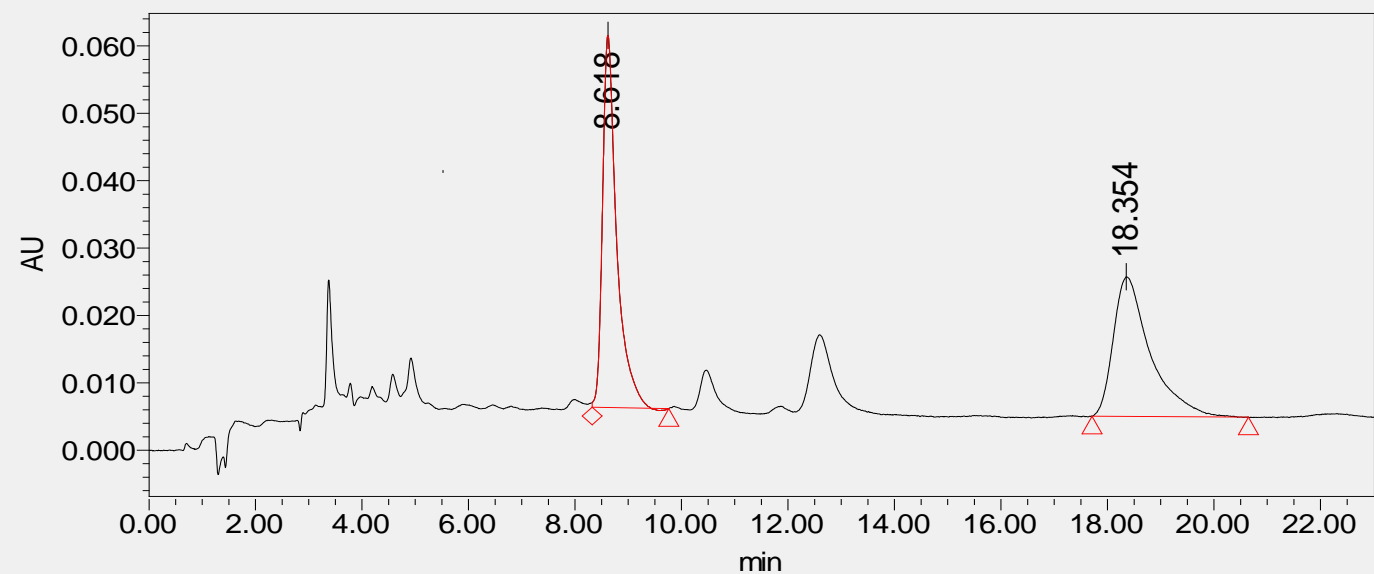

\begin{tabular}{|c|c|c|c|c|c|}
\hline & Retention time & Area & $\%$ Area & Height & Integral type \\
\hline 1 & 8.618 & 1024247 & 50.73 & 55193 & $\mathrm{vb}$ \\
\hline 2 & 18.354 & 994605 & 49.27 & 20725 & $\mathrm{bb}$ \\
\hline
\end{tabular}

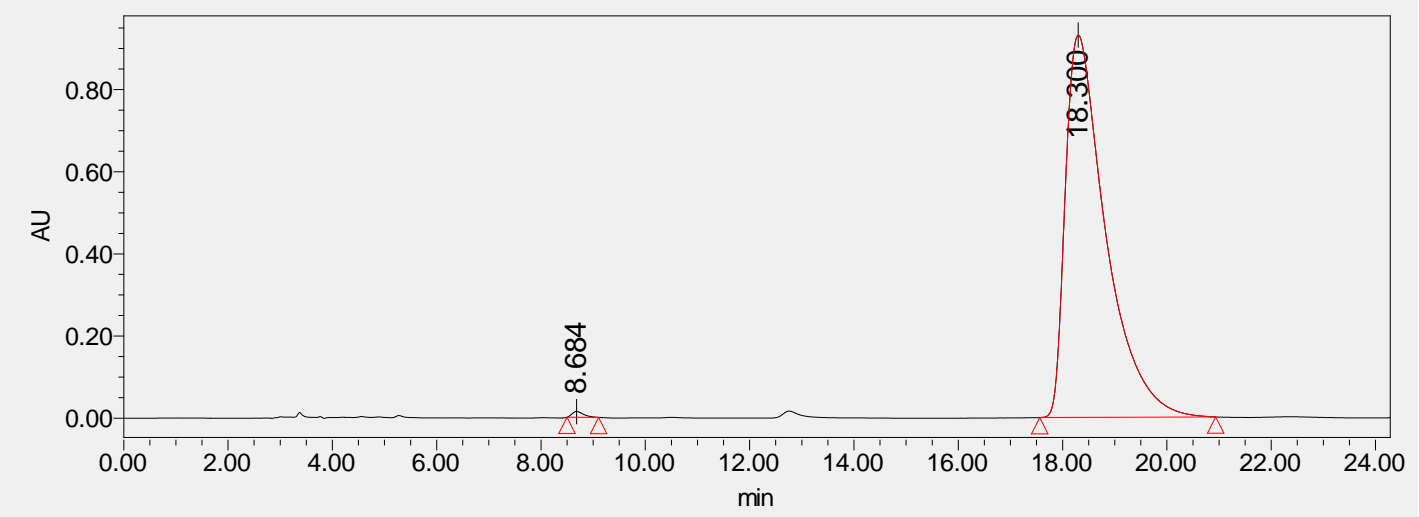

\begin{tabular}{|c|c|c|c|c|c|}
\hline & Retention time & Area & $\%$ Area & Height & Integral type \\
\hline 1 & 8.684 & 219647 & 0.44 & 14178 & $\mathrm{bb}$ \\
\hline 2 & 18.300 & 49967050 & 99.56 & 930727 & $\mathrm{bb}$ \\
\hline
\end{tabular}


(R)-2-(3-allyl-1-methyl-2-oxo-1,2-dihydronaphthalen-1-yl)cyclohexa-2,5-diene-1,4-dione

$4 \mathrm{~g}$ (Table 2)

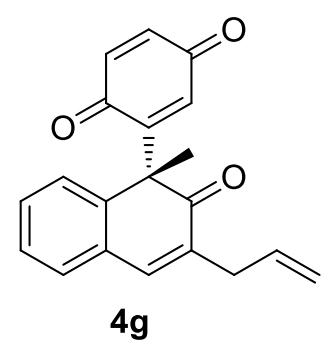

Chiralpak IC column, hexane/EtOH (9:1), flow rate $1.0 \mathrm{~mL} / \mathrm{min}$

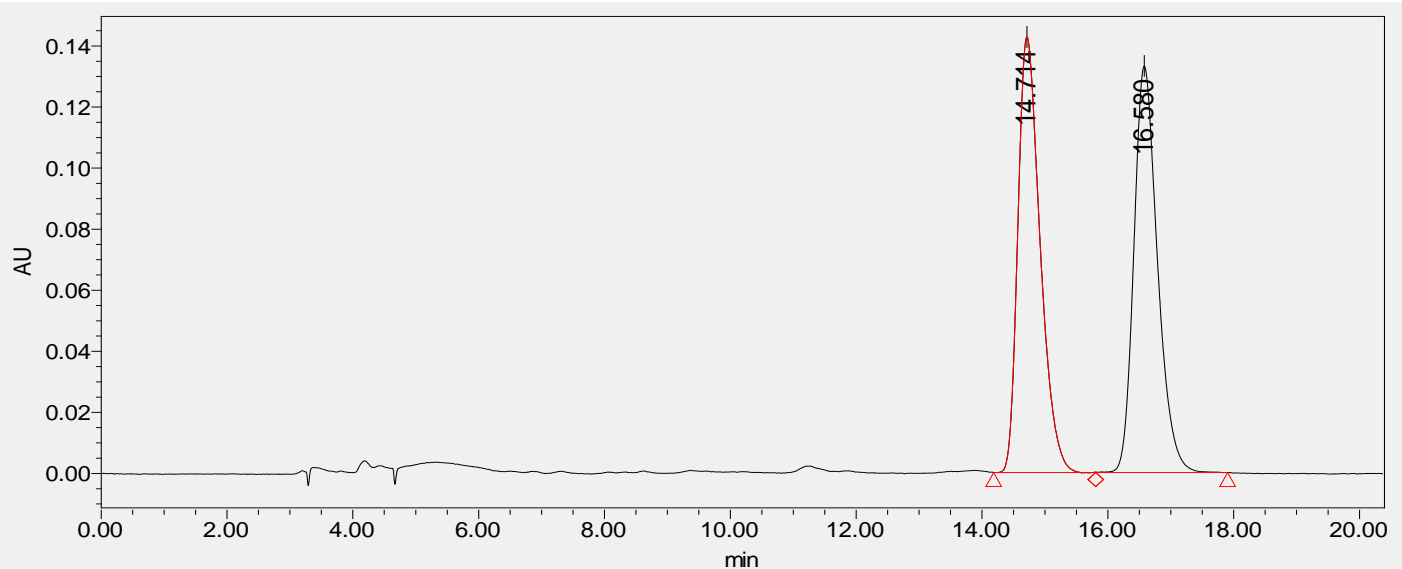

\begin{tabular}{|c|c|c|c|c|c|}
\hline & Retention time & Area & $\%$ Area & Height & Integral type \\
\hline 1 & 14.714 & 3500299 & 50.23 & 142646 & bv \\
\hline 2 & 16.580 & 3467893 & 49.77 & 133081 & $\mathrm{vb}$ \\
\hline
\end{tabular}

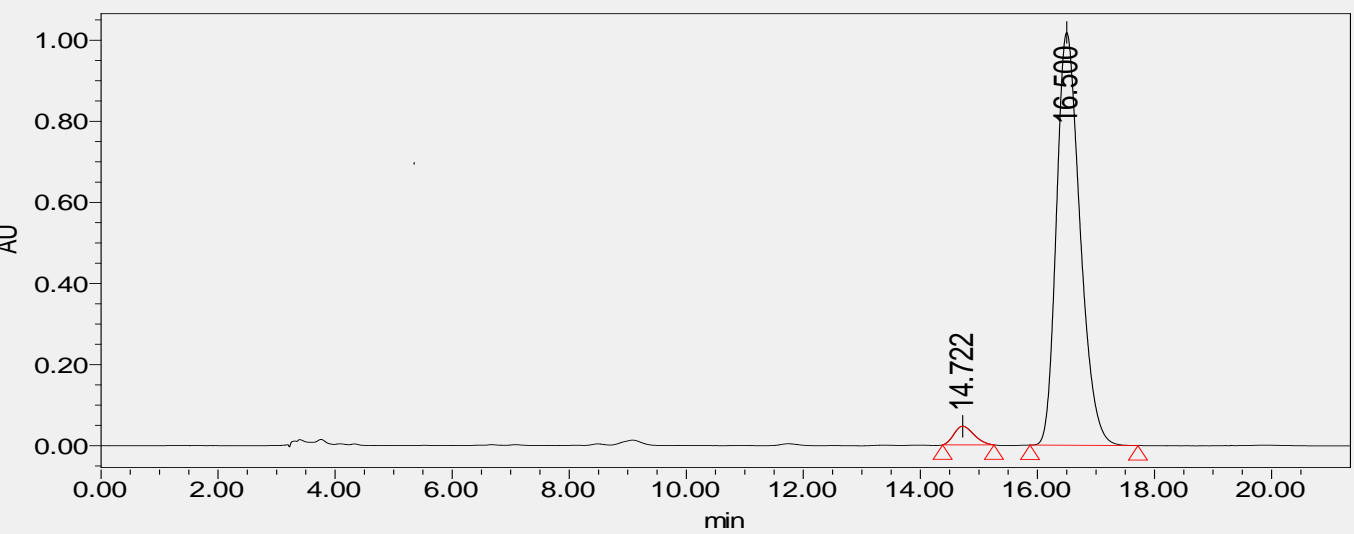

\begin{tabular}{|c|c|c|c|c|c|}
\hline & Retention time & Area & $\%$ Area & Height & Integral type \\
\hline 1 & 14.722 & 1084102 & 3.67 & 45952 & $\mathrm{bb}$ \\
\hline 2 & 16.500 & 28469181 & 96.33 & 1018032 & $\mathrm{bb}$ \\
\hline
\end{tabular}


4h (Table 2)

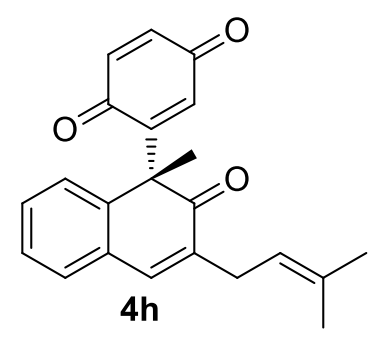

Chiralpak AD column hexane/EtOH (7:3), flow rate $1.0 \mathrm{~mL} / \mathrm{min}$

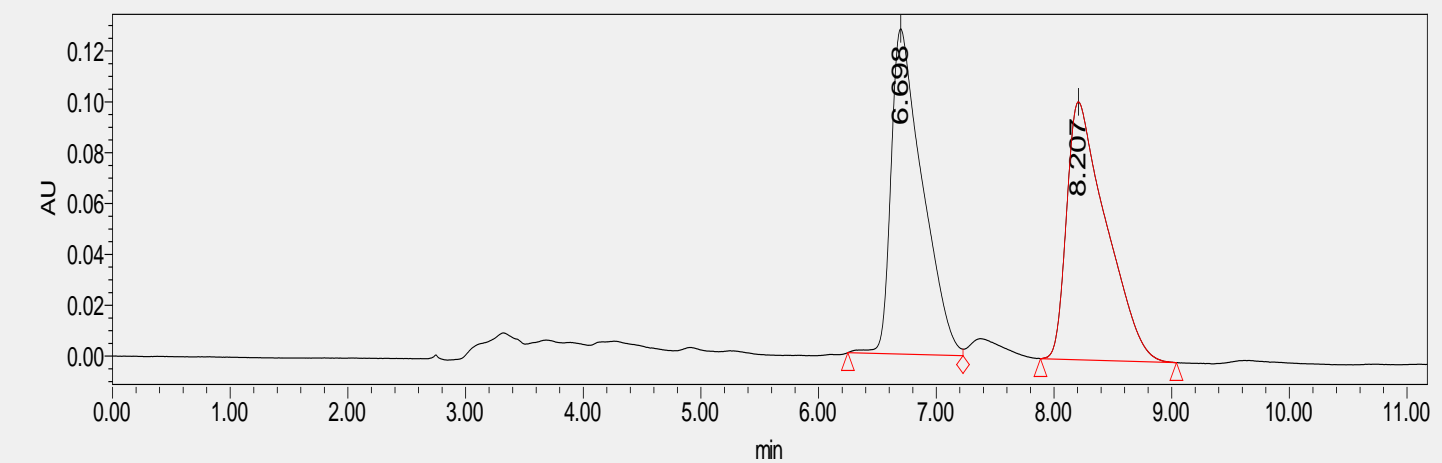

\begin{tabular}{|c|c|c|c|c|c|}
\hline & Retention time & Area & $\%$ Area & Height & Integral type \\
\hline 1 & 6.698 & 2365282 & 49.99 & 127851 & bv \\
\hline 2 & 8.207 & 2366468 & 50.01 & 101432 & $\mathrm{bb}$ \\
\hline
\end{tabular}

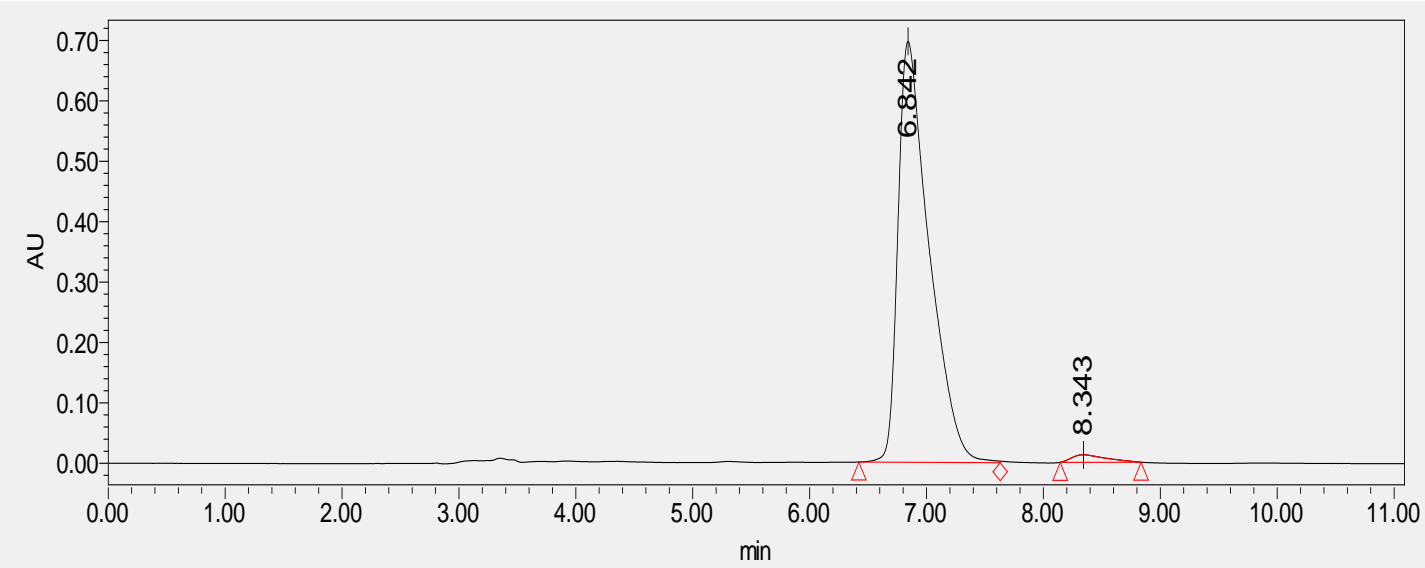

\begin{tabular}{|c|c|c|c|c|c|}
\hline & Retention time & Area & $\%$ Area & Height & Integral type \\
\hline 1 & 6.842 & 12854338 & 98.08 & 696610 & bv \\
\hline 2 & 8.343 & 251814 & 1.92 & 12468 & bb \\
\hline
\end{tabular}


(R)-2-(3-benzyl-1-methyl-2-oxo-1,2-dihydronaphthalen-1-yl)cyclohexa-2,5-diene-1,4-dione

4i (Table 2)

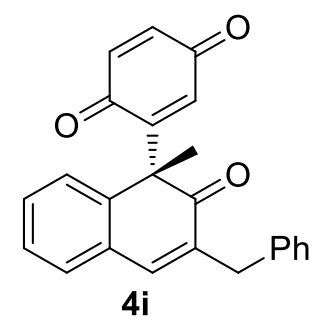

Chiralpak IC column, hexane/EtOH (1:1), flow rate $1.0 \mathrm{~mL} / \mathrm{min}$

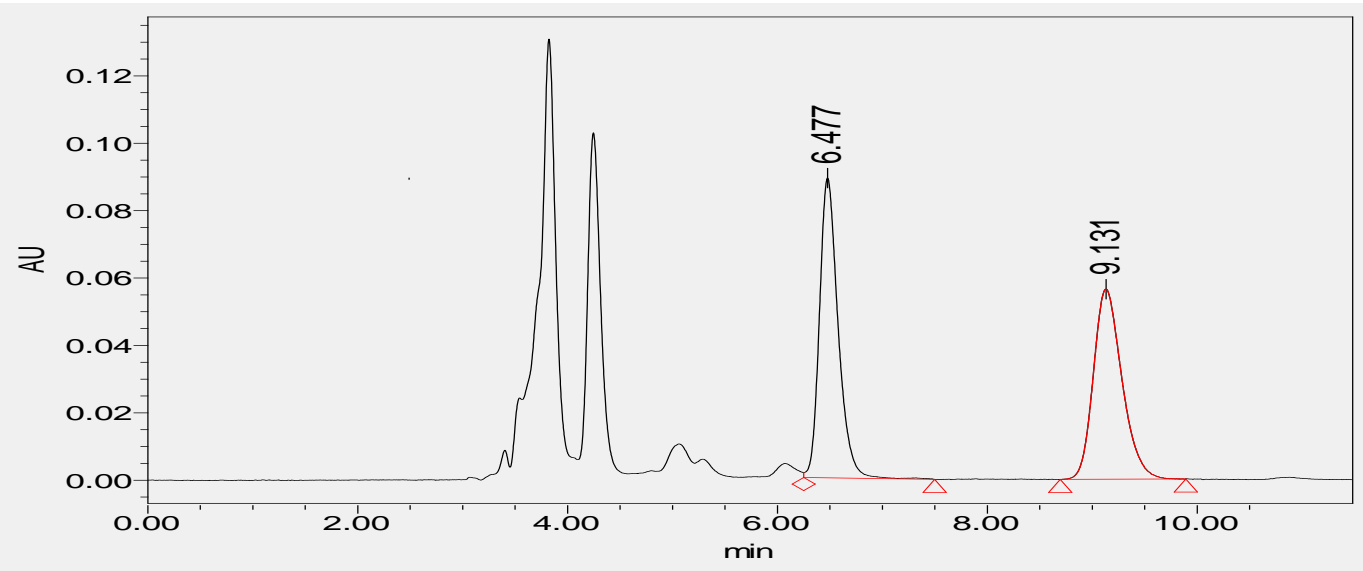

\begin{tabular}{|c|c|c|c|c|c|}
\hline & Retention time & Area & $\%$ Area & Height & Integral type \\
\hline 1 & 6.477 & 1101256 & 50.82 & 88996 & $\mathrm{vb}$ \\
\hline 2 & 9.131 & 1065755 & 49.18 & 56405 & $\mathrm{bb}$ \\
\hline
\end{tabular}

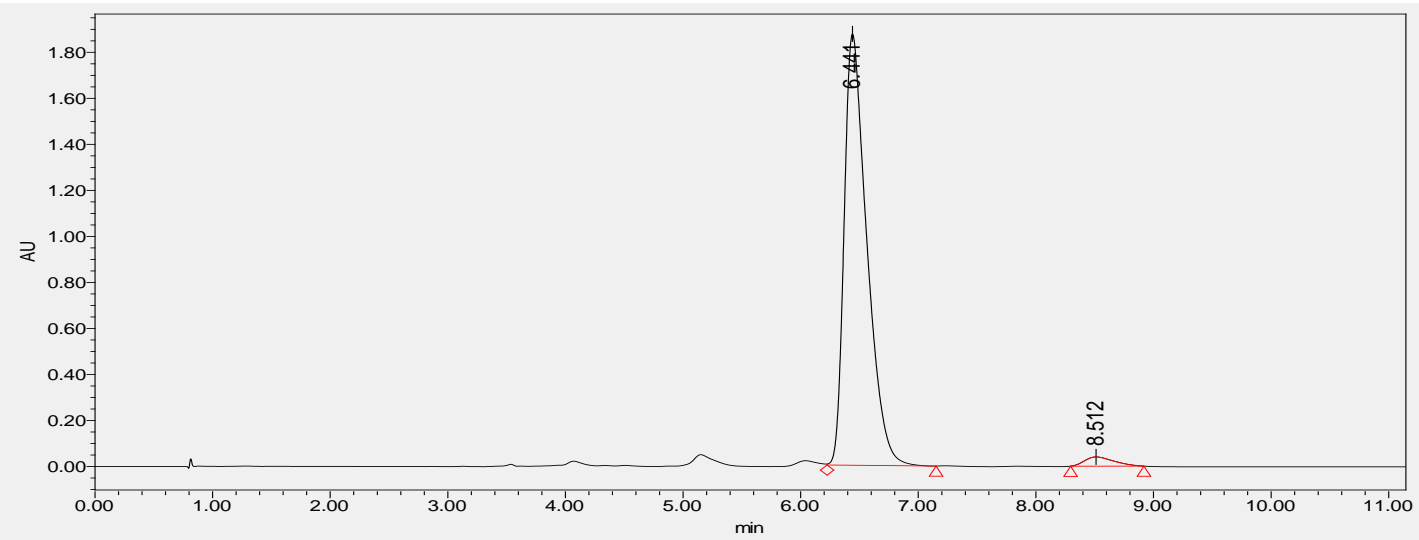

\begin{tabular}{|c|c|c|c|c|c|}
\hline & Retention time & Area & $\%$ Area & Height & Integral type \\
\hline 1 & 6.441 & 25072607 & 97.30 & 1874404 & $\mathrm{vb}$ \\
\hline 2 & 8.512 & 695034 & 2.70 & 40307 & $\mathrm{bb}$ \\
\hline
\end{tabular}


(R)-2-(1-methyl-2-oxo-3-phenyl-1,2-dihydronaphthalen-1-yl)cyclohexa-2,5-diene-1,4-dione

4j (Table 2)

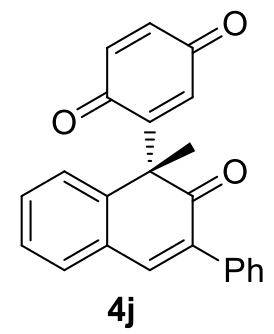

Chiralpak IC column, hexane/EtOH (9:1), flow rate $1.0 \mathrm{~mL} / \mathrm{min}$

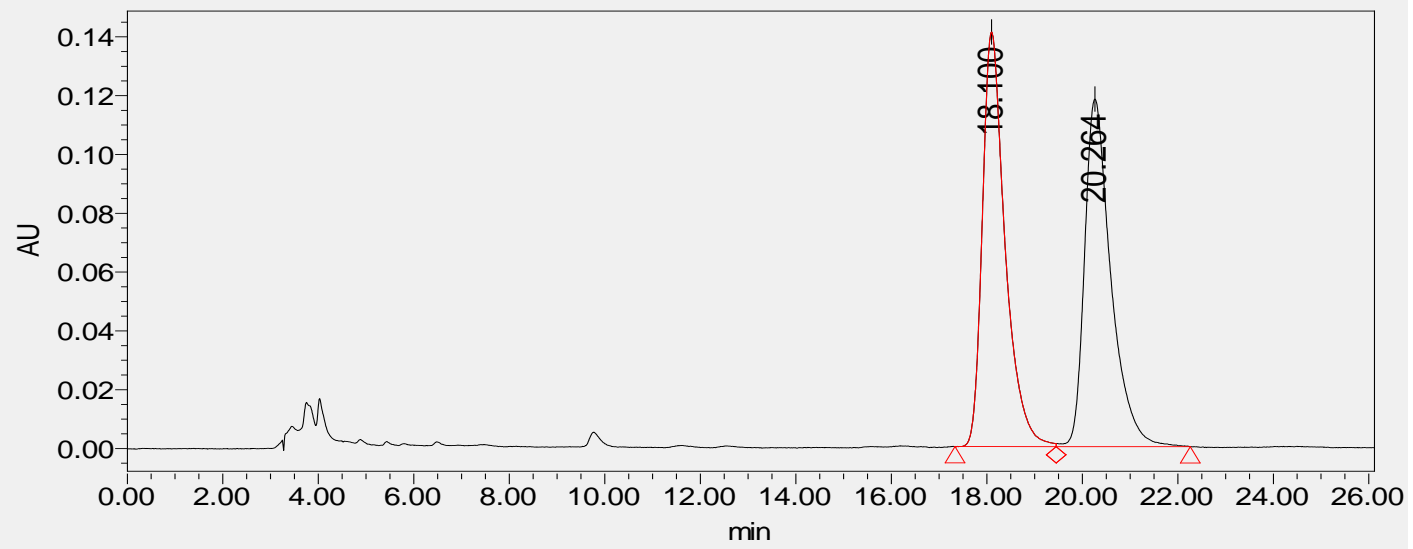

\begin{tabular}{|c|c|c|c|c|c|}
\hline & Retention time & Area & $\%$ Area & Height & Integral type \\
\hline 1 & 18.100 & 4665316 & 50.74 & 140942 & bv \\
\hline 2 & 20.264 & 4528427 & 49.26 & 118177 & $\mathrm{vb}$ \\
\hline
\end{tabular}

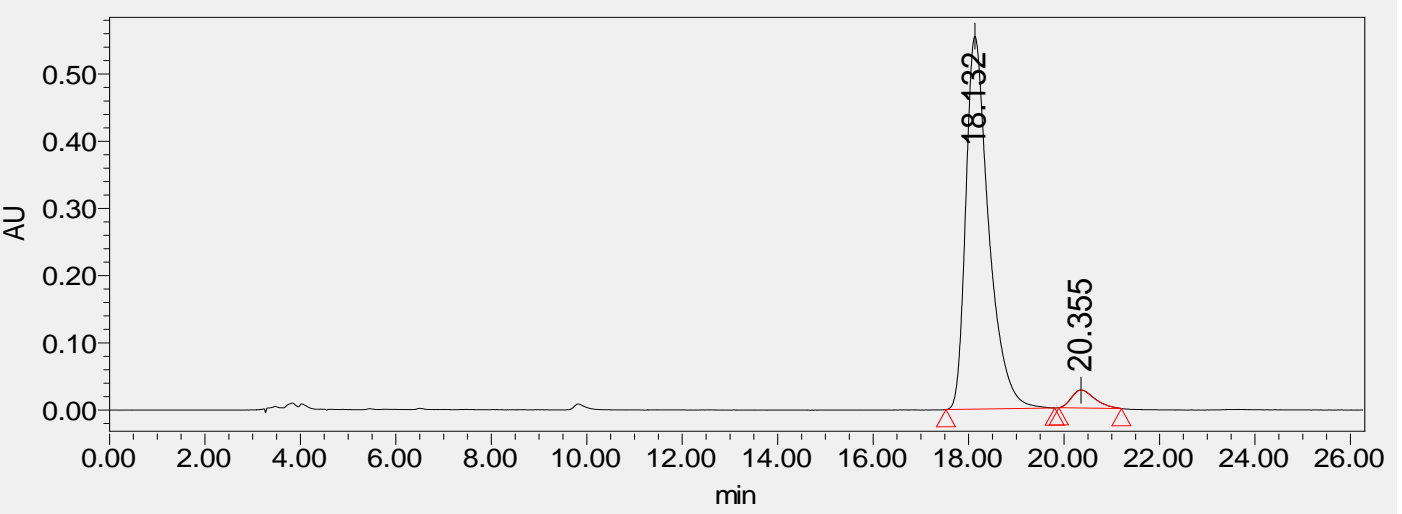

\begin{tabular}{|c|c|c|c|c|c|}
\hline & Retention time & Area & $\%$ Area & Height & Integral type \\
\hline 1 & 18.132 & 18511410 & 95.35 & 554939 & $\mathrm{bb}$ \\
\hline 2 & 20.355 & 902732 & 4.65 & 26745 & $\mathrm{bb}$ \\
\hline
\end{tabular}


(R)-2-(1-methyl-2-oxo-1,2-dihydronaphthalen-1-yl)cyclohexa-2,5-diene-1,4-dione

4k (Table 2)

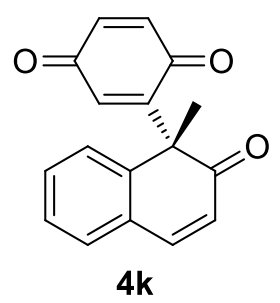

Chiralpak IA column, hexane/iPrOH (8:2), flow rate $1.0 \mathrm{~mL} / \mathrm{min}$

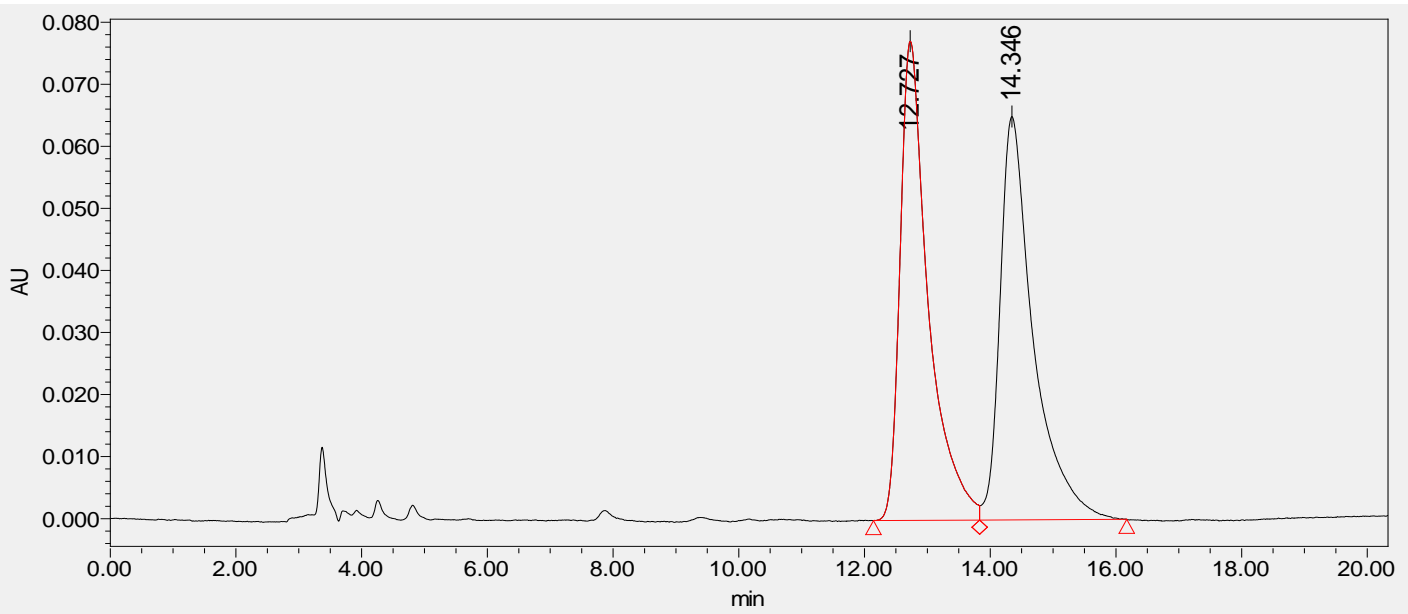

\begin{tabular}{|c|c|c|c|c|c|}
\hline & Retention time & Area & \% Area & Height & Integral type \\
\hline 1 & 12.727 & 2415085 & 50.26 & 77207 & $\mathrm{bv}$ \\
\hline 2 & 14.346 & 2390033 & 49.74 & 65006 & $\mathrm{vb}$ \\
\hline
\end{tabular}

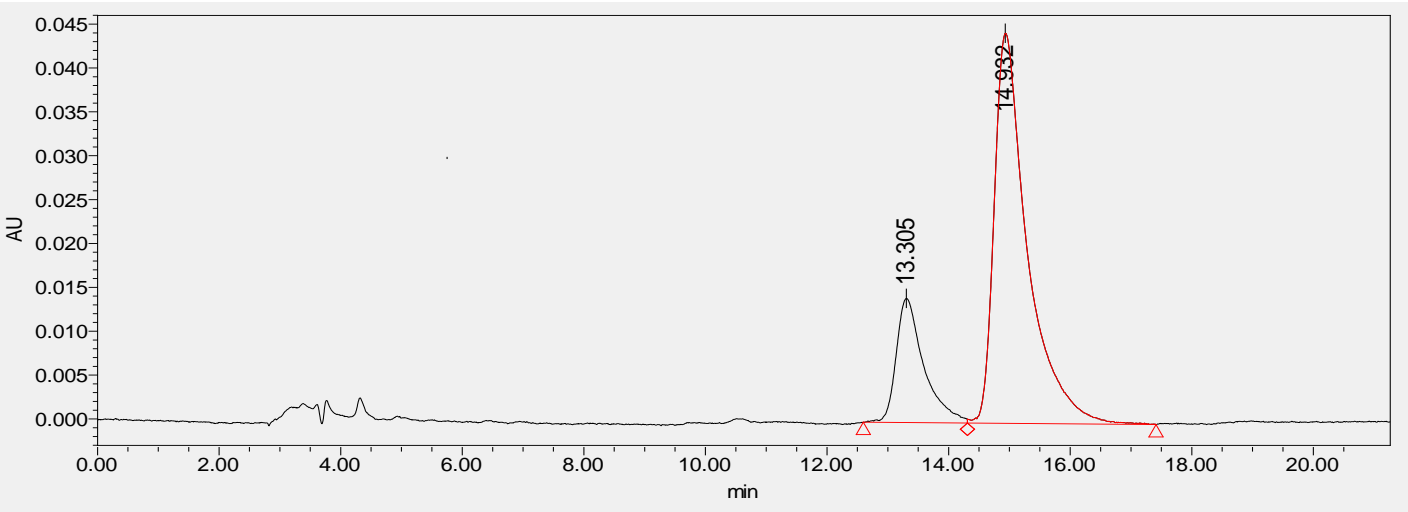

\begin{tabular}{|c|c|c|c|c|c|}
\hline & Retention time & Area & \% Area & Height & Integral type \\
\hline 1 & 13.305 & 445649 & 20.94 & 14156 & bv \\
\hline 2 & 14.932 & 1682452 & 79.06 & 44441 & $\mathrm{vb}$ \\
\hline
\end{tabular}


(R)-2-(1-ethyl-3-methyl-2-oxo-1,2-dihydronaphthalen-1-yl)cyclohexa-2,5-diene-1,4-dione

$4 \mathrm{~m}$ (Table 2)

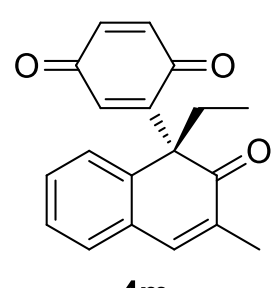

$4 \mathrm{~m}$

Chiralpak IC column, hexane/EtOH (9:1), flow rate $1.0 \mathrm{~mL} / \mathrm{min}$

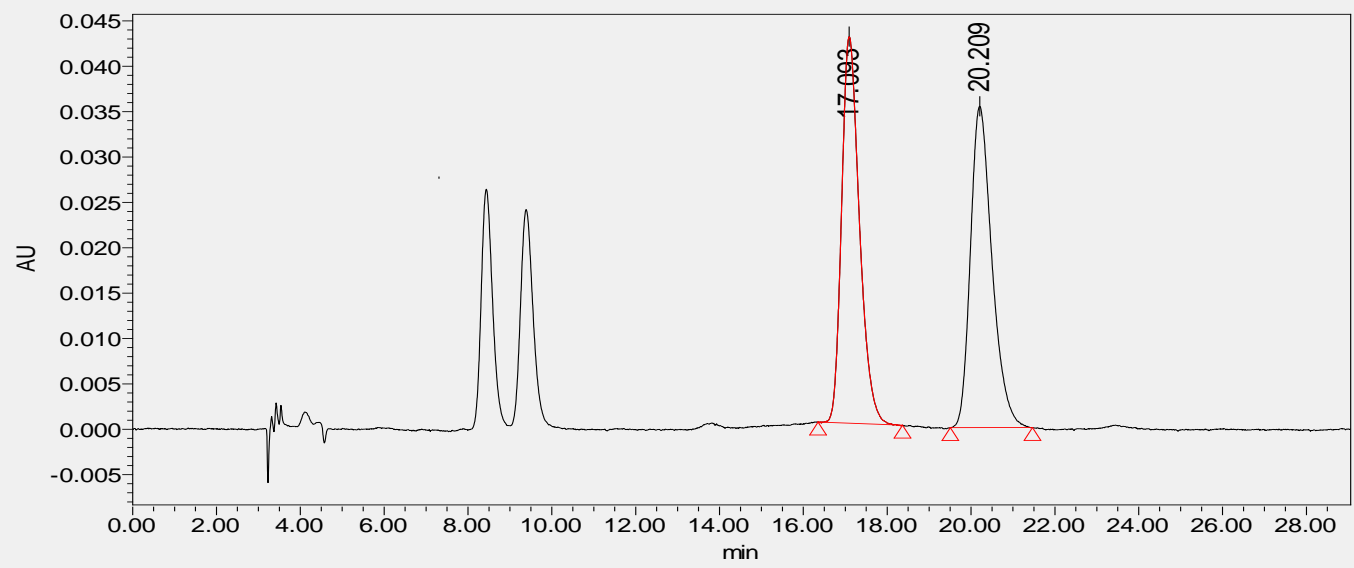

\begin{tabular}{|c|c|c|c|c|c|}
\hline & Retention time & Area & \% Area & Height & Integral type \\
\hline 1 & 17.093 & 1258384 & 49.89 & 42592 & bb \\
\hline 2 & 20.209 & 1263874 & 50.11 & 35417 & $\mathrm{bb}$ \\
\hline
\end{tabular}

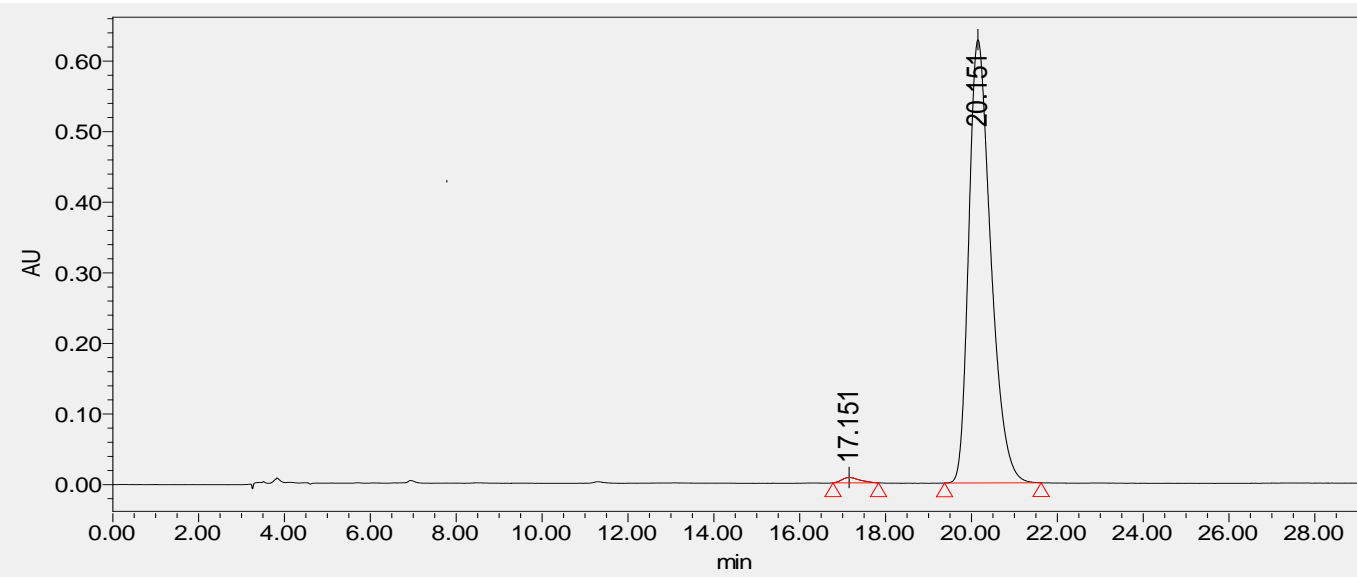

\begin{tabular}{|c|c|c|c|c|c|}
\hline & Retention time & Area & $\%$ Area & Height & Integral type \\
\hline 1 & 17.151 & 221407 & 0.98 & 7715 & $\mathrm{bb}$ \\
\hline 2 & 20.151 & 22316737 & 99.02 & 627929 & $\mathrm{bb}$ \\
\hline
\end{tabular}


(R)-2-(3-methyl-2-oxo-1-propyl-1,2-dihydronaphthalen-1-yl)cyclohexa-2,5-diene-1,4-dione

4n (Table 2)

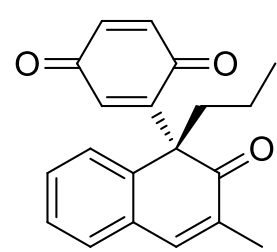

$4 n$

Chiralpak IC column, hexane/EtOH (8:2), flow rate $1.0 \mathrm{~mL} / \mathrm{min}$

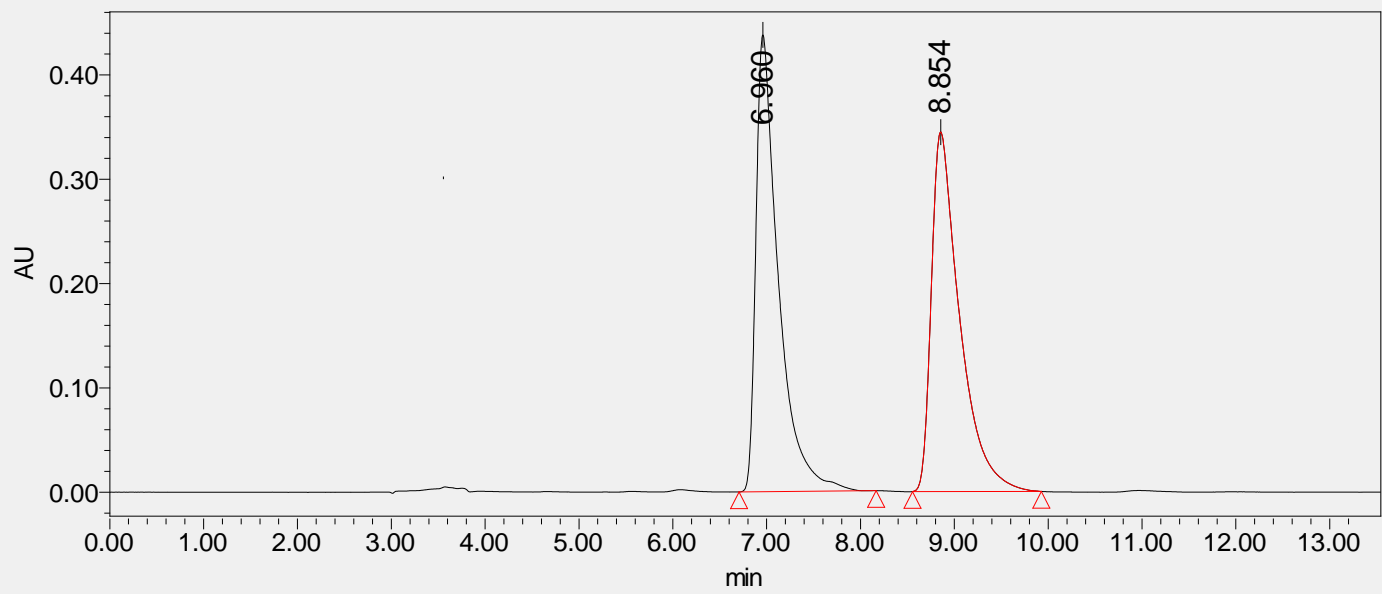

\begin{tabular}{|c|c|c|c|c|c|}
\hline & Retention time & Area & $\%$ Area & Height & Integral type \\
\hline 1 & 6.960 & 7280866 & 50.18 & 437792 & bb \\
\hline 2 & 8.854 & 7228408 & 49.82 & 344624 & bb \\
\hline
\end{tabular}

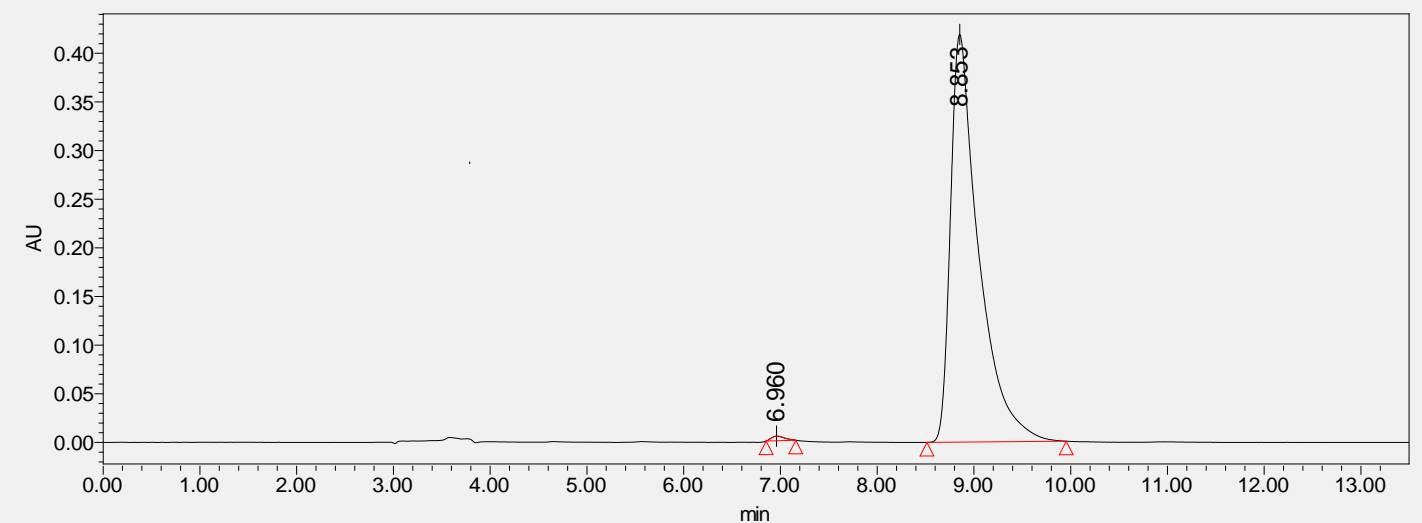

\begin{tabular}{|c|c|c|c|c|c|}
\hline & Retention time & Area & $\%$ Area & Height & Integral type \\
\hline 1 & 6.960 & 45632 & 0.54 & 4782 & $\mathrm{bb}$ \\
\hline 2 & 8.853 & 8471271 & 99.46 & 419168 & $\mathrm{bb}$ \\
\hline
\end{tabular}


(R)-2-(1-allyl-3-methyl-2-oxo-1,2-dihydronaphthalen-1-yl)cyclohexa-2,5-diene-1,4-dione

40 (Table 2)

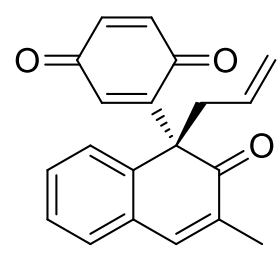

40

Chiralpak IC column, hexane/EtOH (9:1), flow rate $1.0 \mathrm{~mL} / \mathrm{min}$

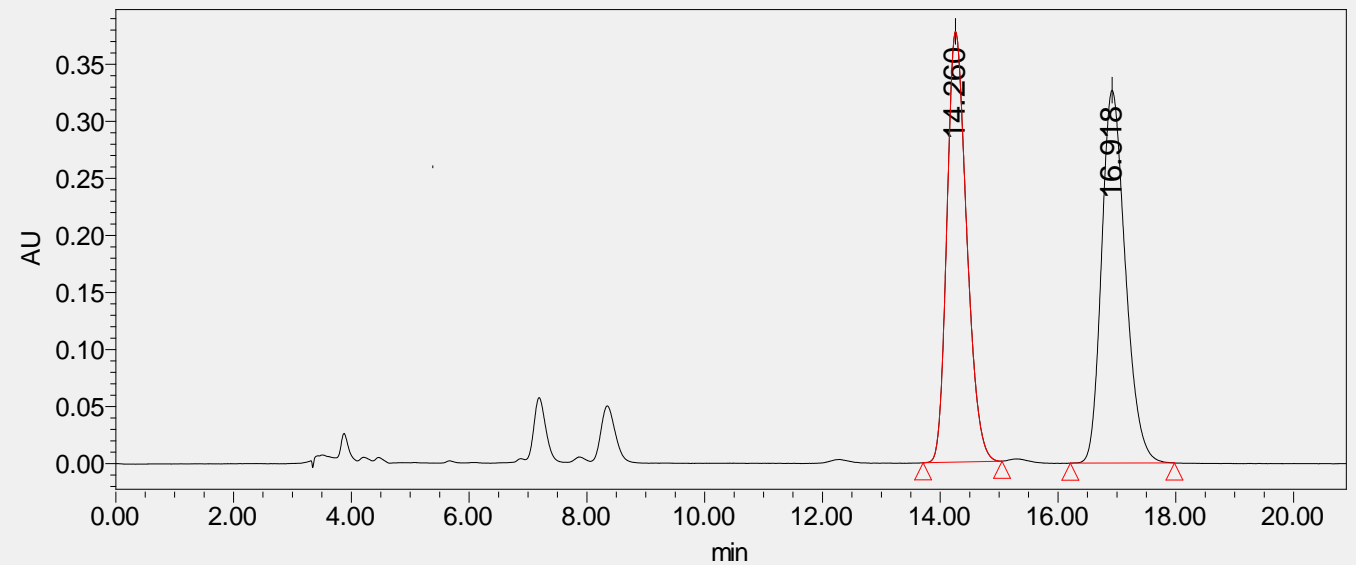

\begin{tabular}{|c|c|c|c|c|c|}
\hline & Retention time & Area & $\%$ Area & Height & Integral type \\
\hline 1 & 14.260 & 8819131 & 49.06 & 377465 & $\mathrm{bb}$ \\
\hline 2 & 16.918 & 9158585 & 50.94 & 326711 & $\mathrm{bb}$ \\
\hline
\end{tabular}

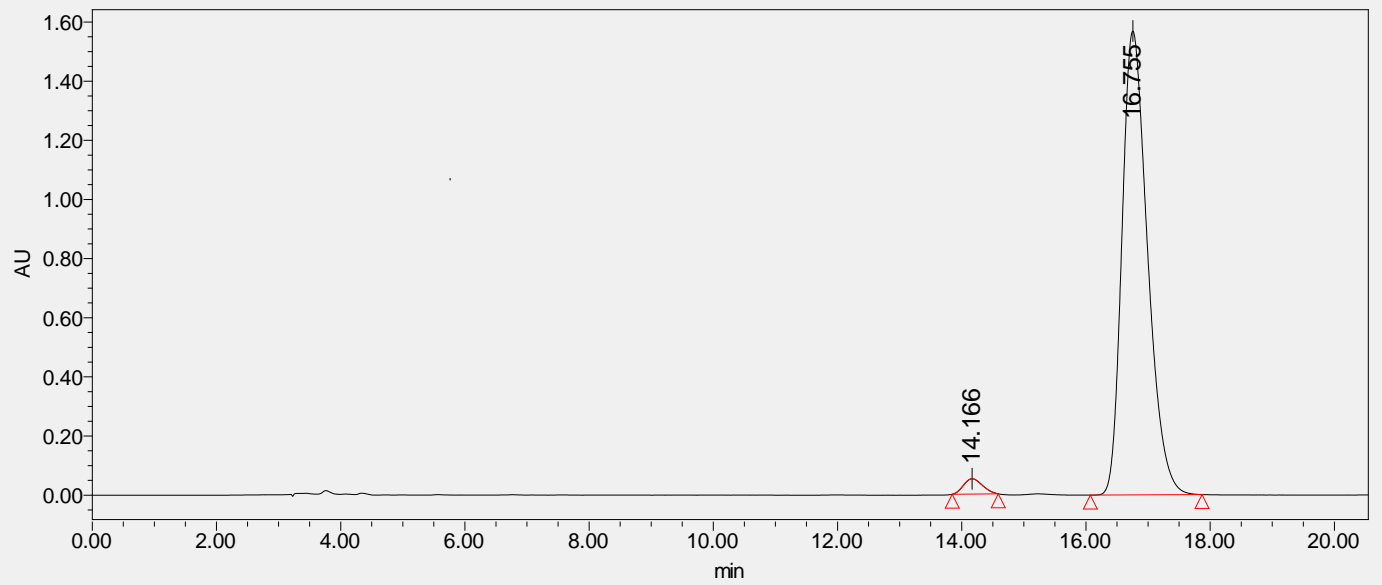

\begin{tabular}{|c|c|c|c|c|c|}
\hline & Retention time & Area & $\%$ Area & Height & Integral type \\
\hline 1 & 14.166 & 1098694 & 2.40 & 52052 & $\mathrm{bb}$ \\
\hline 2 & 16.755 & 44730385 & 97.60 & 1568599 & $\mathrm{bb}$ \\
\hline
\end{tabular}


$4 p$ (Scheme 3)

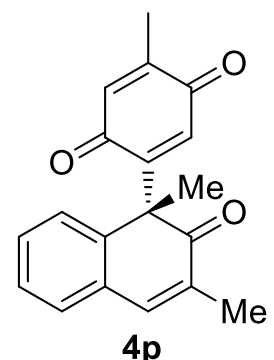

Chiralpak IC column, hexane/EtOH (8:2), flow rate $1.0 \mathrm{~mL} / \mathrm{min}$

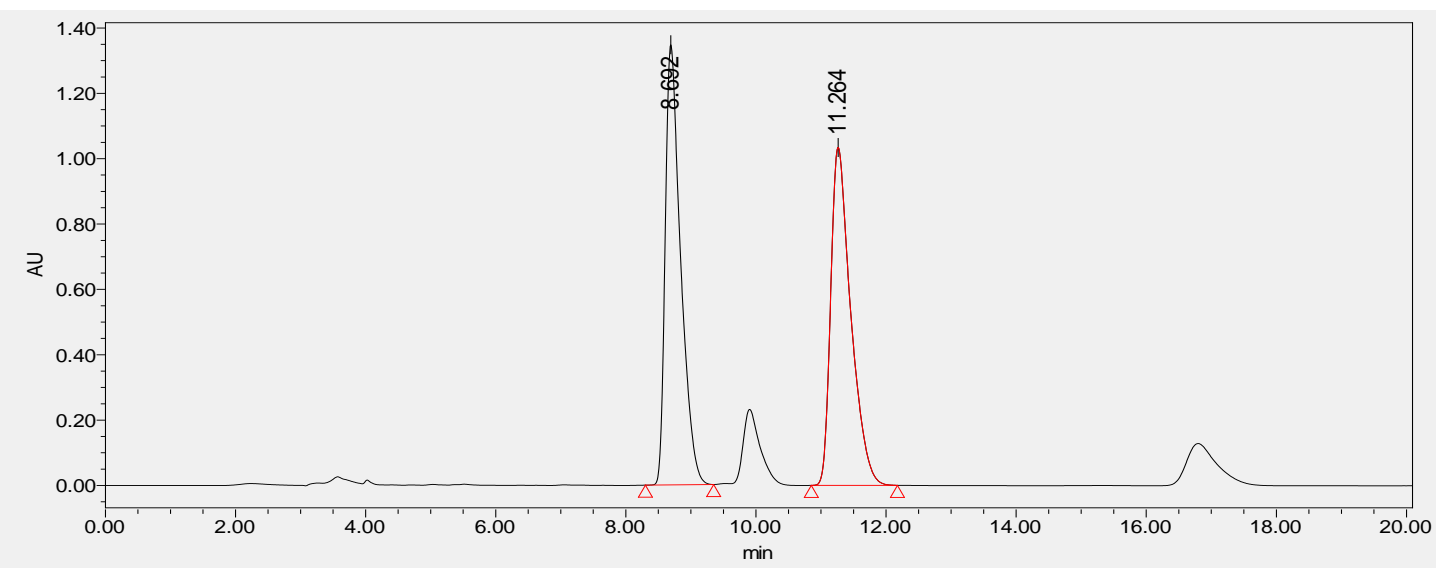

\begin{tabular}{|c|c|c|c|c|c|}
\hline & Retention time & Area & \% Area & Height & Integral type \\
\hline 1 & 8.692 & 22133515 & 49.95 & 1346651 & bb \\
\hline 2 & 11.264 & 22177012 & 50.05 & 1034359 & $\mathrm{bb}$ \\
\hline
\end{tabular}

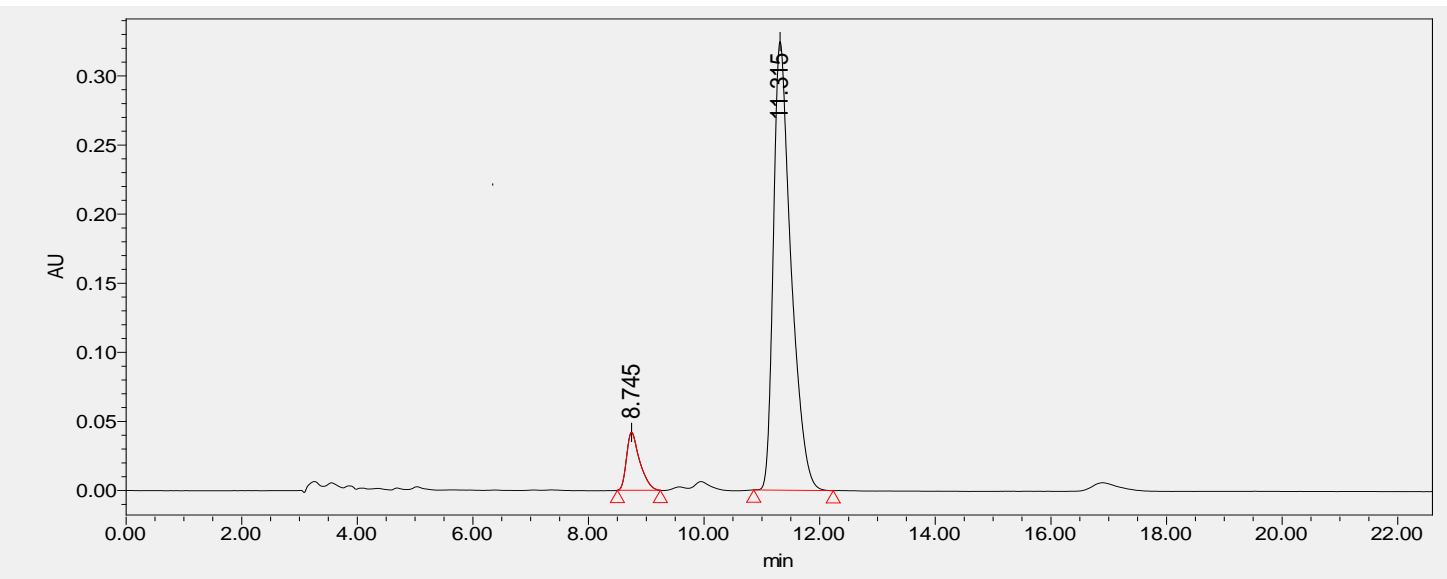

\begin{tabular}{|c|c|c|c|c|c|}
\hline & Retention time & Area & \% Area & Height & Integral type \\
\hline 1 & 8.745 & 663970 & 8.82 & 42031 & $\mathrm{bb}$ \\
\hline 2 & 11.315 & 6864662 & 91.18 & 324603 & $\mathrm{bb}$ \\
\hline
\end{tabular}


4q (Scheme 3)

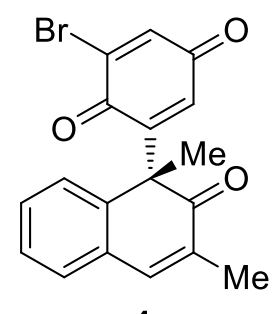

$4 \mathbf{q}$

Chiralpak IA column, hexane/EtOH (8:2), flow rate $1.0 \mathrm{~mL} / \mathrm{min}$

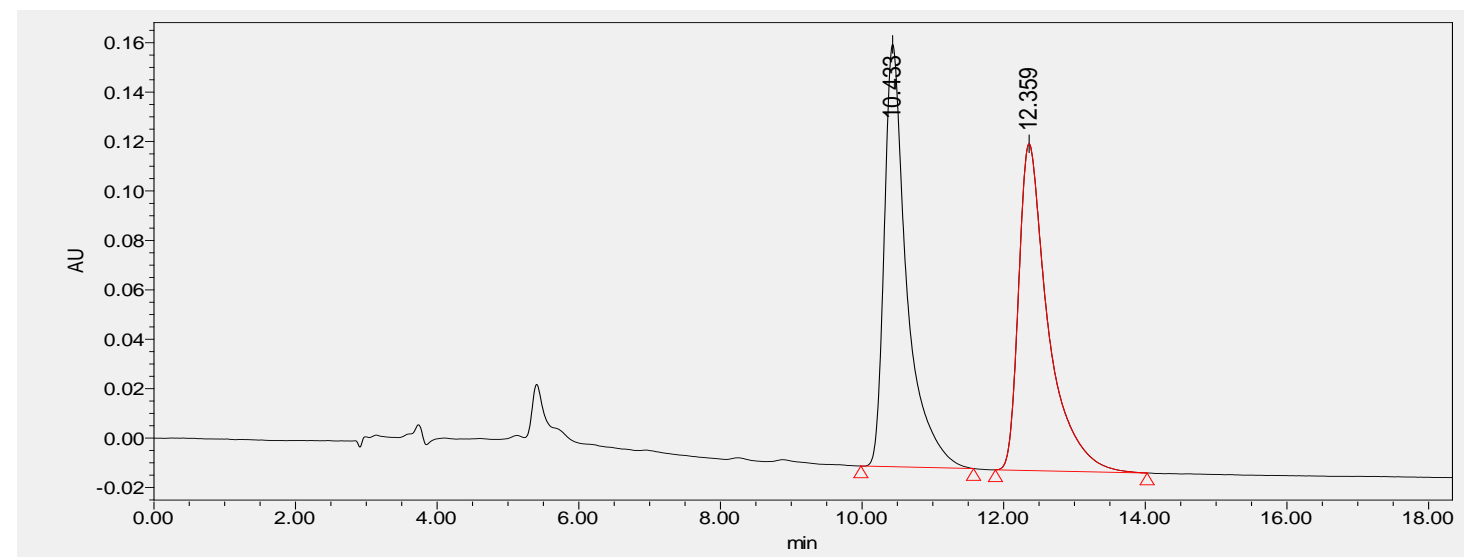

\begin{tabular}{|c|c|c|c|c|c|}
\hline & Retention time & Area & $\%$ Area & Height & Integral type \\
\hline 1 & 10.433 & 3768801 & 49.70 & 170907 & $\mathrm{bb}$ \\
\hline 2 & 12.359 & 3814506 & 50.30 & 132234 & $\mathrm{bb}$ \\
\hline
\end{tabular}

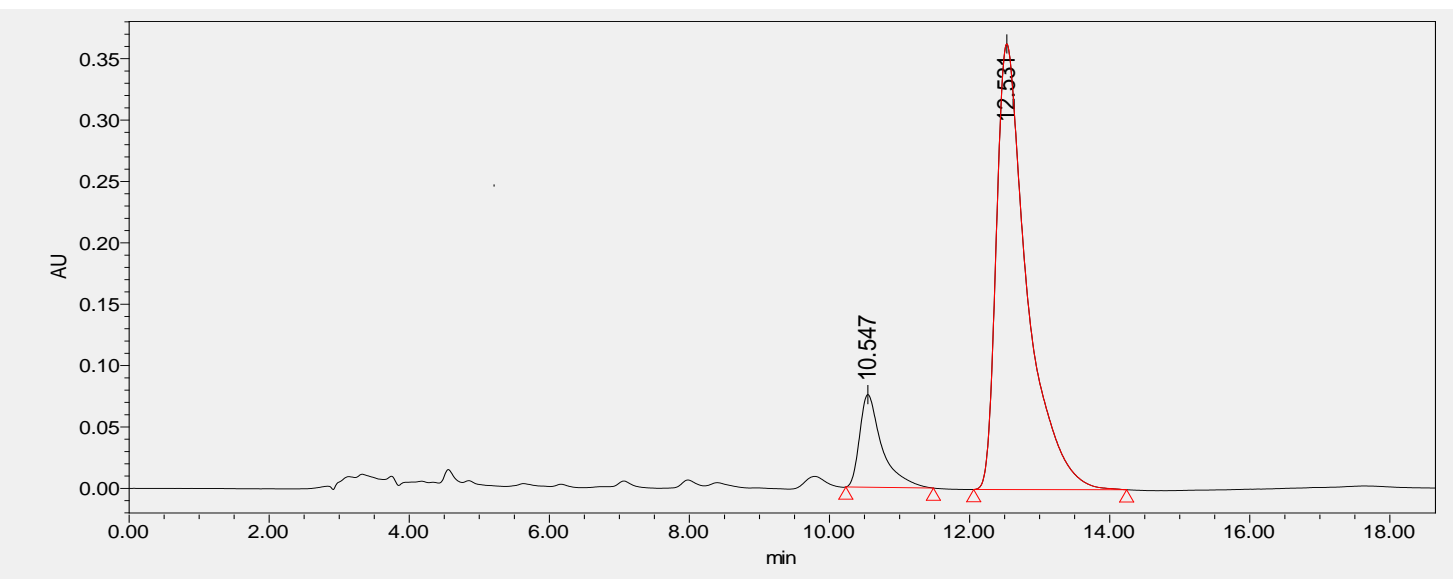

\begin{tabular}{|c|c|c|c|c|c|}
\hline & Retention time & Area & $\%$ Area & Height & Integral type \\
\hline 1 & 10.547 & 1670398 & 13.11 & 75528 & $\mathrm{bb}$ \\
\hline 2 & 12.531 & 11068416 & 86.89 & 363065 & $\mathrm{bb}$ \\
\hline
\end{tabular}


(S)-2-bromo-6-(1,3-dimethyl-2-oxo-1,2-dihydronaphthalen-1-yl)cyclohexa-2,5-diene-1,4-dione

4q' (Scheme 3)

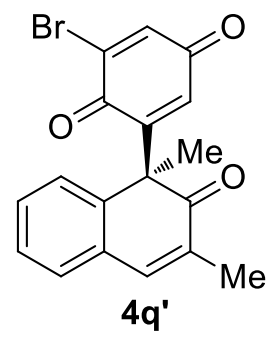

Chiralpak IA column, hexane/EtOH (8:2), flow rate $1.0 \mathrm{~mL} / \mathrm{min}$

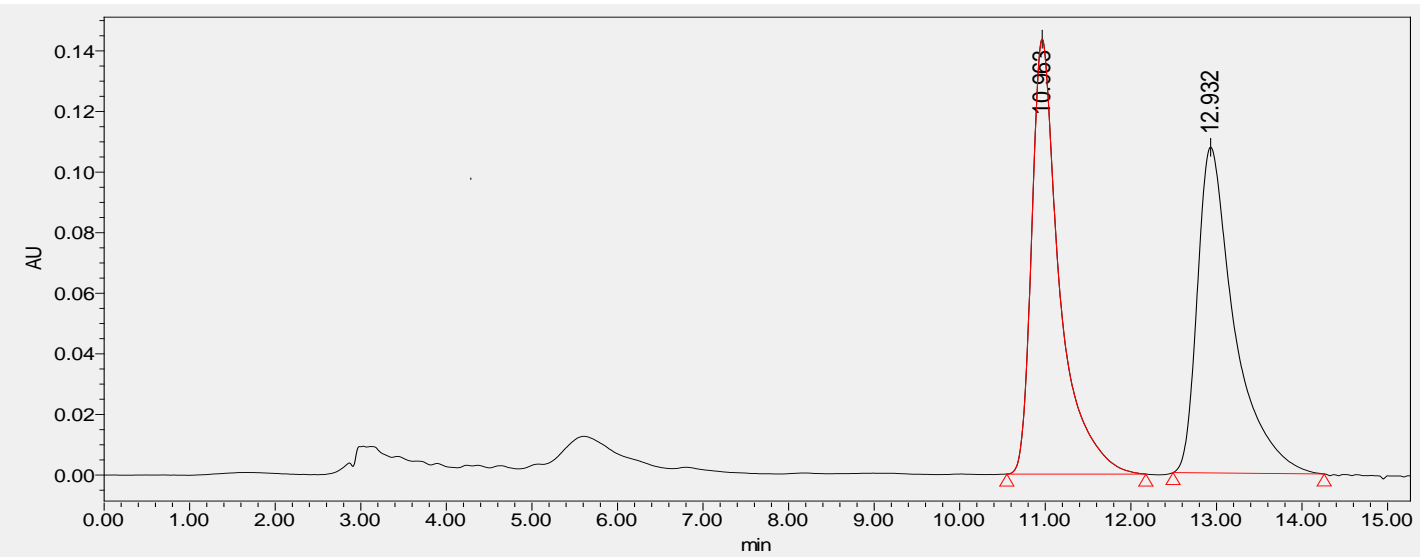

\begin{tabular}{|l|c|c|c|c|c|}
\hline & Retention time & Area & \% Area & Height & Integral type \\
\hline 1 & 10.963 & 3257438 & 50.31 & 143605 & $\mathrm{bb}$ \\
\hline 2 & 12.932 & 3216723 & 49.69 & 107532 & $\mathrm{bb}$ \\
\hline
\end{tabular}

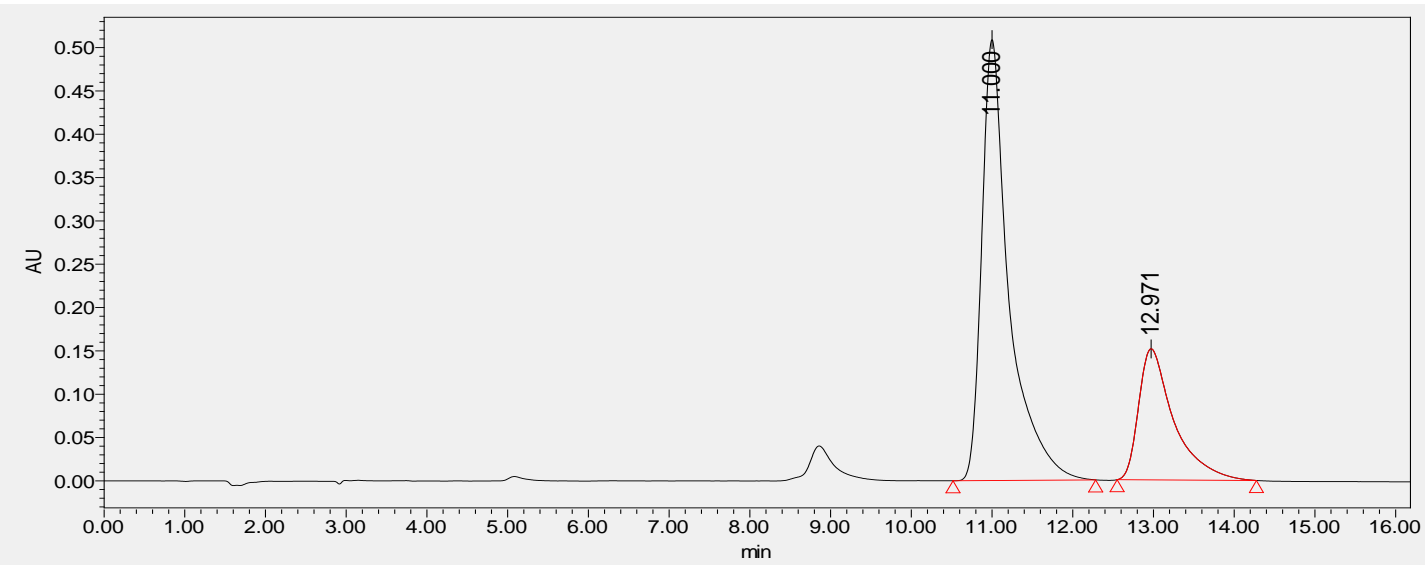

\begin{tabular}{|c|c|c|c|c|c|}
\hline & Retention time & Area & $\%$ Area & Height & Integral type \\
\hline 1 & 11.000 & 11921945 & 72.26 & 508799 & $\mathrm{bb}$ \\
\hline 2 & 12.971 & 4576798 & 27.74 & 151276 & $\mathrm{bb}$ \\
\hline
\end{tabular}


4r (Scheme 3)

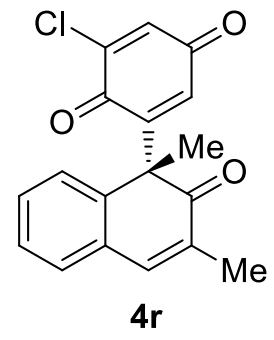

Chiralpak IC column, hexane/EtOH (9:1), flow rate $1.0 \mathrm{~mL} / \mathrm{min}$

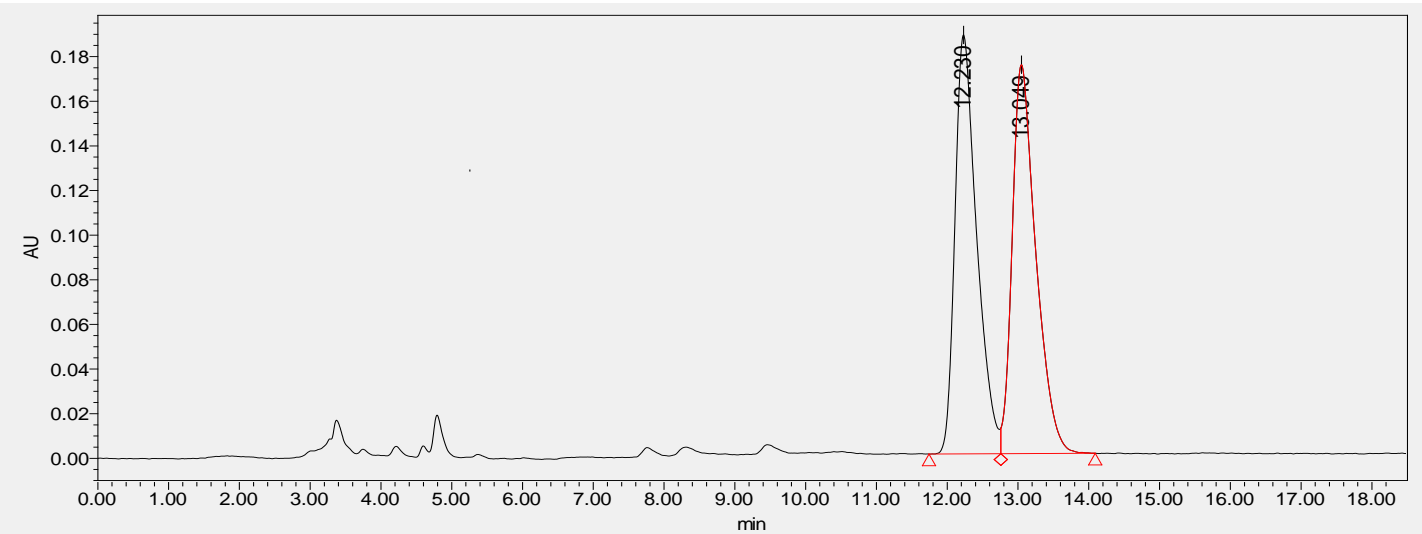

\begin{tabular}{|l|c|c|c|c|c|}
\hline & Retention time & Area & \% Area & Height & Integral type \\
\hline 1 & 12.230 & 4018701 & 49.83 & 187742 & bv \\
\hline 2 & 13.049 & 4046684 & 50.17 & 174244 & $\mathrm{vb}$ \\
\hline
\end{tabular}

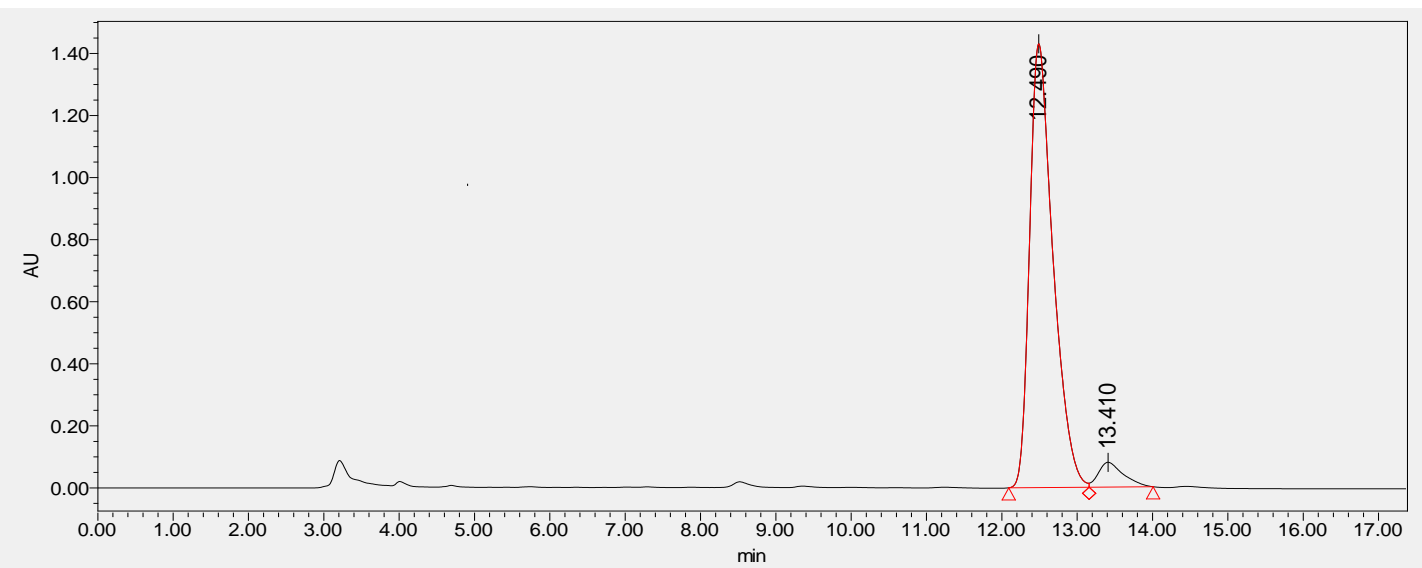

\begin{tabular}{|l|c|c|c|c|c|}
\hline & Retention time & Area & $\%$ Area & Height & Integral type \\
\hline 1 & 12.490 & 30983103 & 94.39 & 1430641 & bv \\
\hline 2 & 13.410 & 1842602 & 5.61 & 80003 & vb \\
\hline
\end{tabular}




\section{S (Scheme 3)}<smiles>CC1=Cc2ccccc2[C@@](C)(C2=CC(=O)C(Cl)=CC2=O)C1=O</smiles>

Chiralpak IA column, hexane/EtOH (8:2), flow rate $1.0 \mathrm{~mL} / \mathrm{min}$

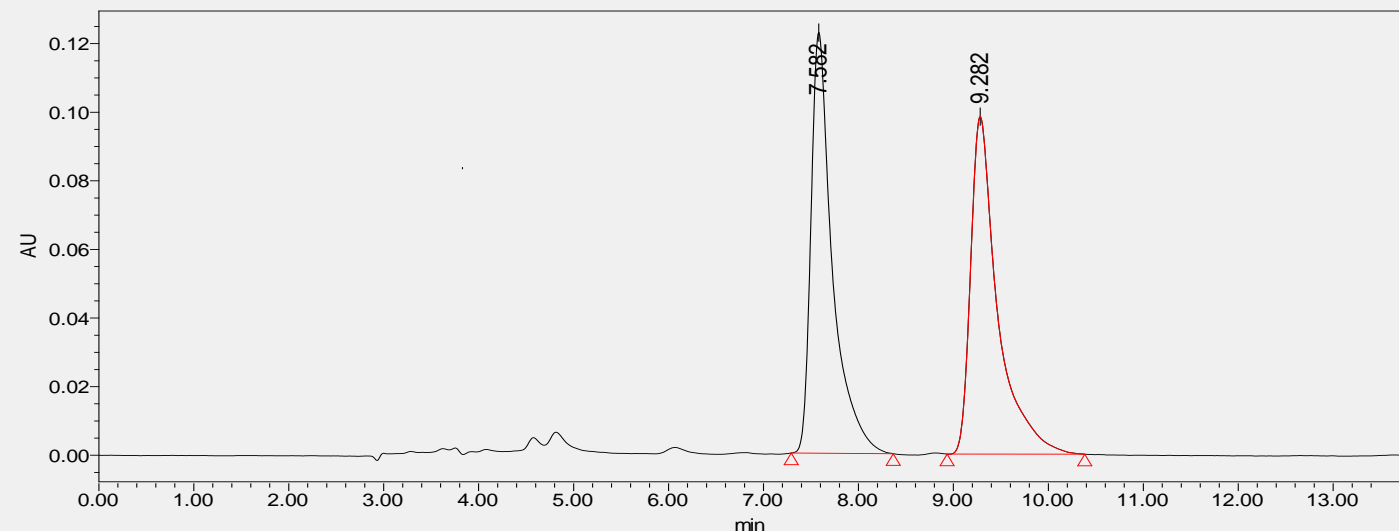

\begin{tabular}{|c|c|c|c|c|c|}
\hline & Retention time & Area & $\%$ Area & Height & Integral type \\
\hline 1 & 7.582 & 1927828 & 49.83 & 122659 & $\mathrm{bb}$ \\
\hline 2 & 9.282 & 1941197 & 50.17 & 98423 & $\mathrm{bb}$ \\
\hline
\end{tabular}

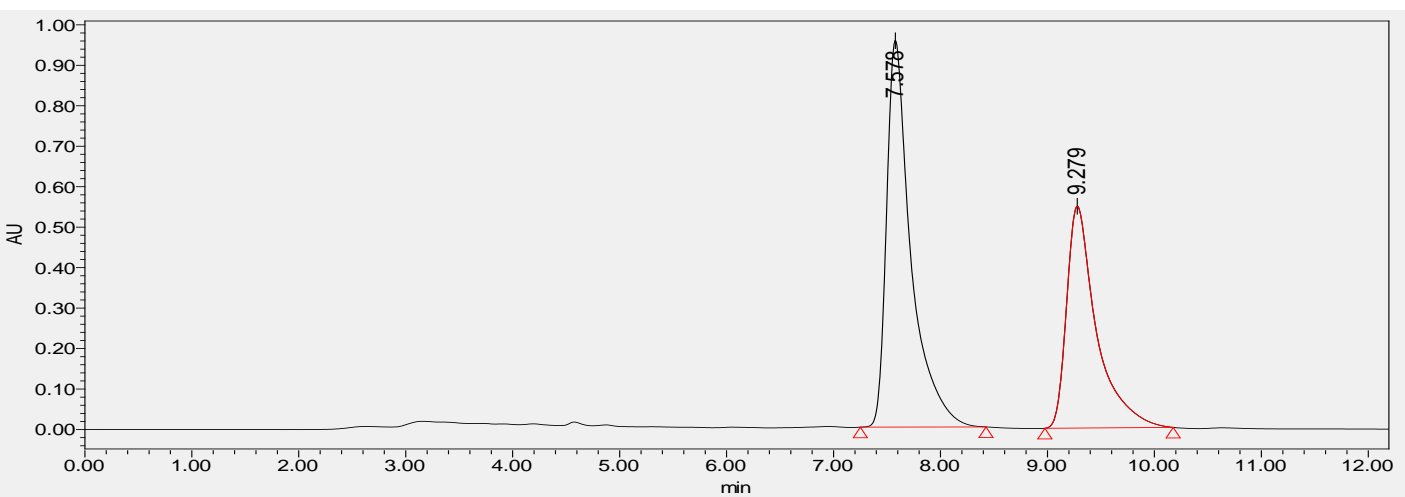

\begin{tabular}{|c|c|c|c|c|c|}
\hline & Retention time & Area & \% Area & Height & Integral type \\
\hline 1 & 7.578 & 15126910 & 58.81 & 955940 & $\mathrm{bb}$ \\
\hline 2 & 9.279 & 10593369 & 41.19 & 548548 & $\mathrm{bb}$ \\
\hline
\end{tabular}


(6aR,11bR)-6,11b-dimethyl-6a,11b-dihydronaphtho[2,1-b]benzofuran-10-ol

5 (Scheme 3)

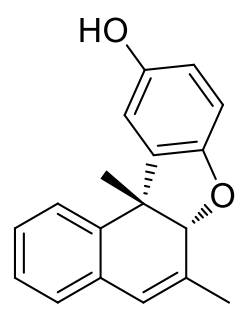

5

Chiralpak IA column, hexane/EtOH (95:5), flow rate $1.0 \mathrm{~mL} / \mathrm{min}$

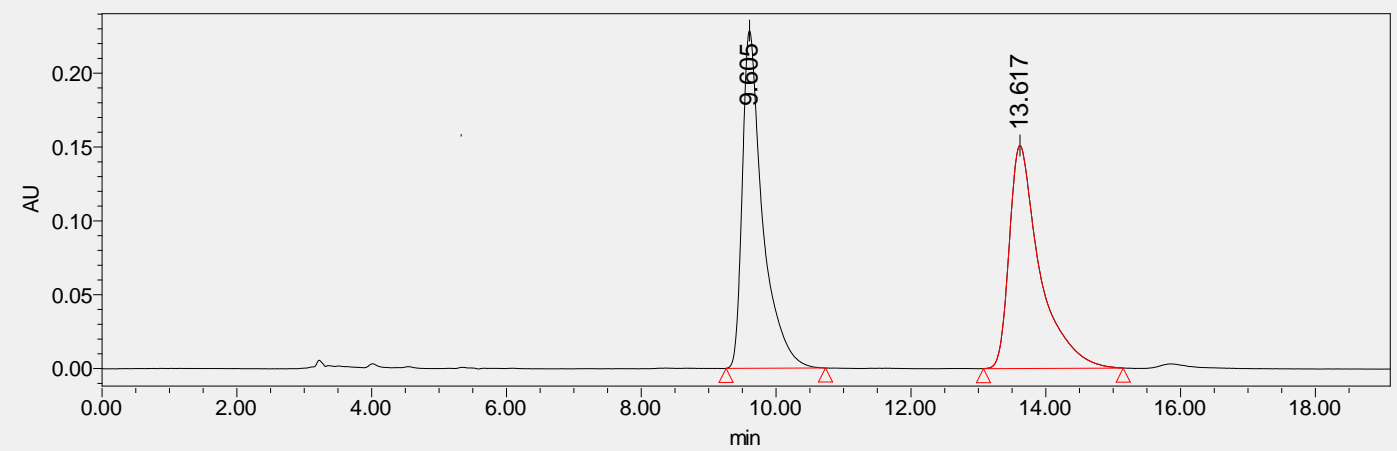

\begin{tabular}{|c|c|c|c|c|c|}
\hline & Retention time & Area & $\%$ Area & Height & Integral type \\
\hline 1 & 9.605 & 4755993 & 50.06 & 228538 & $\mathrm{bb}$ \\
\hline 2 & 13.617 & 4745510 & 49.94 & 150987 & $\mathrm{bb}$ \\
\hline
\end{tabular}

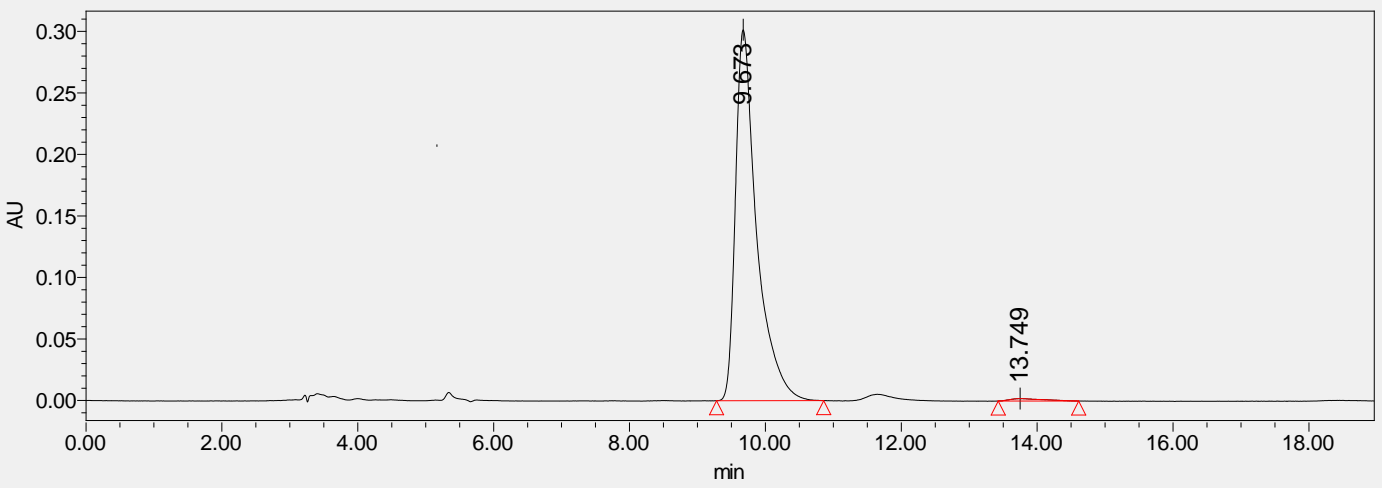

\begin{tabular}{|c|c|c|c|c|c|}
\hline & Retention time & Area & $\%$ Area & Height & Integral type \\
\hline 1 & 9.673 & 6525752 & 99.00 & 301449 & $\mathrm{bb}$ \\
\hline 2 & 13.749 & 65833 & 1.00 & 2018 & $\mathrm{bb}$ \\
\hline
\end{tabular}



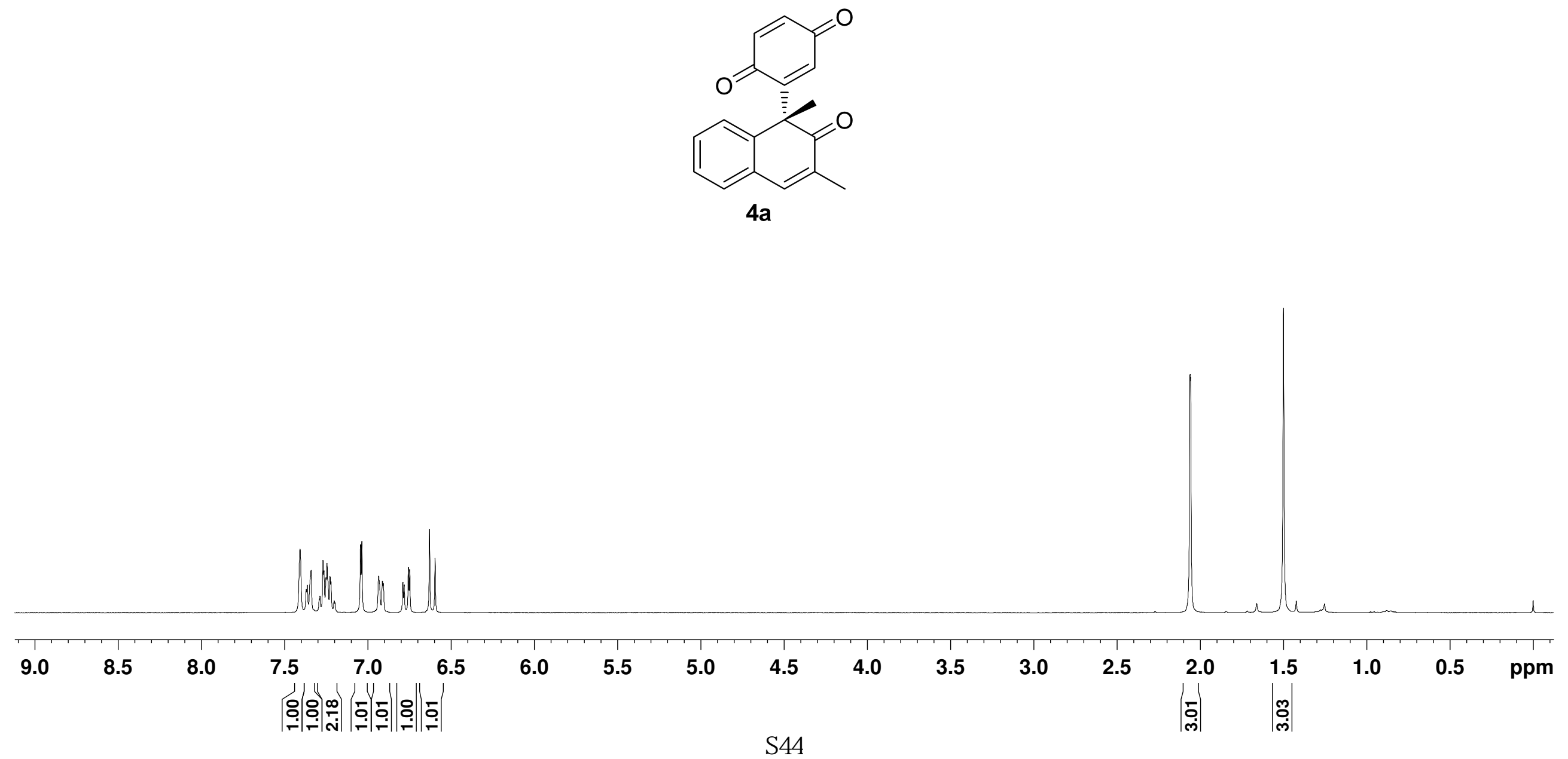


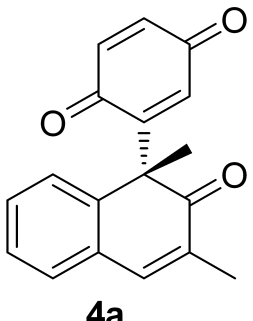

$4 a$
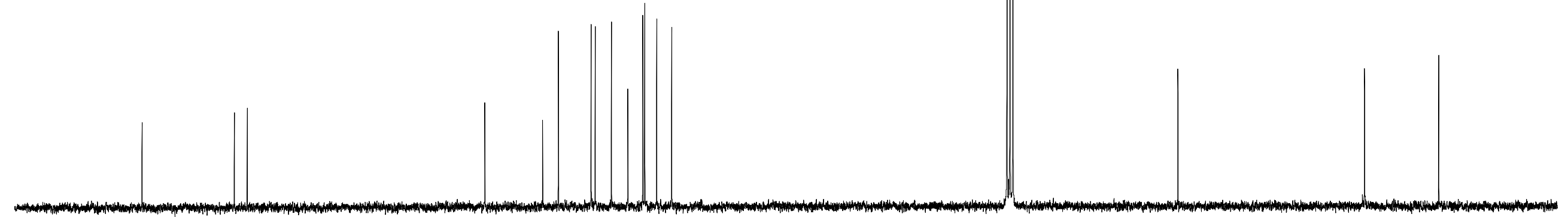


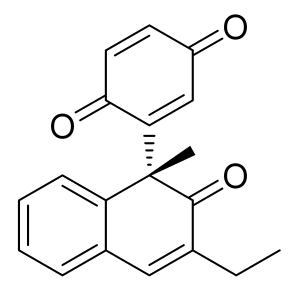

4b

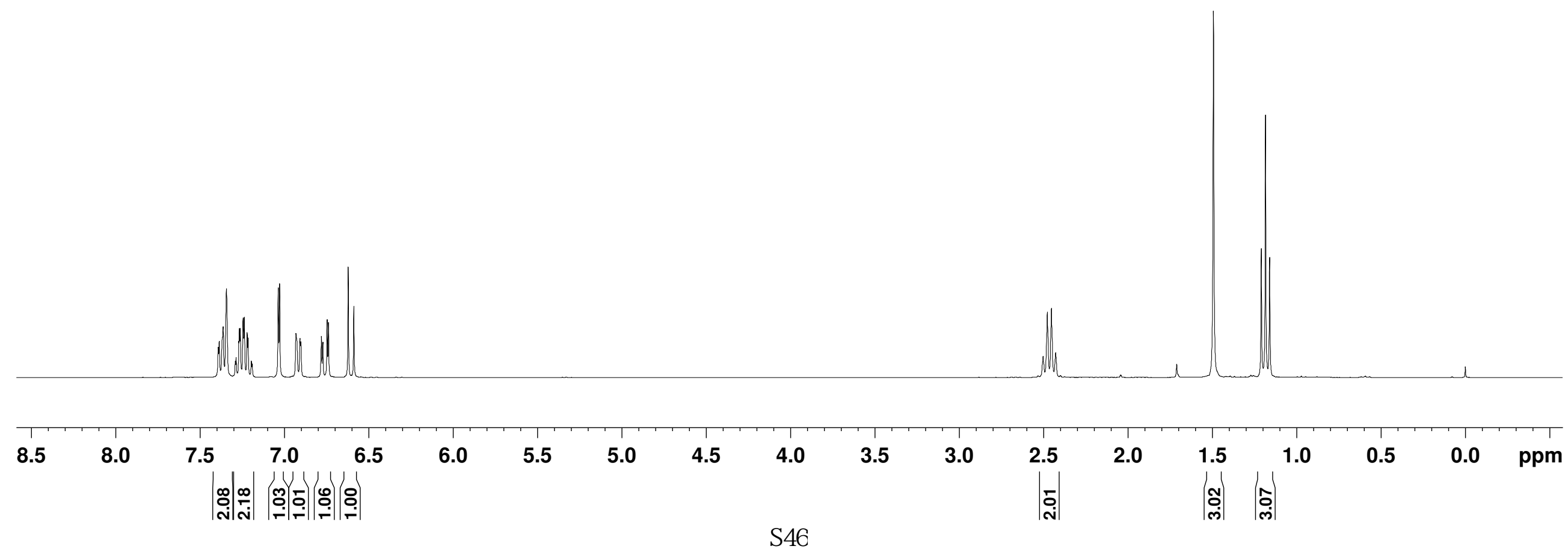



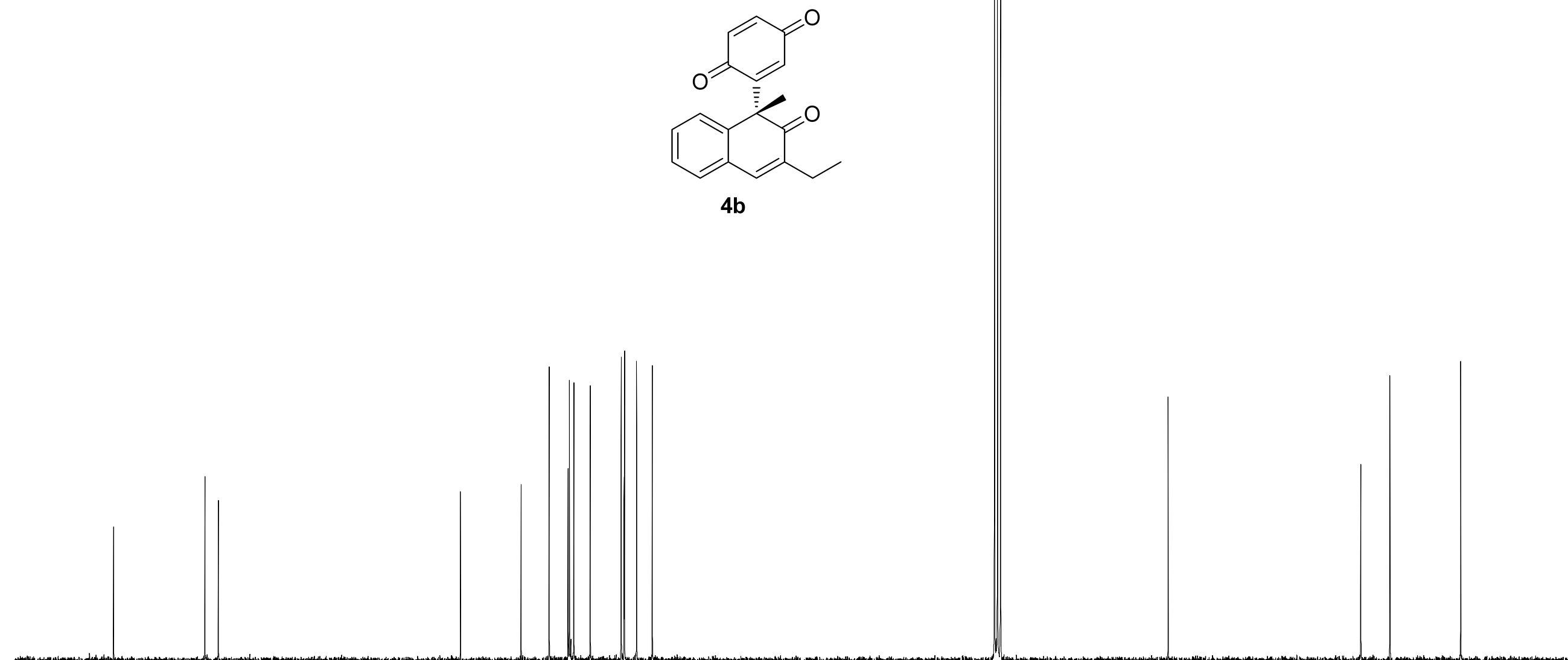


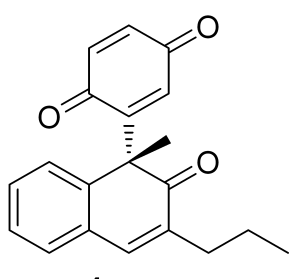

4c
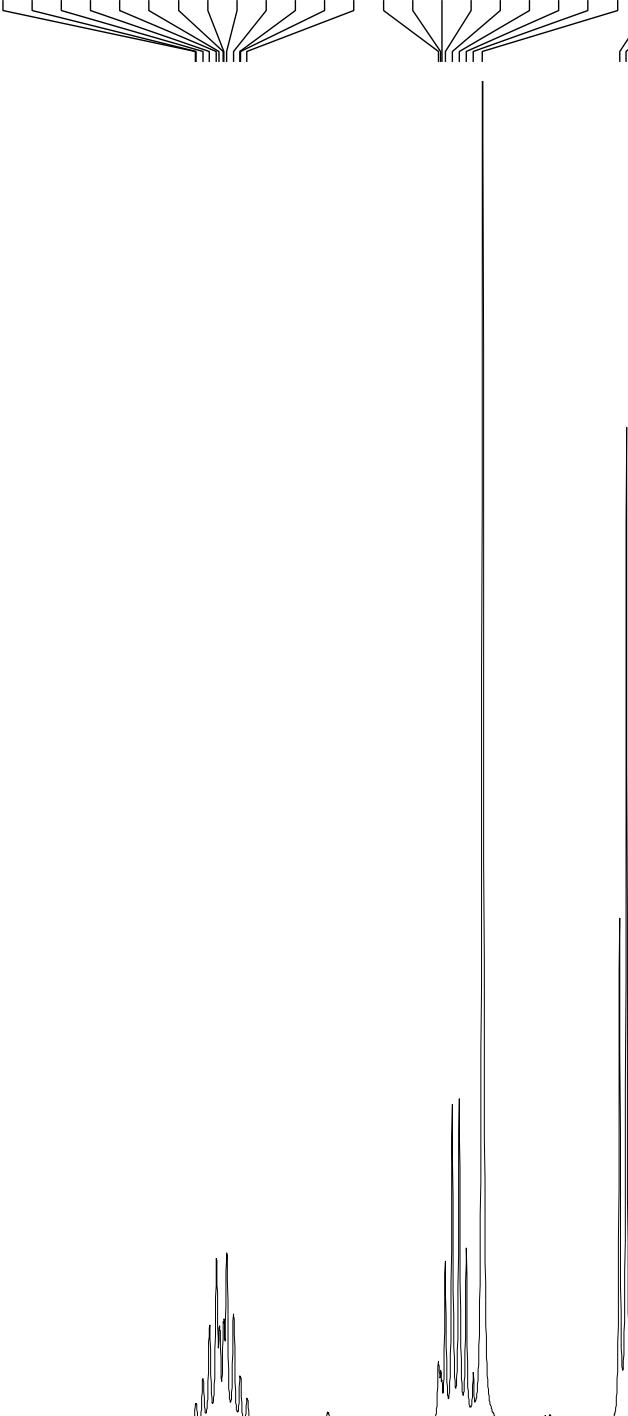

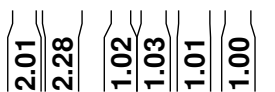

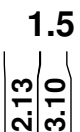

1.0

t:

ppm 

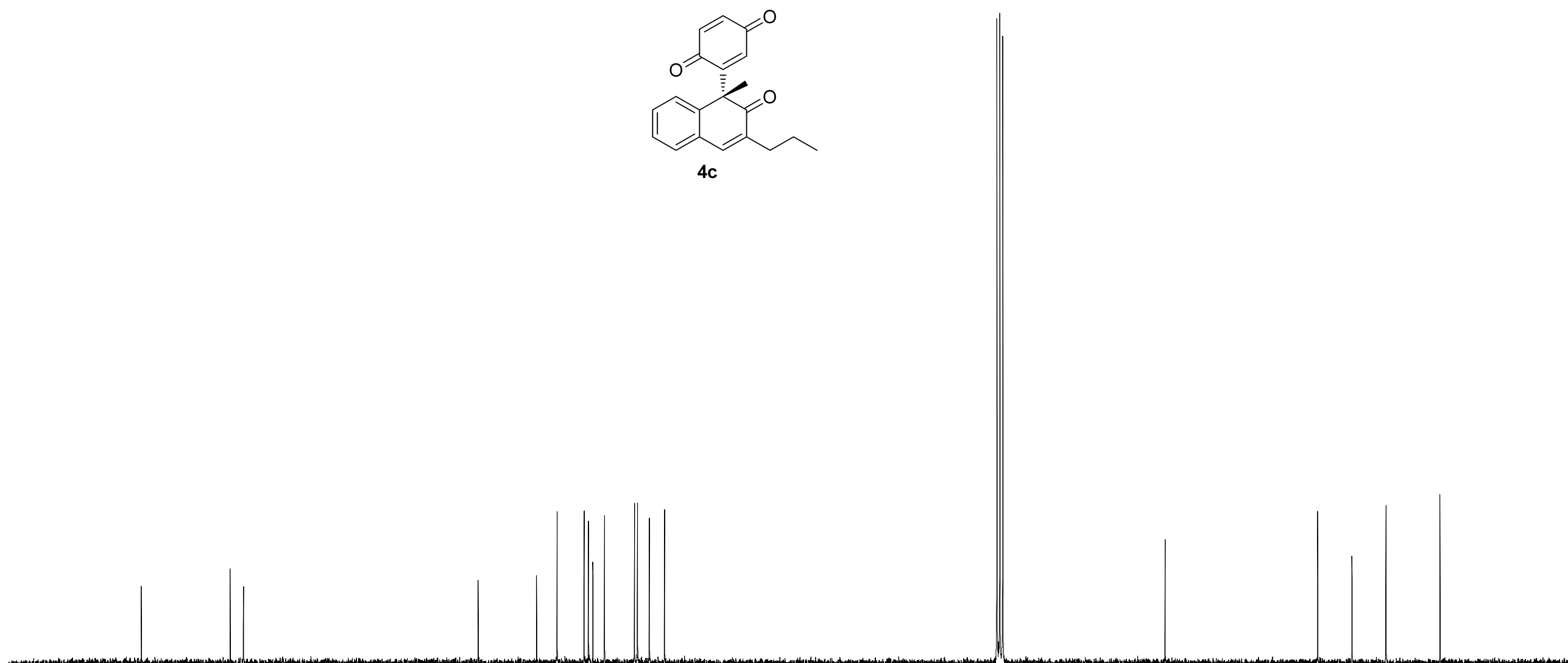


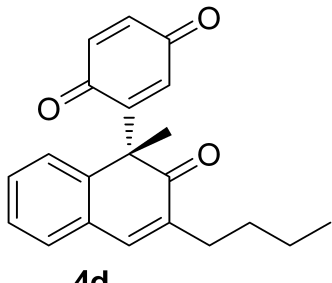

4d

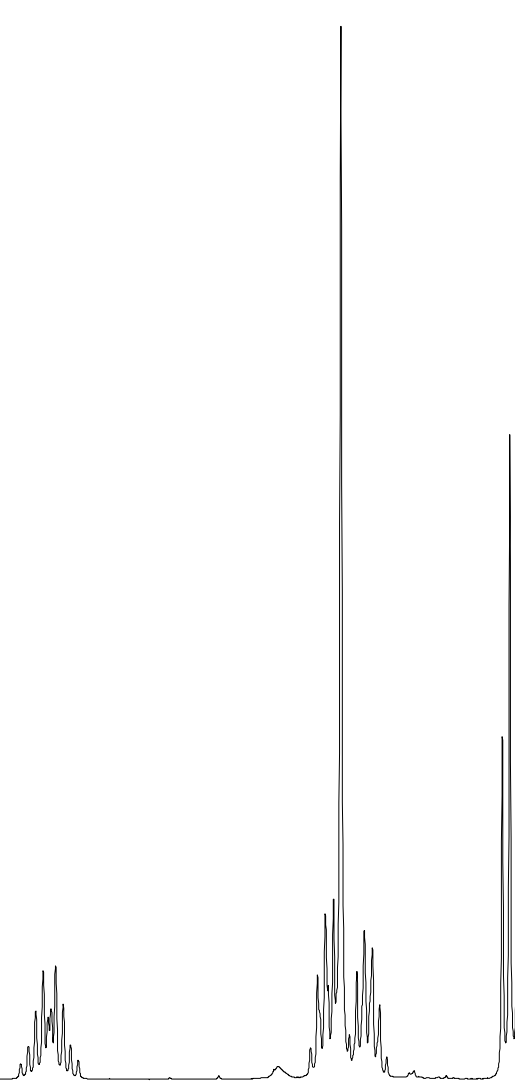




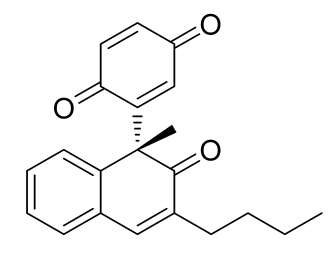

4d
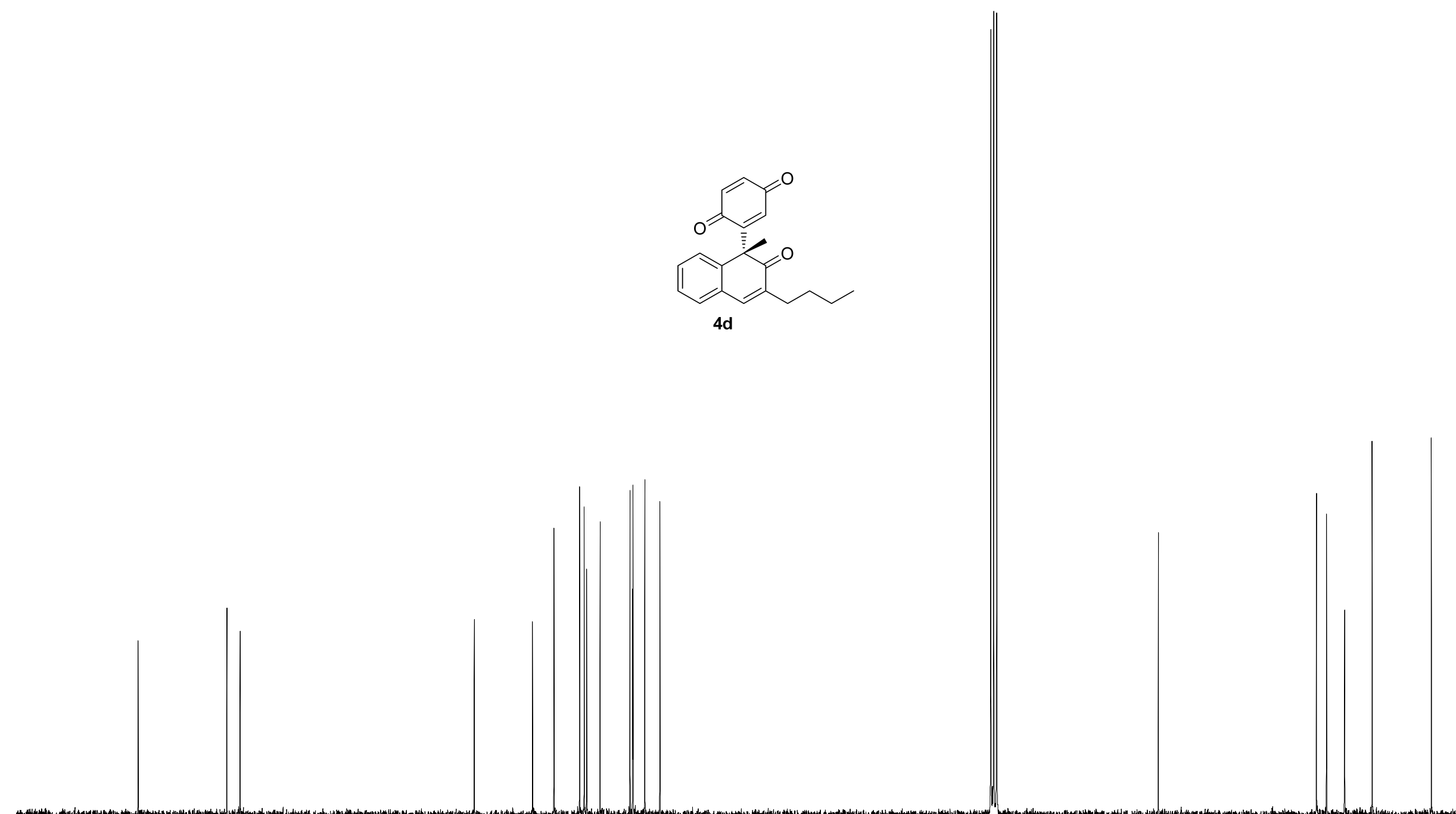

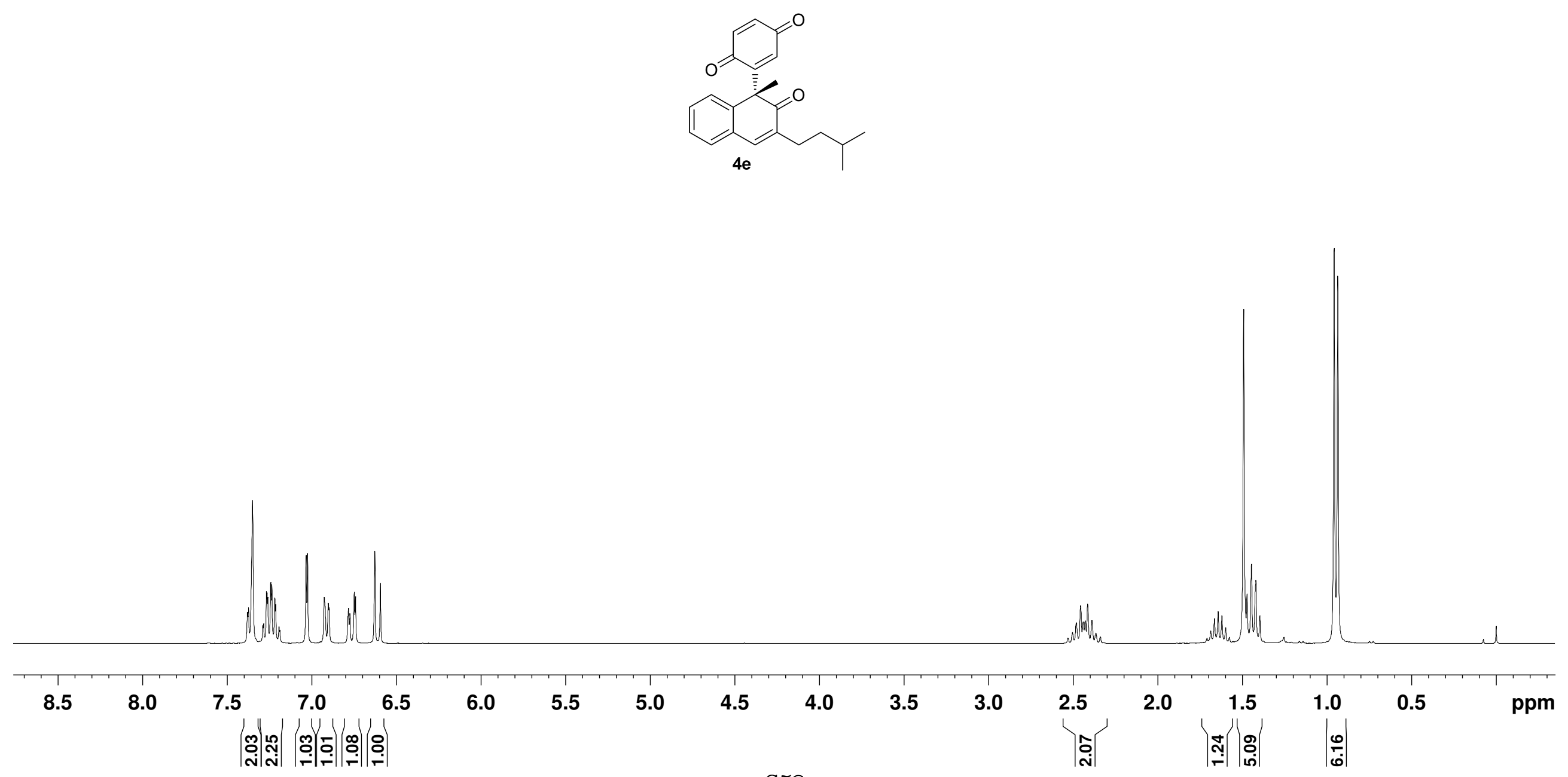

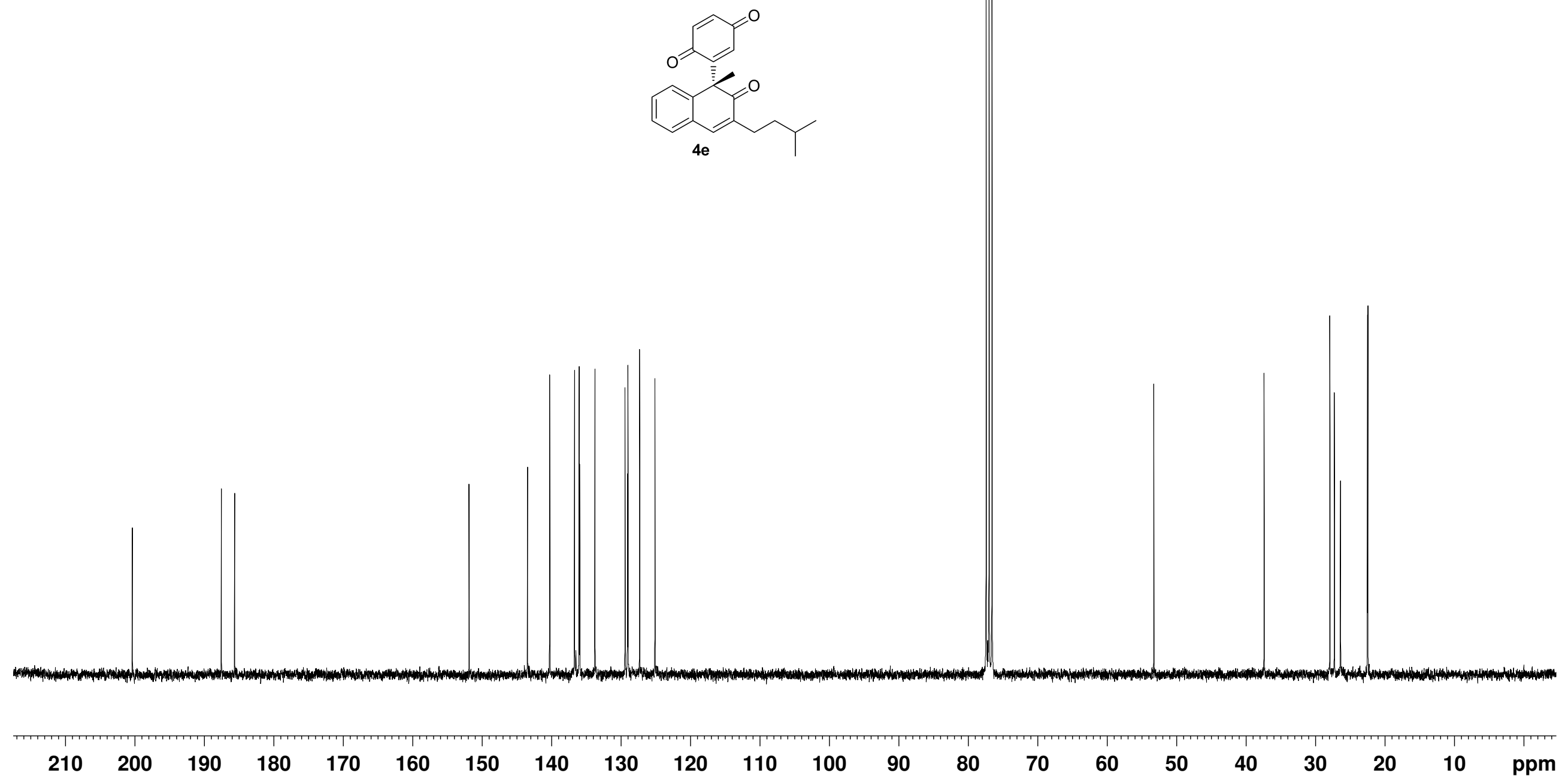


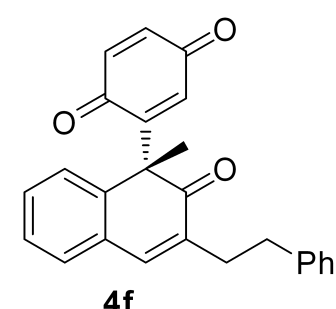

$4 f$

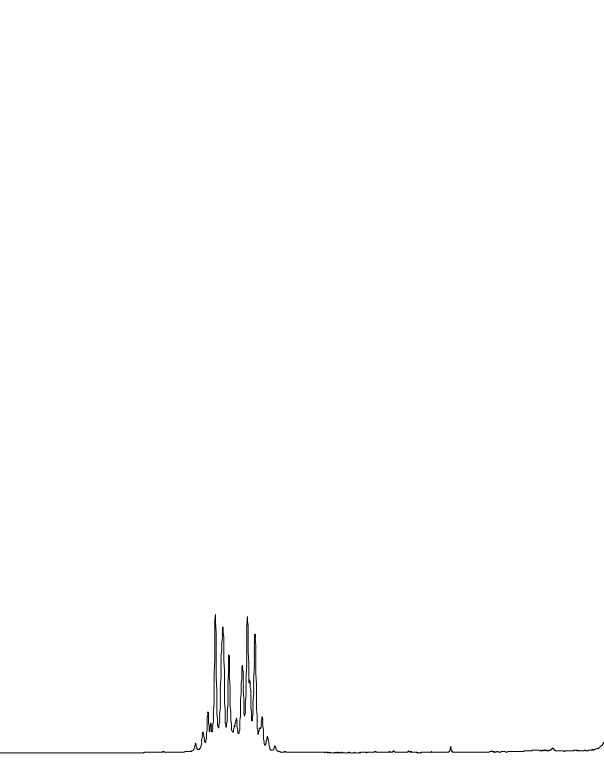

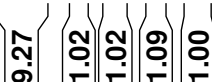

5

$2.0 \quad 1.5$ $\left|\begin{array}{c}\text { ְై } \\ \text { | }\end{array}\right|$ 


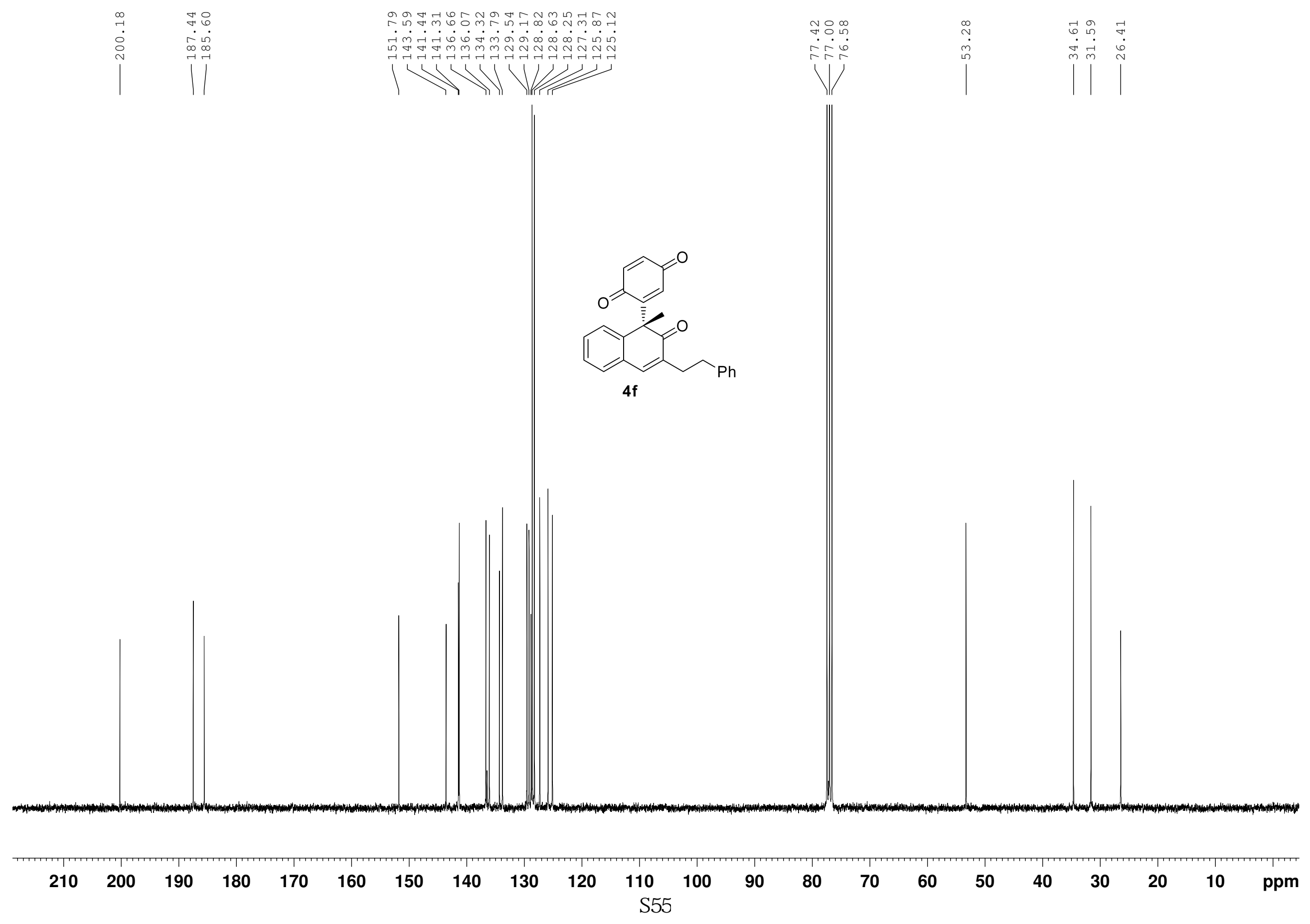




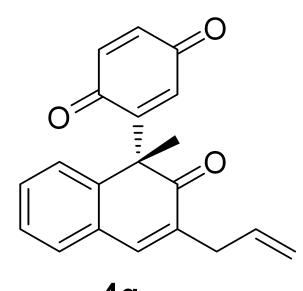

$4 \mathrm{~g}$

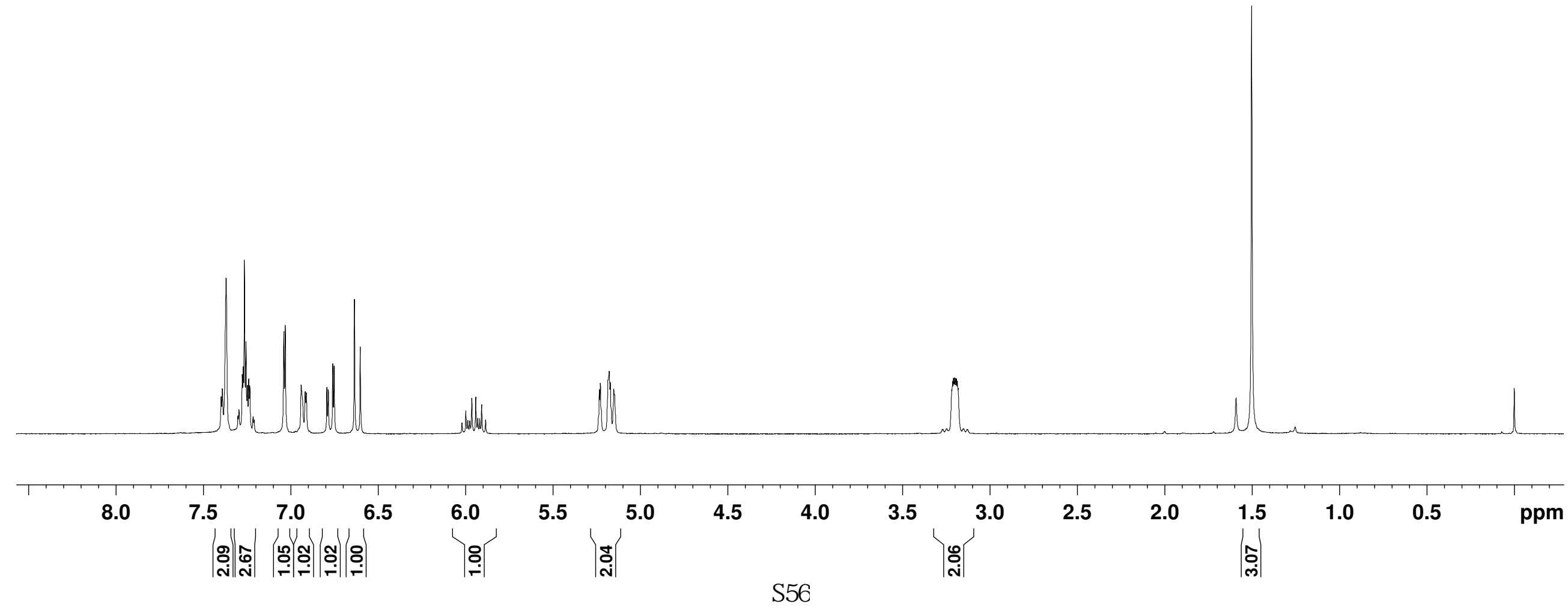



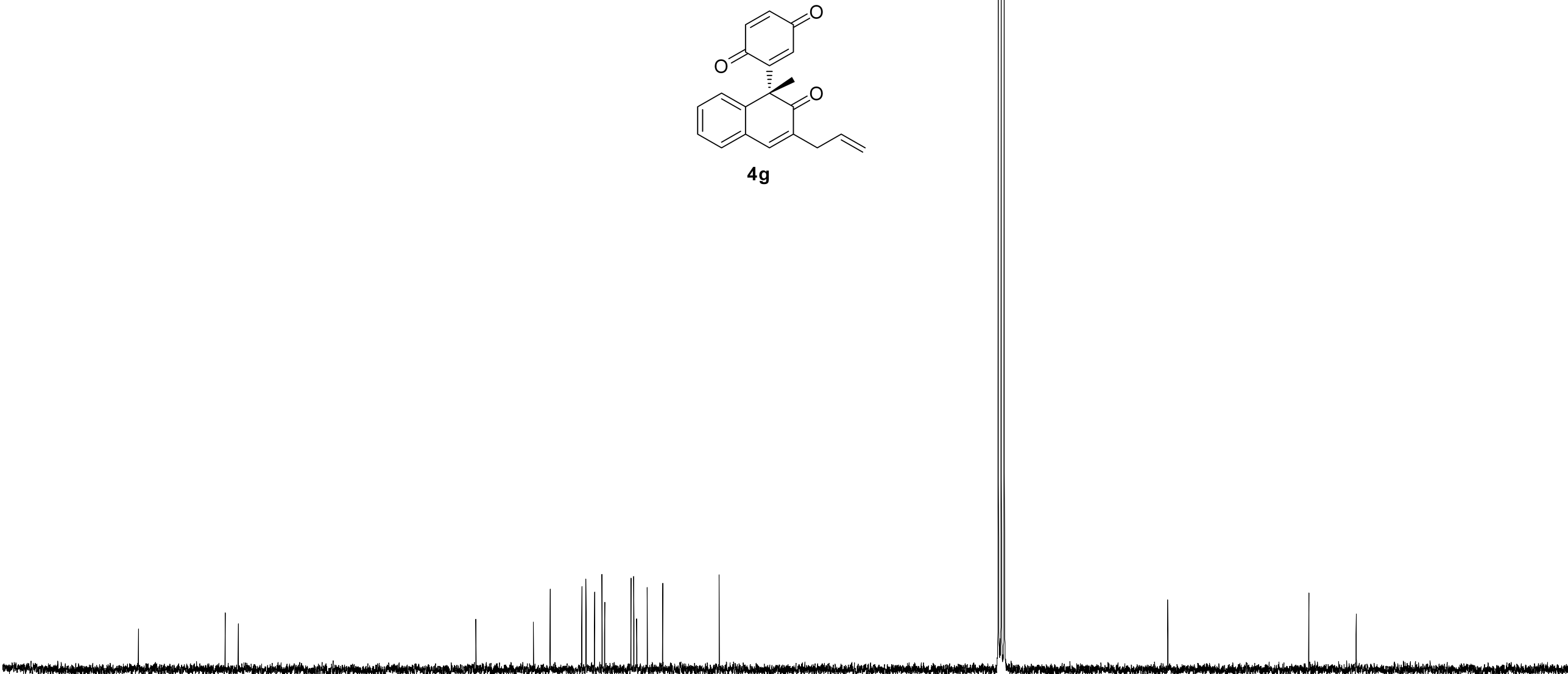

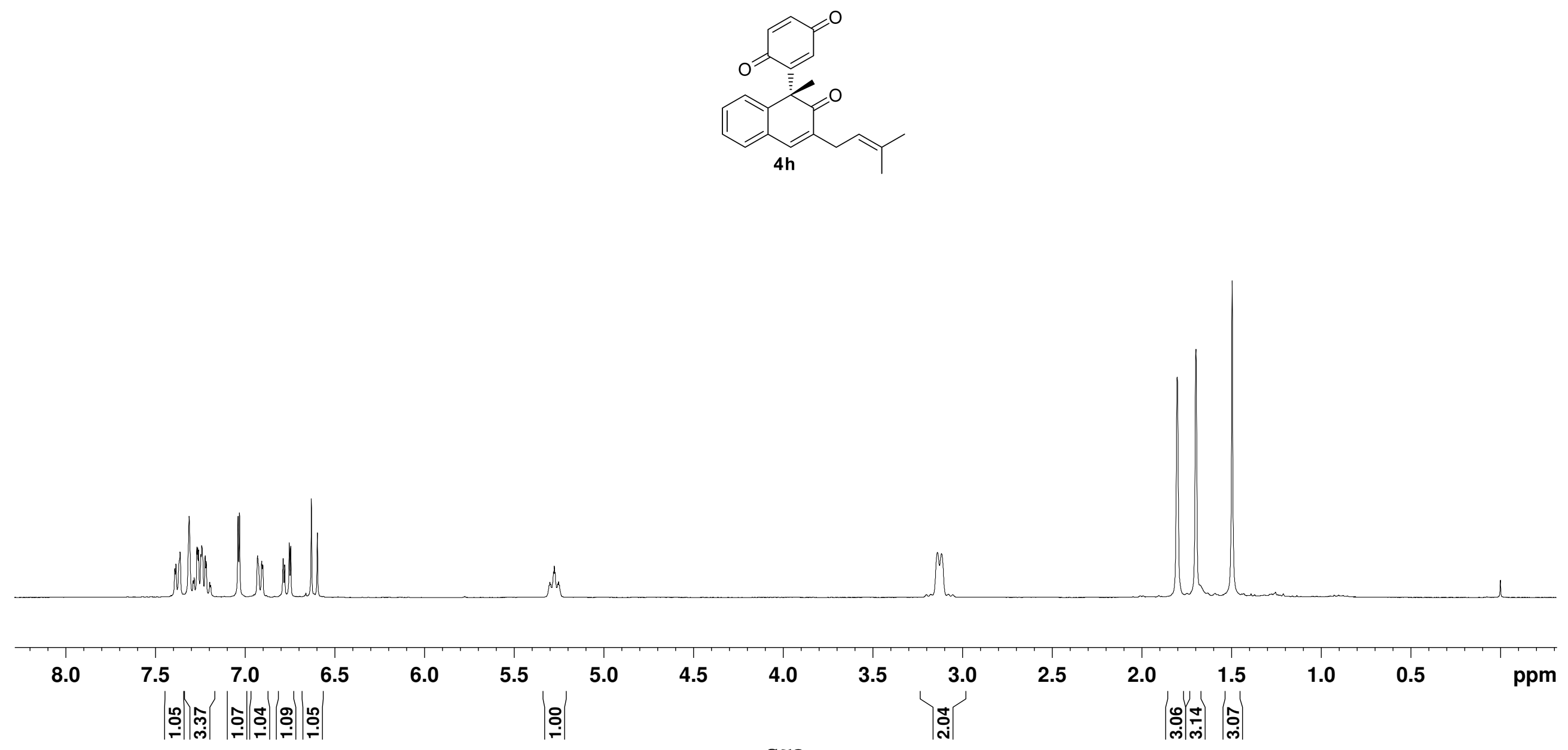

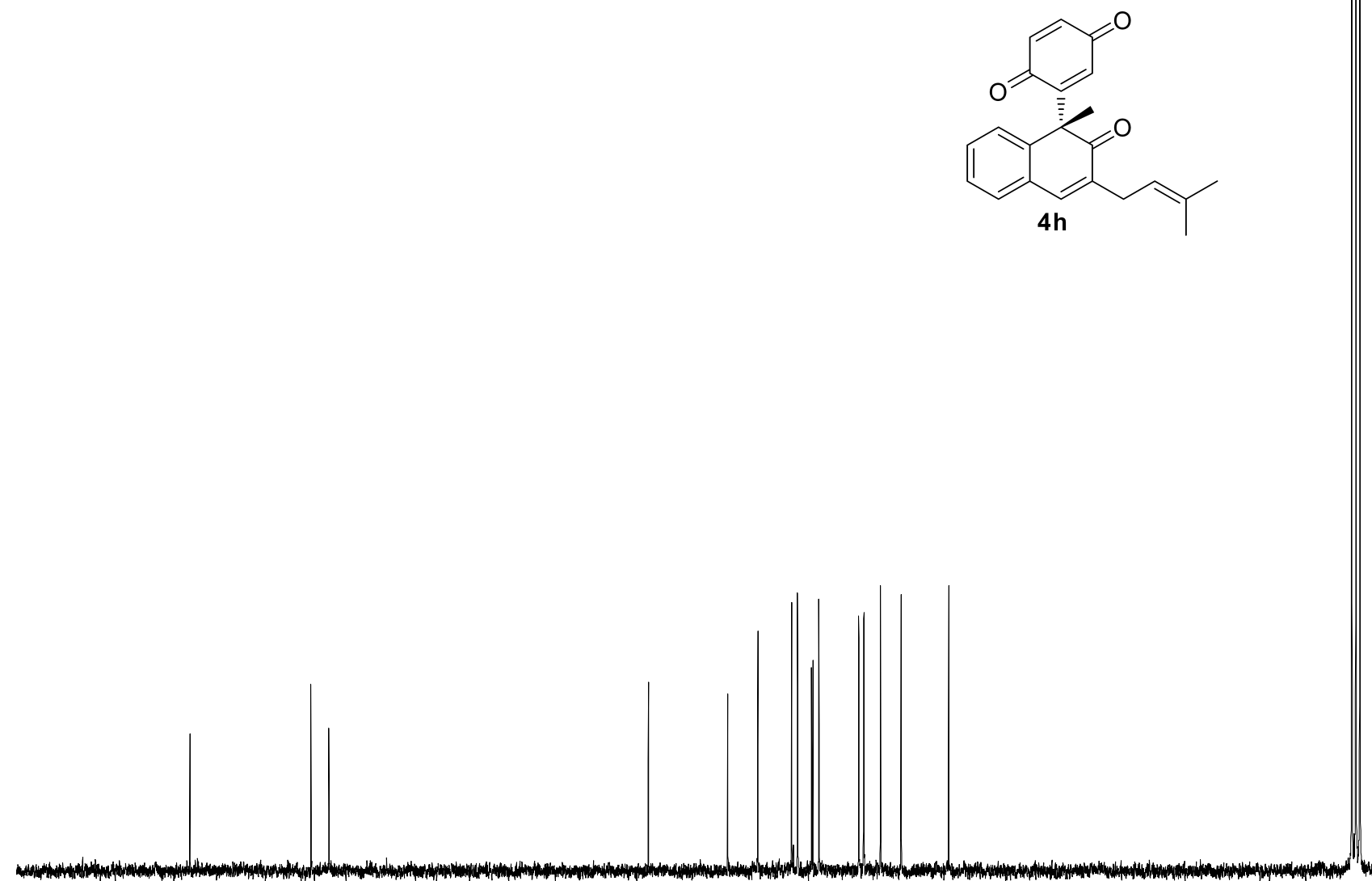

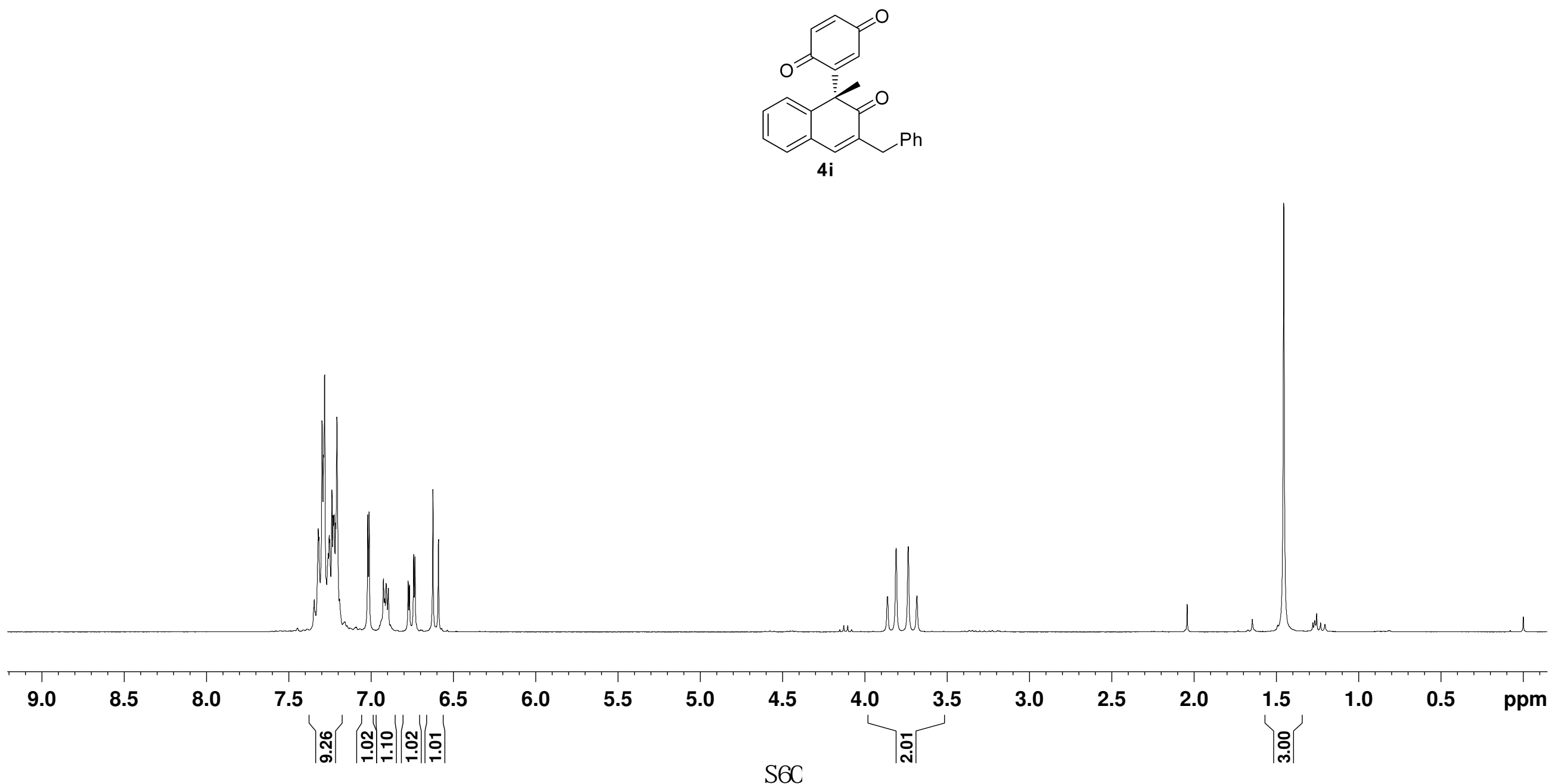

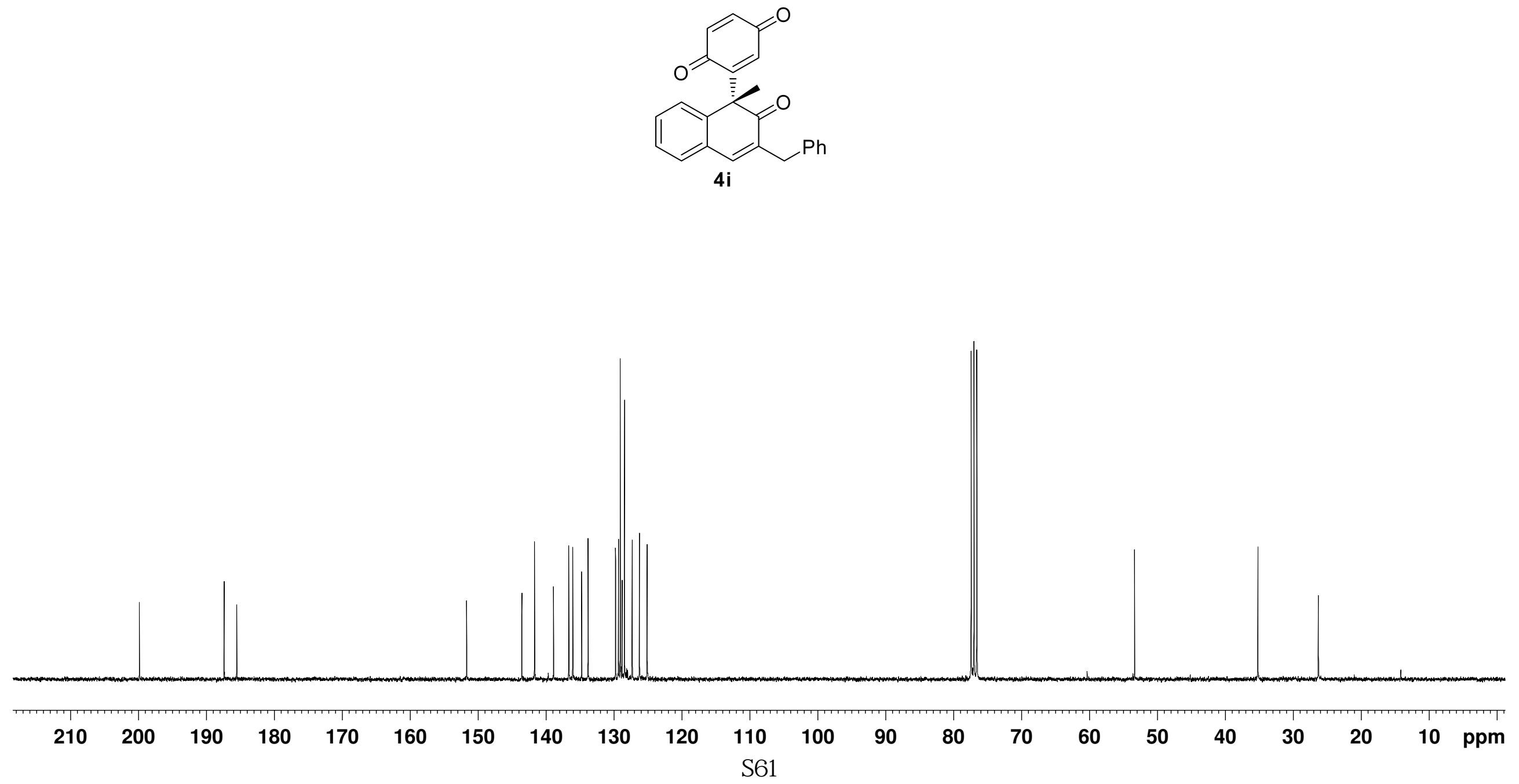


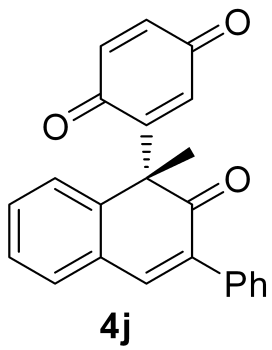

4j

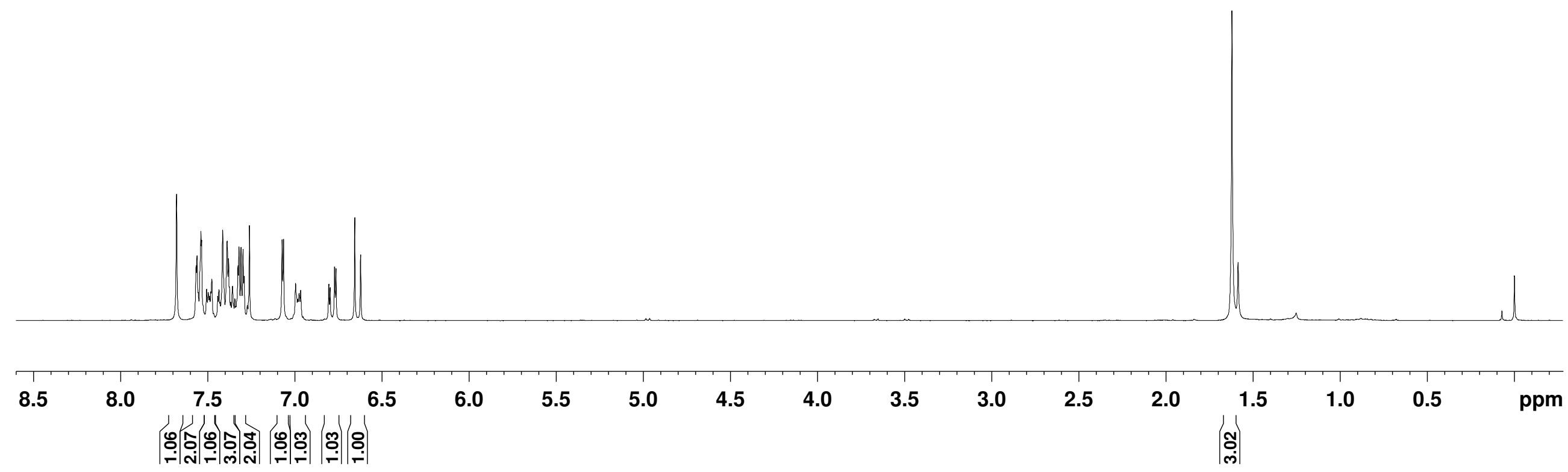



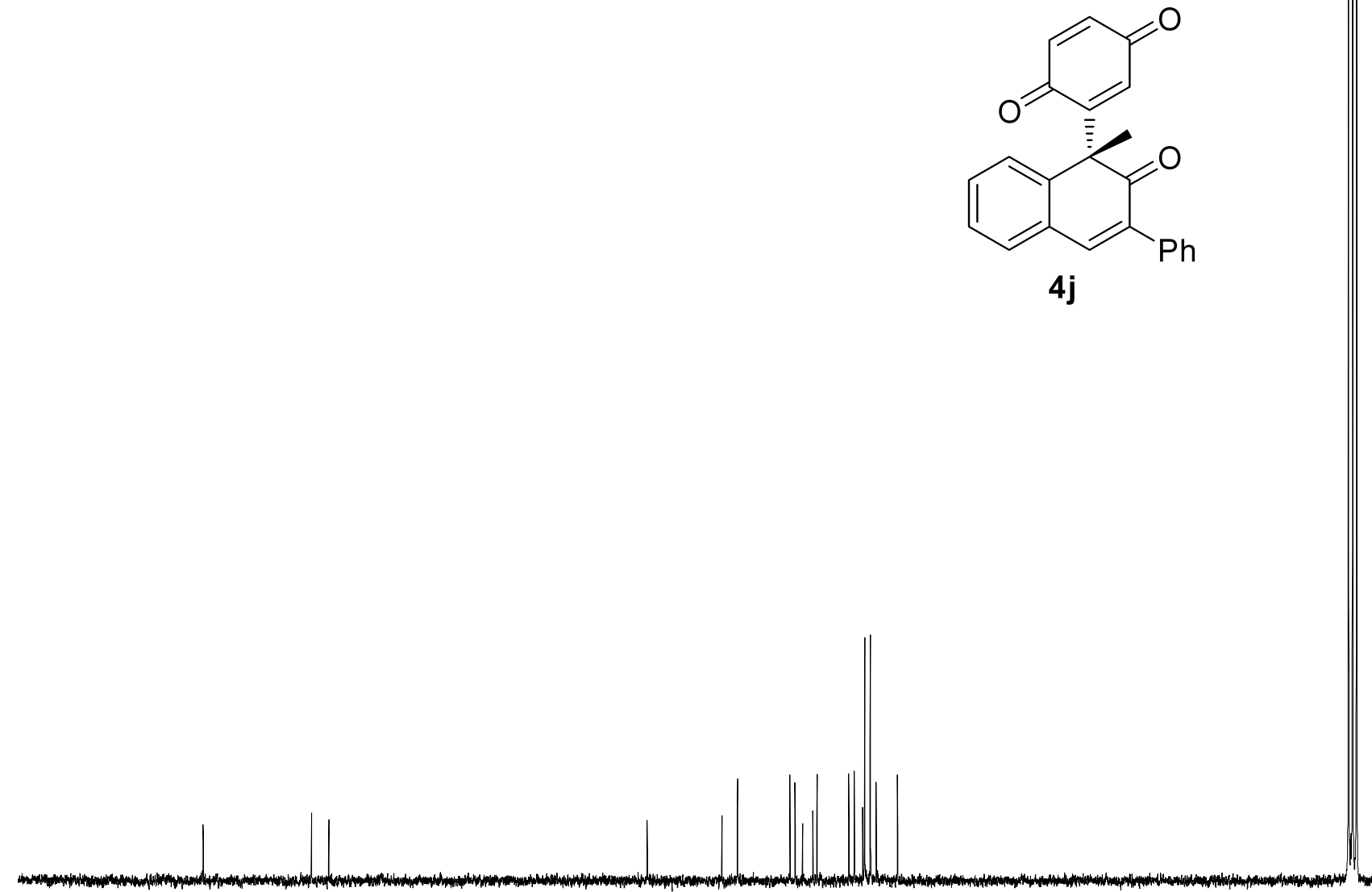


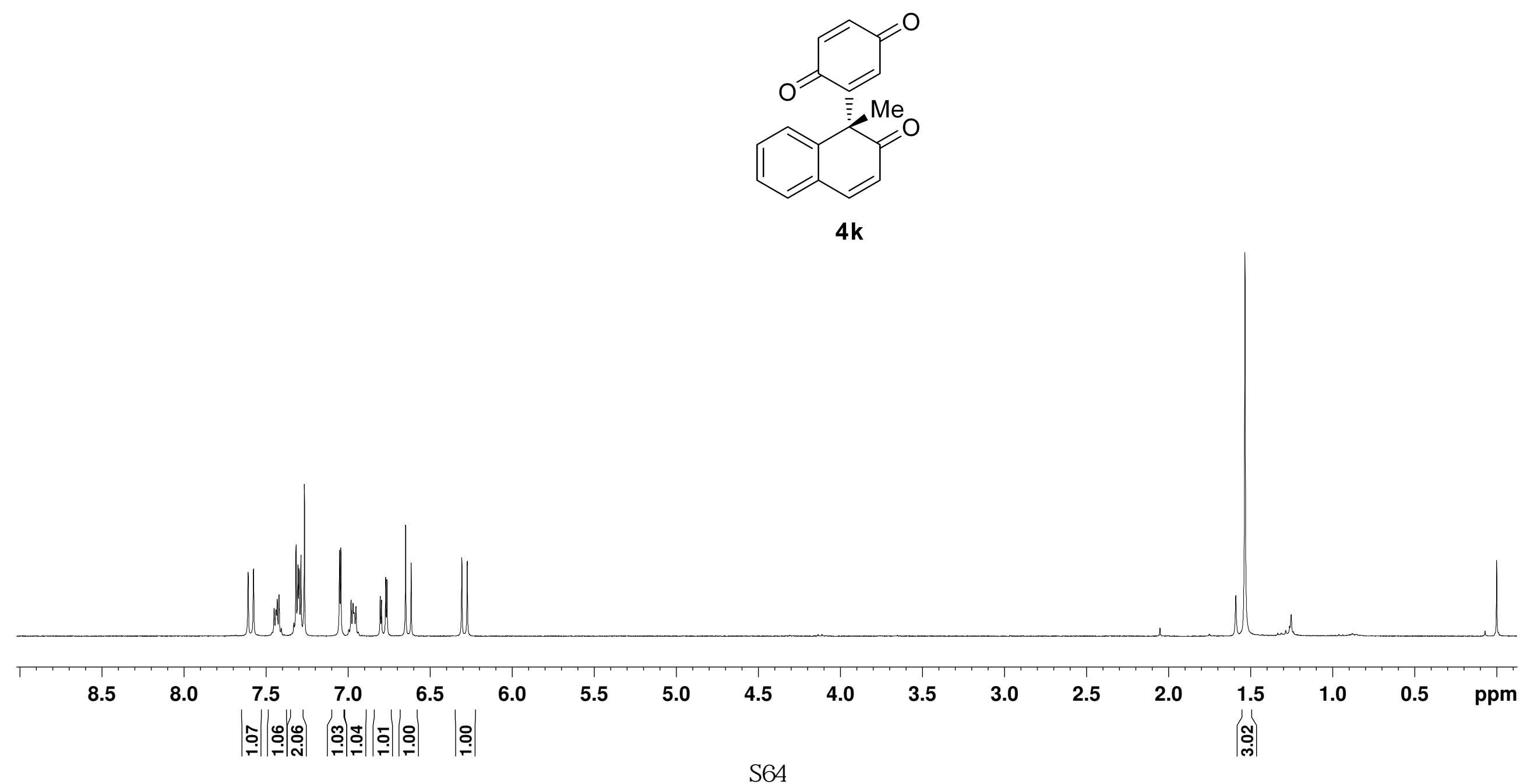




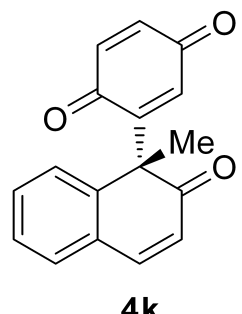

$4 \mathrm{k}$ 


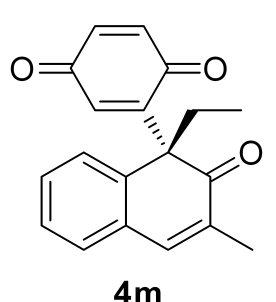

$4 \mathrm{~m}$

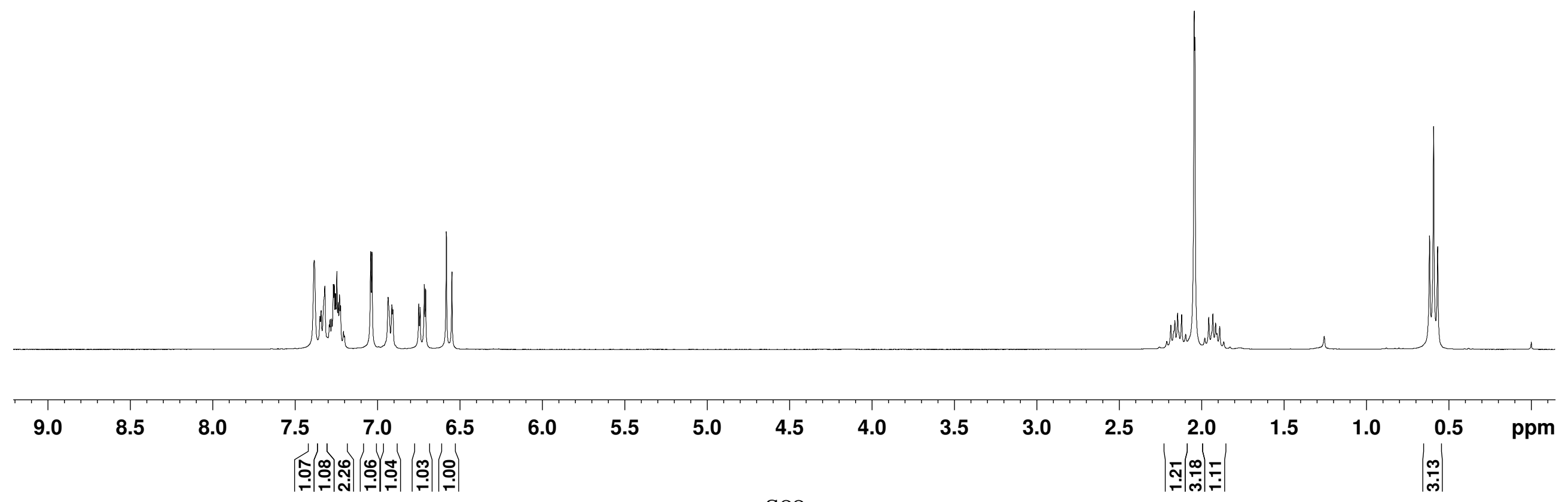




\section{군}

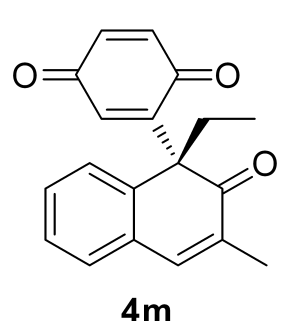

$4 \mathrm{~m}$

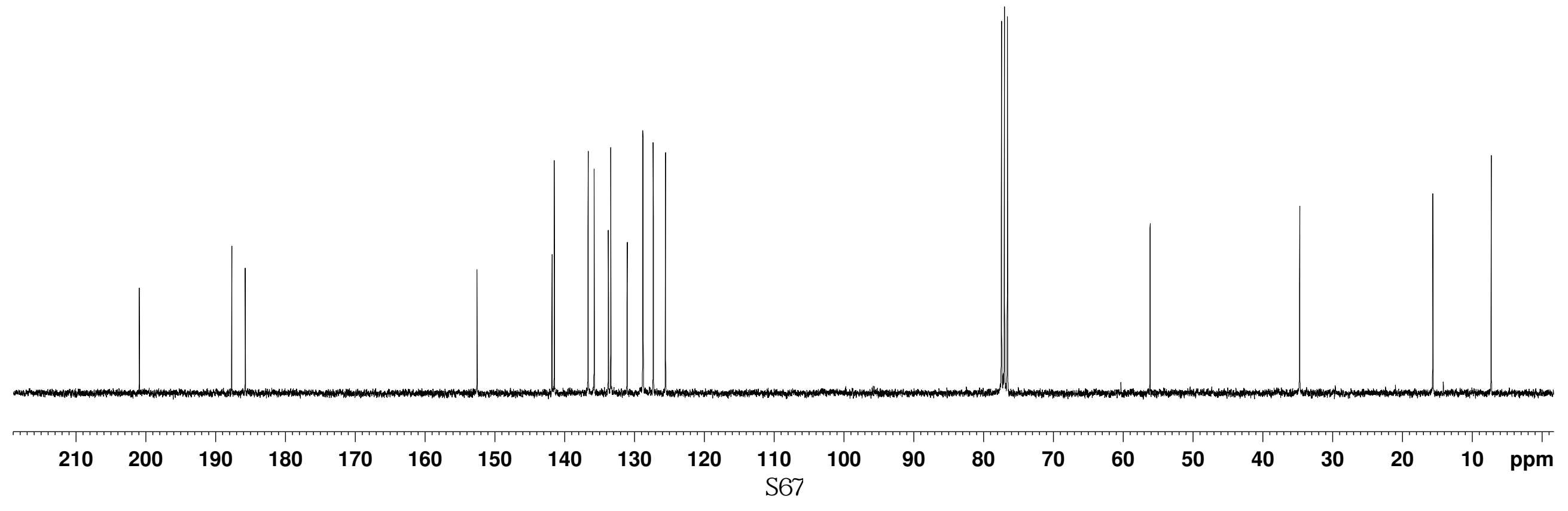




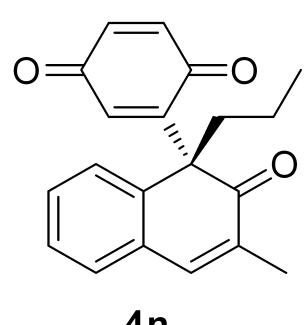

$4 n$

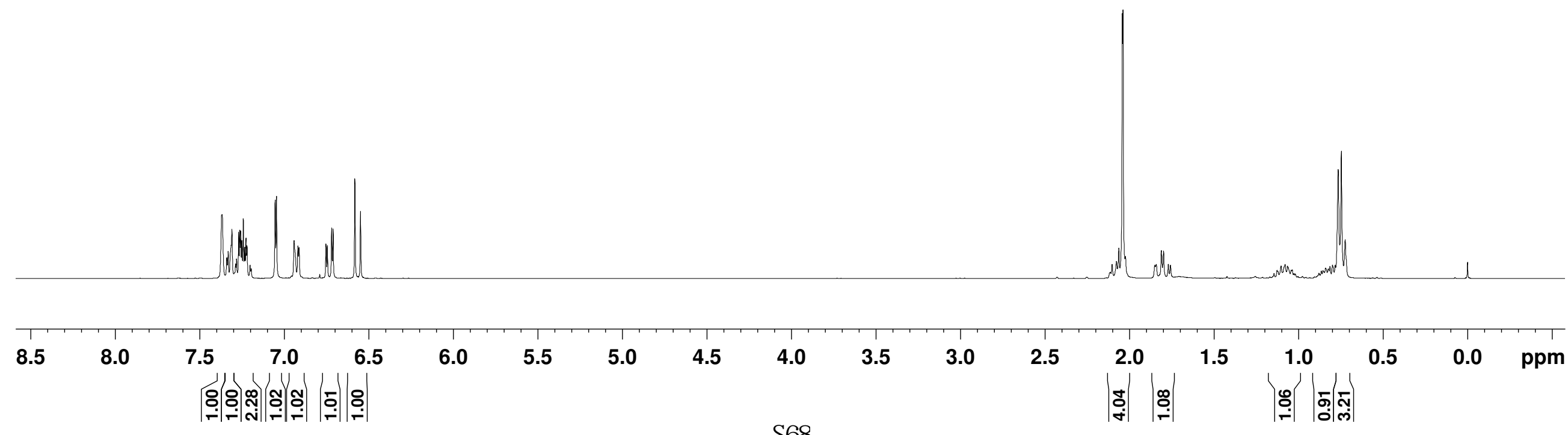




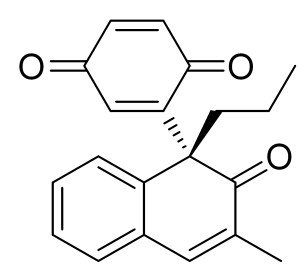

$4 n$

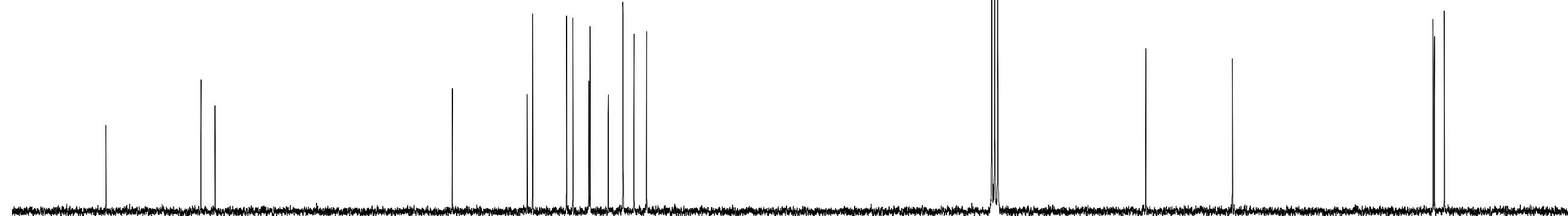

210200 

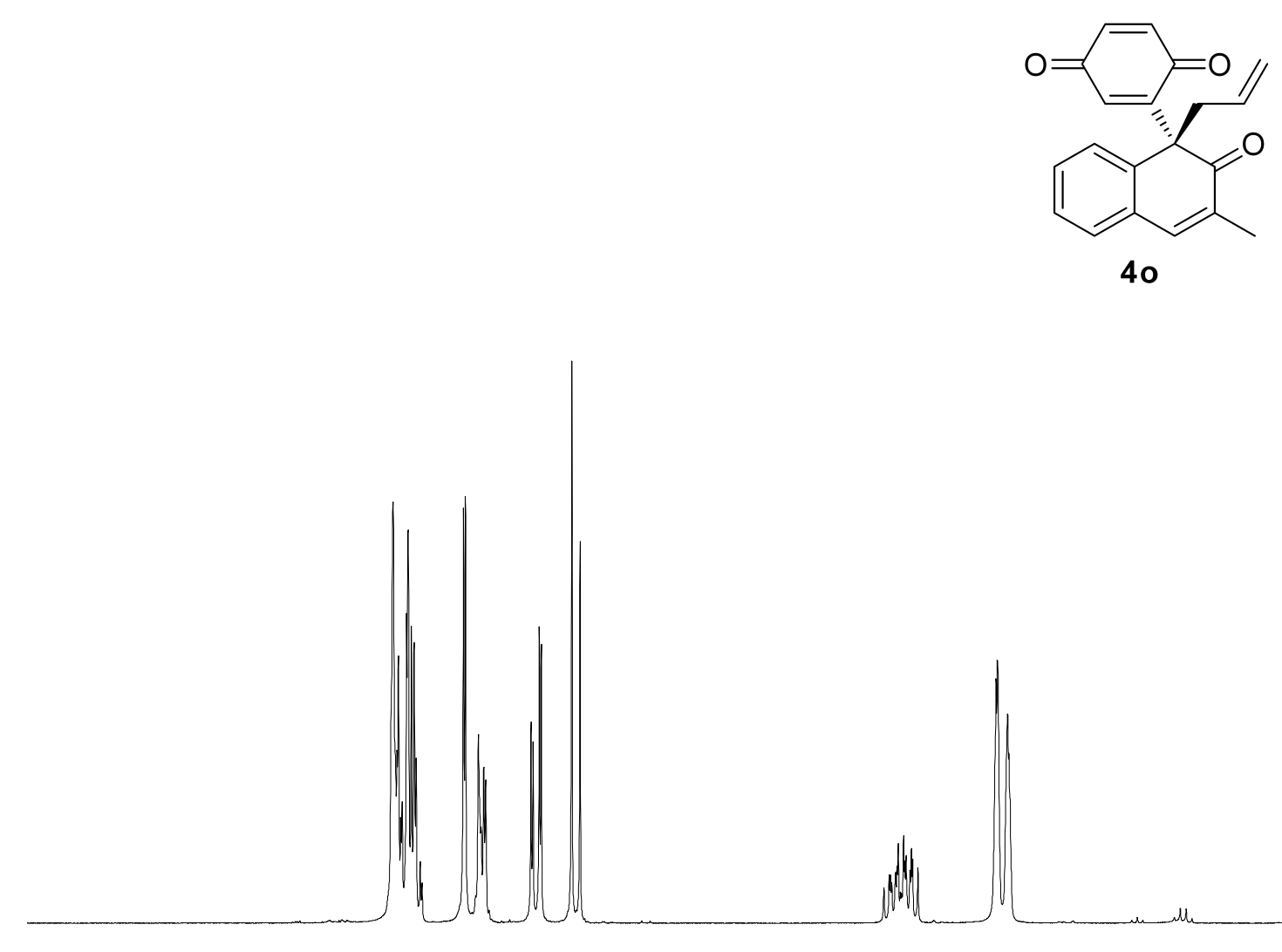

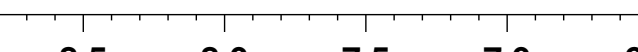

5.0 (8)

4.5 ำ 


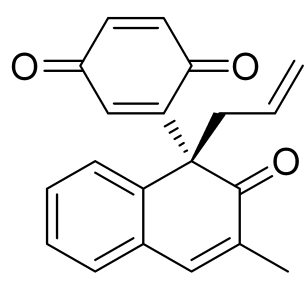

40 

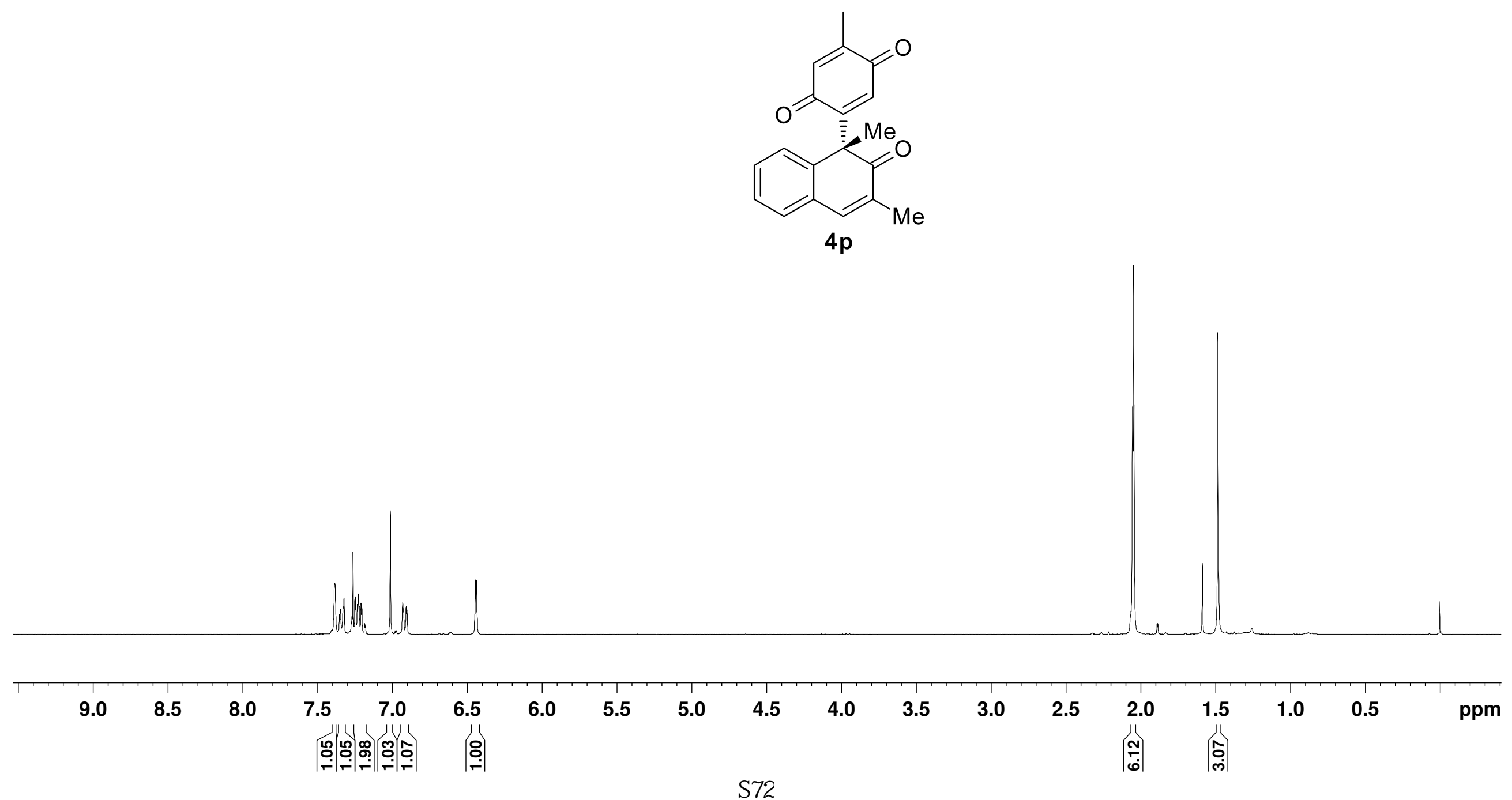


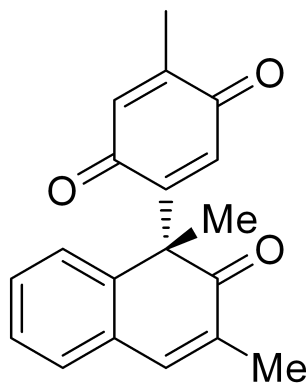




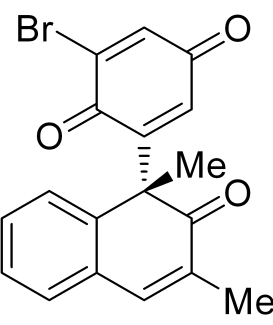

$4 q$

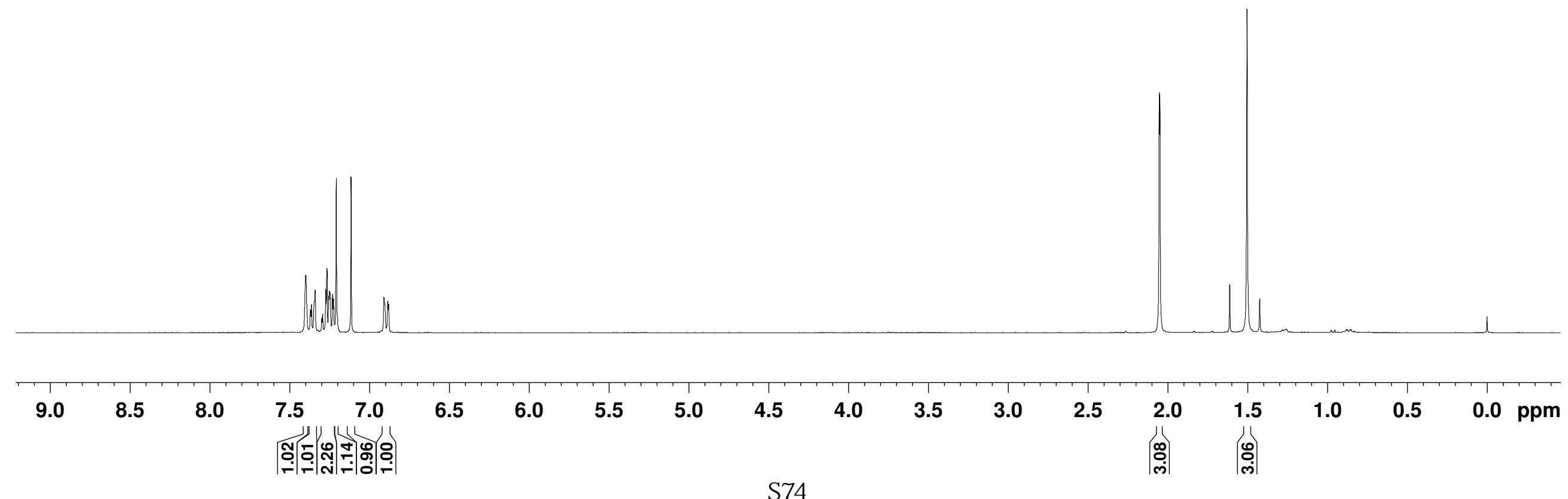




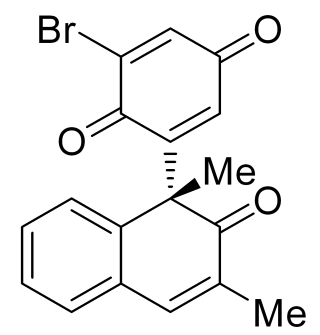

$4 q$
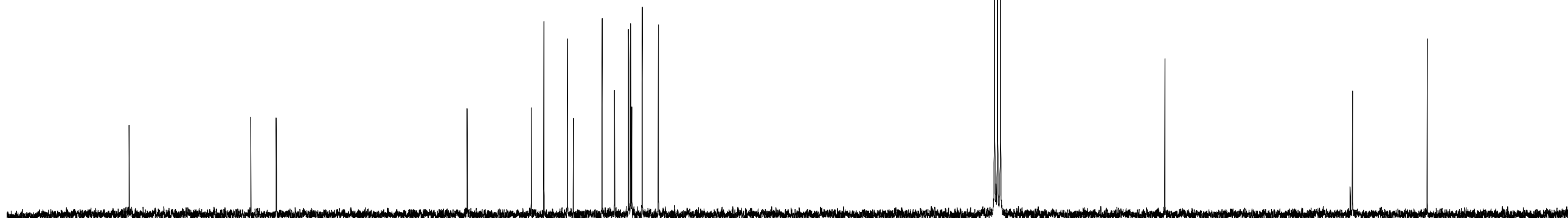

$\begin{array}{llllllllllllllllllllll}210 & 200 & 190 & 180 & 170 & 160 & 150 & 140 & 130 & 120 & 110 & 100 & 90 & 80 & 70 & 60 & 50 & 40 & 30 & 20 & 10 & \text { ppm }\end{array}$ S75 

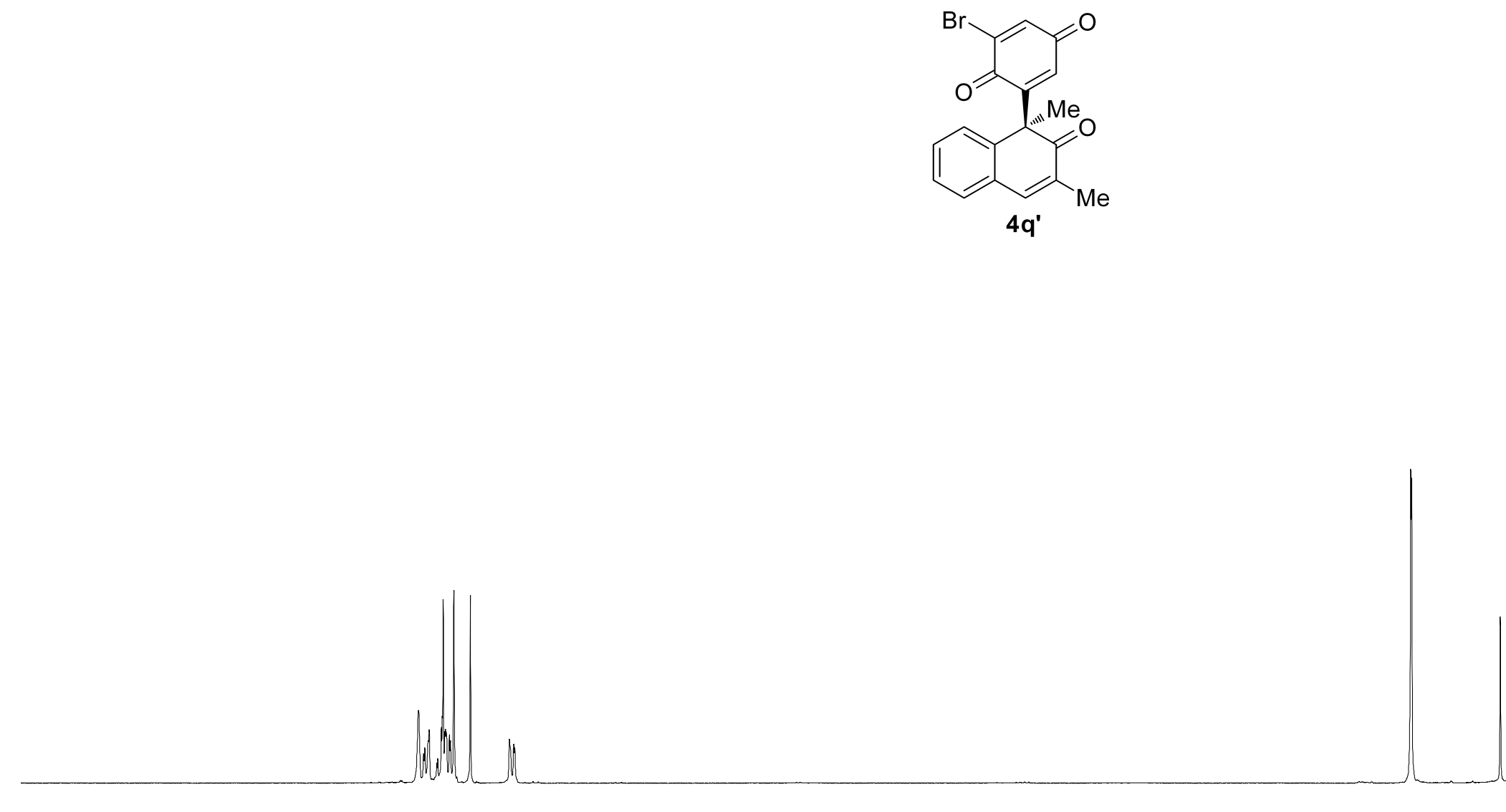

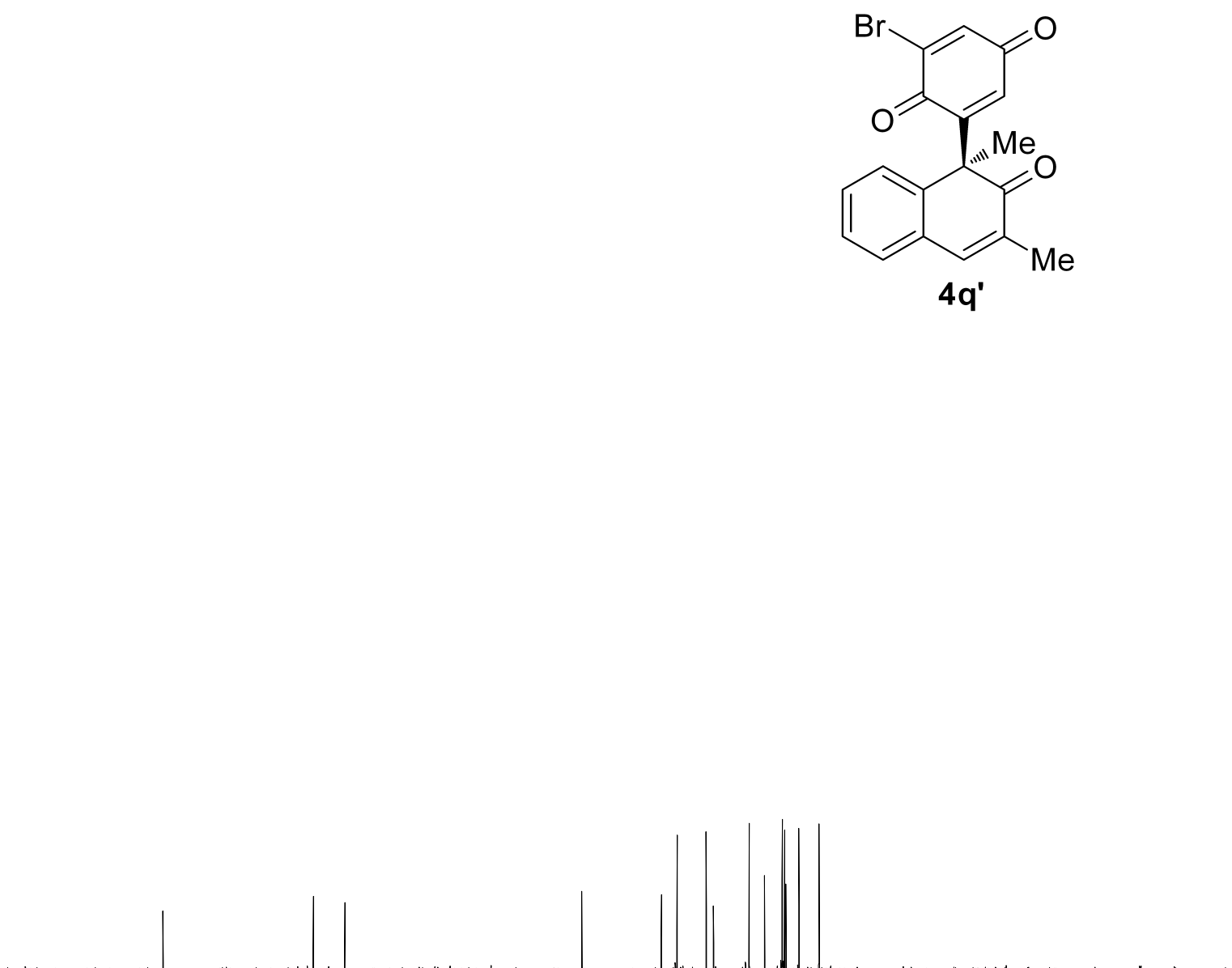

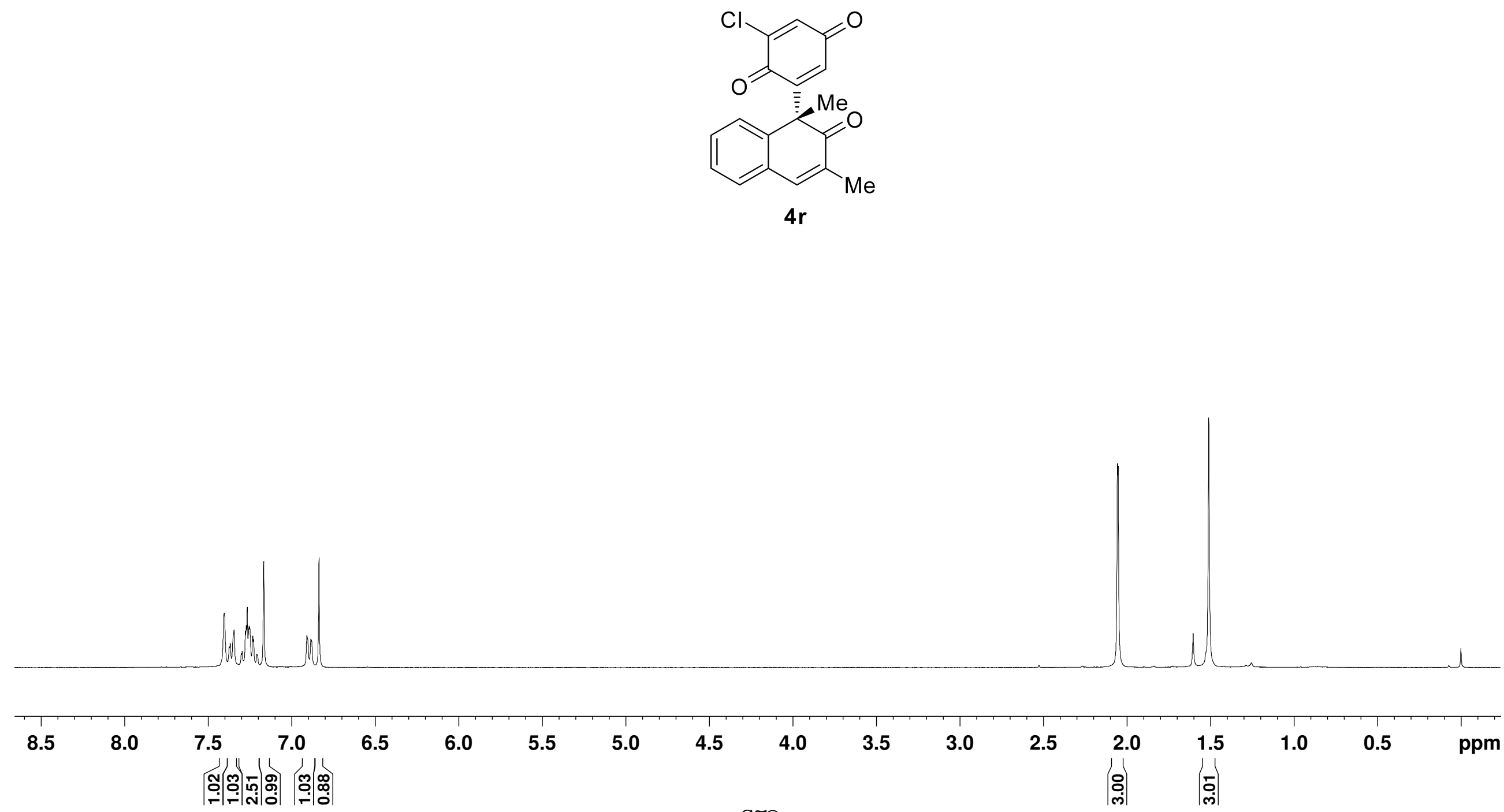


|
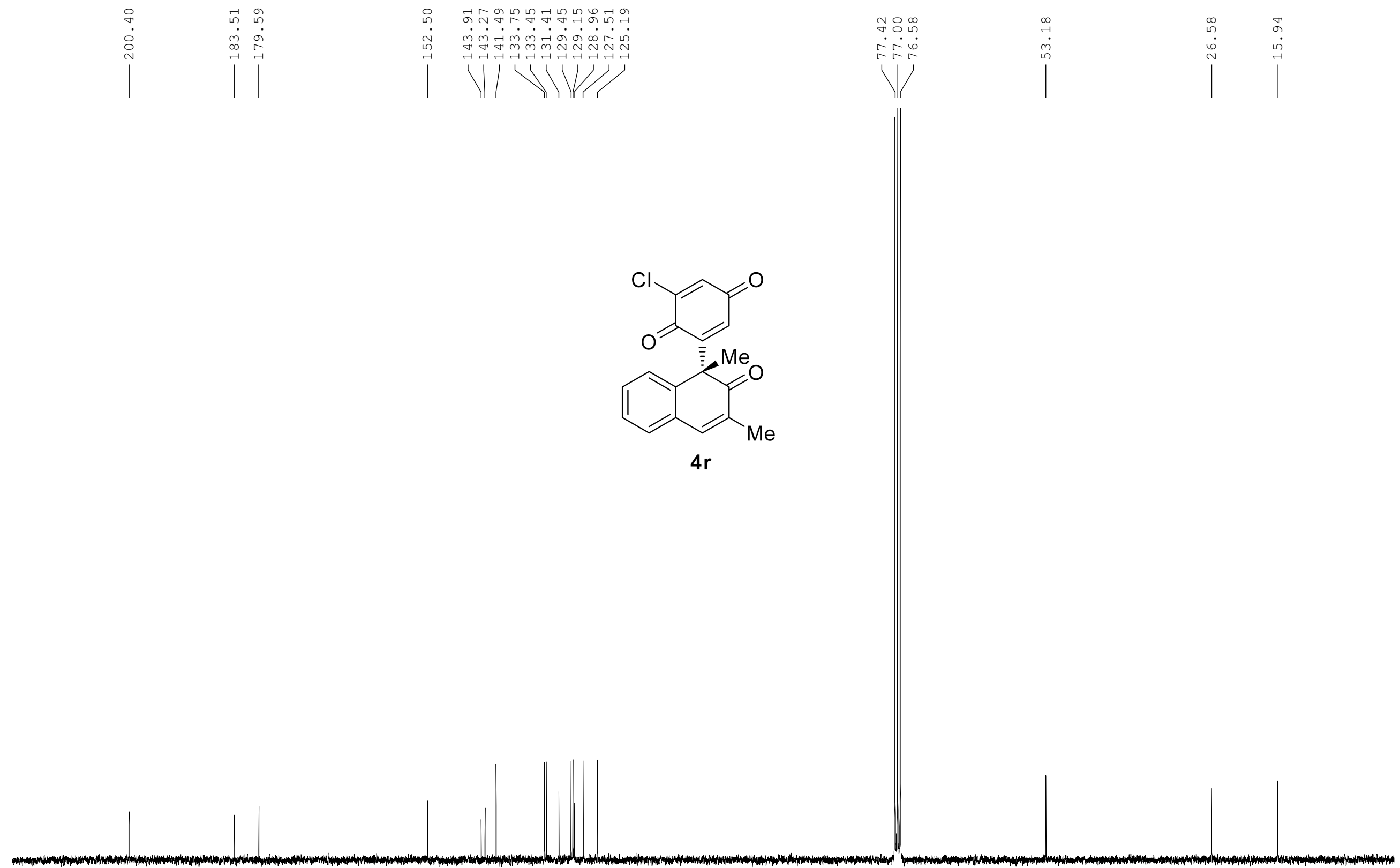


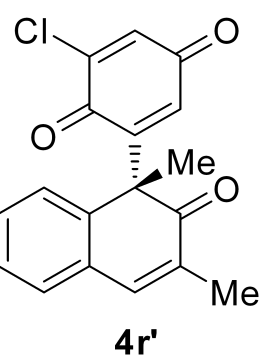

$\begin{array}{lllll}9.0 & 8.5 & 8.0 & 7.5\end{array}$

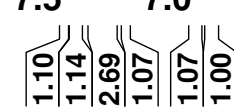

5.5

5.0

4.5

4.0

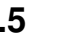
ลงกั 


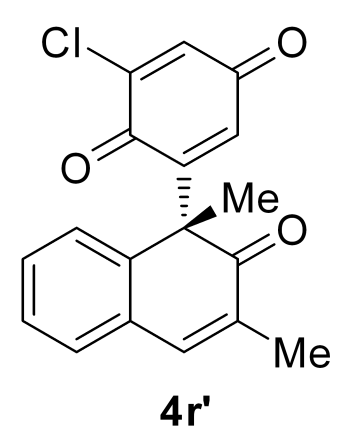



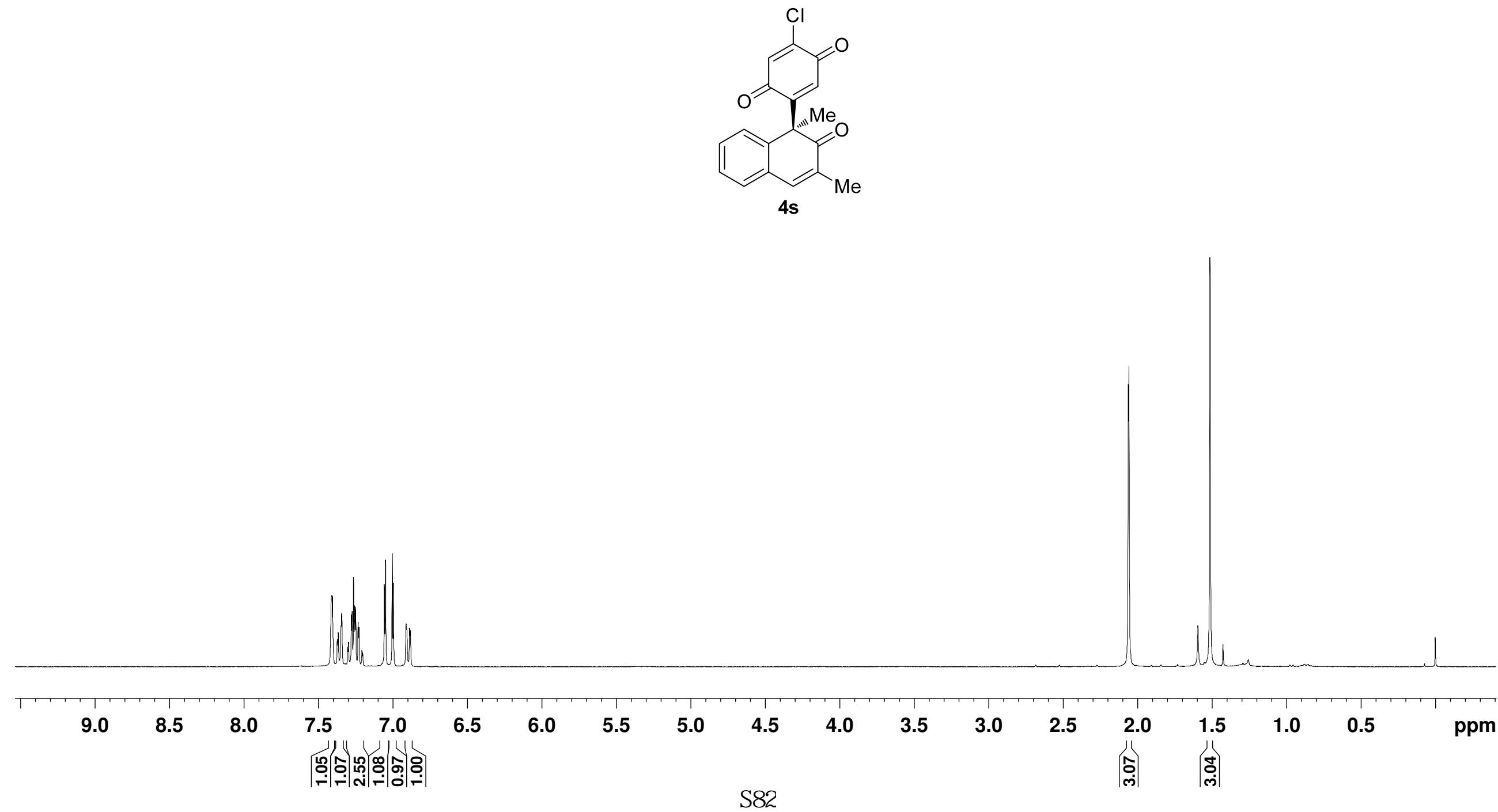

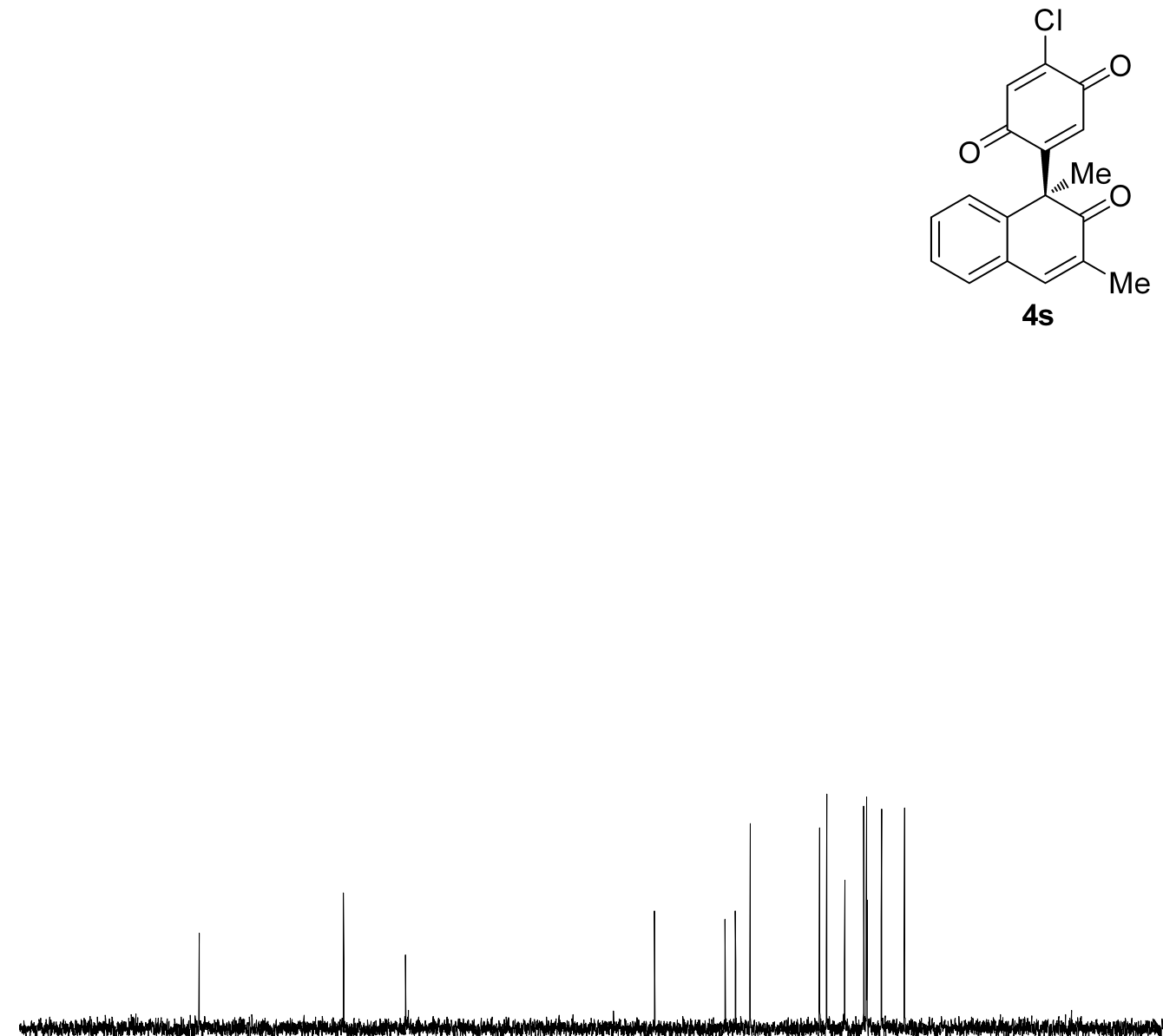

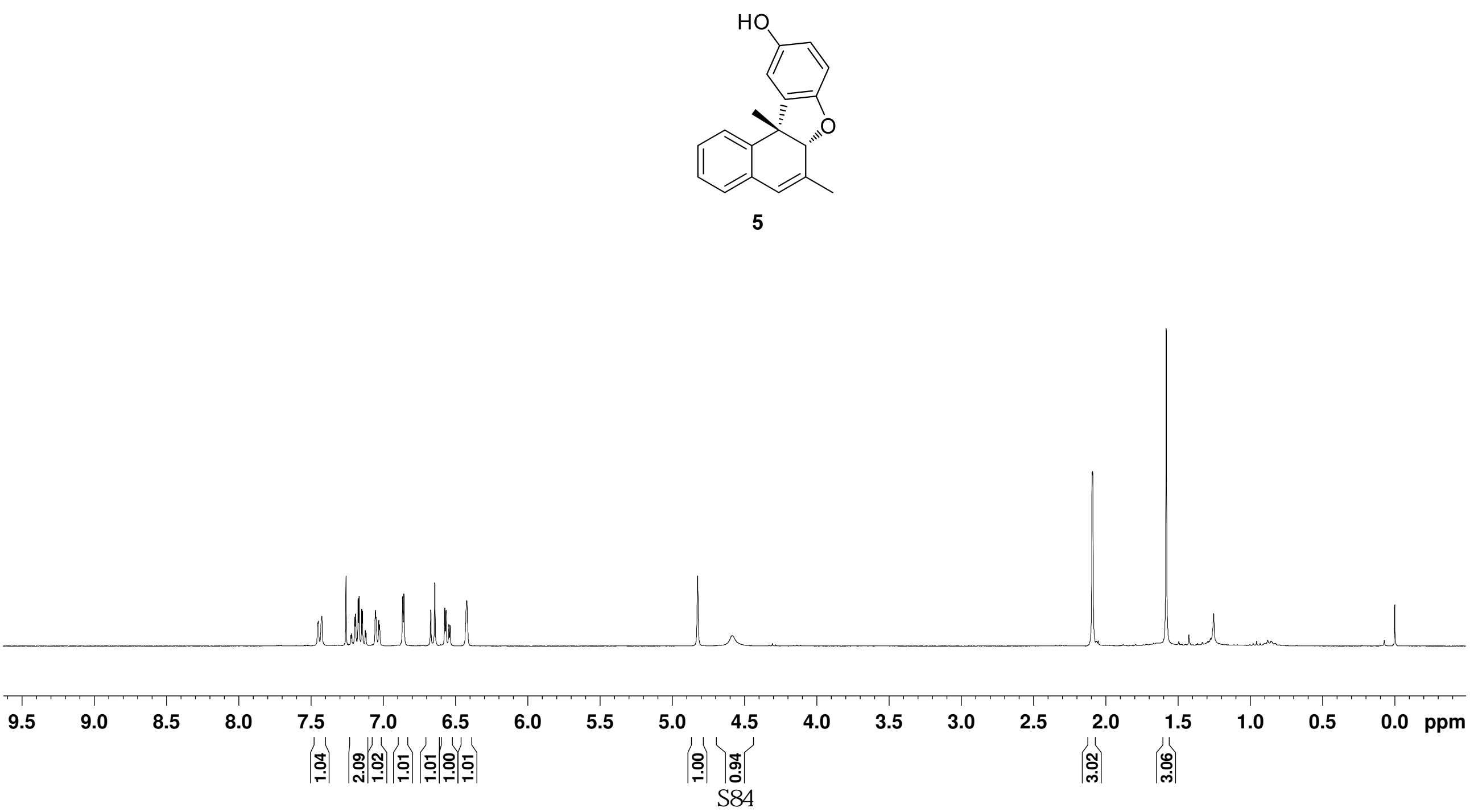


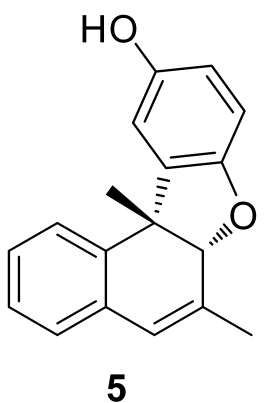

5 